Florida International University FIU Digital Commons

$11-5-2015$

\title{
A Mixed-Methods Examination among Young Adult Racially/Ethnically Diverse Bariatric Surgery Patients
}

Christine E. Spadola

Florida International University, cspad001@fiu.edu

DOI: $10.25148 /$ etd.FIDC000167

Follow this and additional works at: https://digitalcommons.fiu.edu/etd

Part of the Counseling Commons, Psychology Commons, and the Social Work Commons

\section{Recommended Citation}

Spadola, Christine E., "A Mixed-Methods Examination among Young Adult Racially/Ethnically Diverse Bariatric Surgery Patients" (2015). FIU Electronic Theses and Dissertations. 2271.

https://digitalcommons.fiu.edu/etd/2271 


\title{
FLORIDA INTERNATIONAL UNIVERSITY
}

Miami, Florida

\begin{abstract}
A MIXED-METHODS EXAMINATION OF ALCOHOL USE AMONG YOUNG
ADULT RACIALLY/ETHNICALLY DIVERSE BARIATRIC SURGERY PATIENTS
\end{abstract}

A dissertation submitted in partial fulfillment of

the requirements for the degree of

DOCTOR OF PHILOSOPHY

in

SOCIAL WELFARE

by

Christine E. Spadola

2015 
To: Interim Dean Mark L. Williams

R.Stempel College of Public Health and Social Work

This dissertation, written by Christine E. Spadola, and entitled A Mixed-Methods Examination of Alcohol Use among Young Adult Racially/Ethnically Diverse Bariatric Surgery Patients, having been approved in respect to style and intellectual content, is referred to you for judgment.

We have read this dissertation and recommend that it be approved.

$\begin{array}{r}\hline \text { Miriam Potocky } \\ \hline \text { Nicole Ruggiano } \\ \hline \text { Mark B. Padilla } \\ \hline \text { Sarah E. Messiah } \\ \hline \text { Eric F. Wagner, Major Professor }\end{array}$

Date of Defense: November 5, 2015

The dissertation of Christine E. Spadola is approved.

Interim Dean Mark L. Williams R.Stempel College of Public Health and Social Work

Dean Lakshmi N. Reddi University Graduate School

Florida International University, 2015 
(C) Copyright 2015 by Christine E. Spadola

\begin{abstract}
All rights reserved.
Copyright permission (license number 3716151144398) was obtained from Wiley publishers to reproduce parts of the following publication: Spadola, C. E., Wagner, E. F., Dillon, F. R., Trepka, M. J., La Cruz-Munoz, D., \& Messiah, S. E. (2015). “Alcohol and Drug Use Among Postoperative Bariatric Patients: A Systematic Review of the Emerging Research and Its Implications.” Alcoholism: Clinical and Experimental Research, 39 (9), 1582-1601.
\end{abstract}




\section{DEDICATION}

This dissertation is dedicated to my parents. Mom and Dad, thank you for your love and humor and raising me to work hard, read, recycle, and check my tire pressure monthly. 


\section{ACKNOWLEDGMENTS}

I would like to thank my major professor and one of the most talented writers I know, Eric Wagner, for his guidance, wisdom, and wit throughout the doctoral program. Thank you for always looking out for my best interests, as well as providing the best restaurant recommendations in cities that host academic conferences!

Sarah Messiah, it was a conversation that we had a few years back, in your office that solidified my decision to return to school. Your strength and work ethic continue to inspire me. Thank you for supporting me, encouraging me, and believing in me from day one.

Thank you to the rest of my esteemed dissertation committee, Miriam Potocky, Nicole Ruggiano, and Mark Padilla, for your genuine interest in my research and always providing helpful feedback.

I am grateful to David Saltman, who first told me about the PhD program and over several lunches together fueled with philosophical discussions, convinced me to apply. I look forward to many more philosophical lunches with you as well as conversations about New Jersey, of course!

I am also grateful to Rich Beaulaurier whose qualitative research class further inspired me to take the doctoral degree plunge, and who remained supportive and encouraging throughout the doctoral program.

Thank you to Mario De La Rosa, Mary Jo Trepka, and Frank Dillon. I learned a lot from each of you through my C-SALUD/CRUSADA fellowship. I am also grateful 
that through the fellowship, I was able to meet and be mentored by a wonderful bariatric psychologist, Melodie Moorehead.

I am very thankful for a handful of other faculty, researchers, and clinicians including Mary Helen Hayden, Peggy Rios of the Cancer Support Community of Greater Miami, Bryan Page, Marion C. Kiley, Leah Varga, Alice Cepeda, Avelardo “Lalo” Valdez, and Gregory Hunt.

Thank you to my most supportive FIU-BRIDGE family. I am very lucky to work in such a warm and productive environment that is also filled with laughter and (very frequently) food!

I am eternally grateful to Dr. Michelle Hospital, who was training for her first full marathon in the Miami heat when I was in throes of my dissertation writing. Michelleyou have both inspired and supported me more than you will ever know.

To my PhD comrades- your support and encouragement will never be forgotten. Neither will our most necessary outings. I am very happy I got to take this journey with you all!

Very importantly, this research would not have been possible without the many research participants who gave their time and provided sensitive information so that practice, policy, and post-operative follow up care can hopefully be improved for future bariatric surgery patients.

Finally, I would like to thank Jaime Gow for always making me laugh, and knowing when to buy me chocolate. I'm very lucky to have you in my life. Andiamo! 


\begin{abstract}
OF THE DISSERTATION
A MIXED-METHODS EXAMINATION OF ALCOHOL USE AMONG YOUNG ADULT RACIALLY/ETHNICALLY DIVERSE BARIATRIC SURGERY PATIENTS

\author{
by
}

Christine E. Spadola

Florida International University, 2015

Miami, Florida

\title{
Professor Eric F. Wagner, Major Professor
}

Bariatric, or weight loss, surgery (WLS) is known as the most effective treatment for severe obesity, and the number of bariatric surgeries performed in the United States has more than tripled over the past two decades. Despite the potential health benefits of WLS (i.e., reversal of type 2 diabetes), research has revealed problematic alcohol use among WLS patients, in part associated with the following risk factors: the prevalence of a lifetime alcohol use disorder (AUD), the Roux-en-Y gastric bypass (RYGB) procedure, younger age, and an increased post-surgical sensitivity to alcohol. There is reason to believe both (a) young adult and (b) racial/ethnic minority bariatric surgery patients could be particularly prone to problematic drinking post-surgery, as both demographic groups represent an elevated risk of problematic alcohol use in the general population. Despite this, to date, there has been no in-depth assessment of alcohol use among young adult WLS patients. This dissertation utilized a sequential exploratory mixed methods design to examine alcohol use among young adult, racially/ethnically diverse WLS patients. Descriptive and inferential analyses examined alcohol use patterns and predictors of problematic alcohol use for 69 young adults who had WLS. Nearly one quarter of the 
sample $(24.6 \%, n=17)$ reported an increase in alcohol use after WLS. Age, time since surgery, the RYGB procedure, and having a pre-operative alcohol use disorder (AUD) when entered into the model together, were not a predictive model of frequency of alcohol use or problematic alcohol use after WLS (F [4, 64] $=2.3, p=.067)$. However, the diagnosis of a pre-operative AUD was associated with an increased frequency of alcohol use post WLS, holding all other predictors constant $(\mathrm{p}<.05)$. Following a purposive sampling approach, the subset that reported an increase in alcohol use was recruited for an in-depth, qualitative interview $(\mathrm{n}=12)$. Four major themes emerged concerning an increase in alcohol use after WLS: (1) alcohol as a substitute for food, (2) alcohol use sensitivity, (3) socialization, and (4) alcohol as a coping mechanism. By understanding factors and drinking motivations associated with alcohol use post-WLS, targeted pre and post-surgical counseling interventions can be developed to better educate and address problematic alcohol use among WLS patients. 


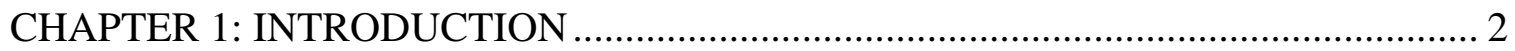

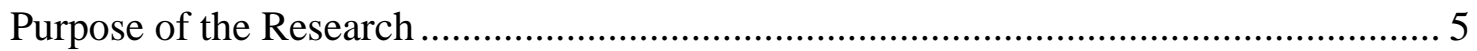

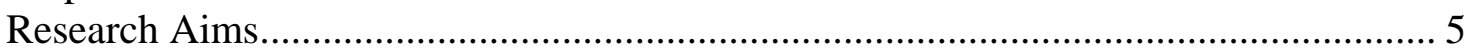

CHAPTER II: LITERATURE REVIEW ……………......................................... 7

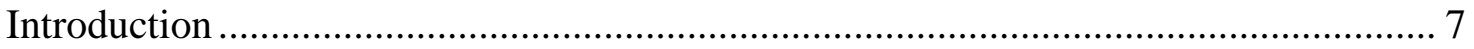

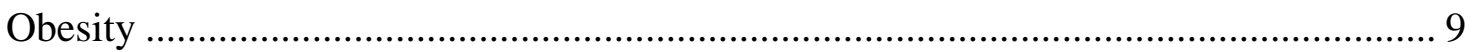

Measurilng Obesity ……………………...................................................... 10

Demographic Differences in Obesity Prevalence .................................................... 14

Obesity Risk Factors .......................................................................................... 16

Relationship Between Obesity and Alcohol Use ................................................. 22

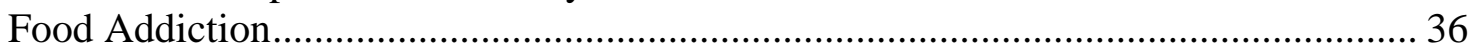

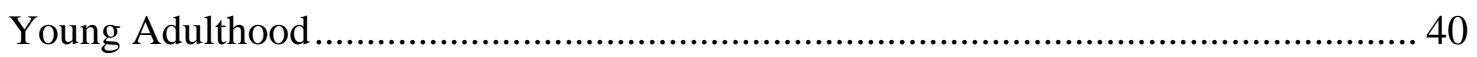

Theoretical Background ................................................................................... 40

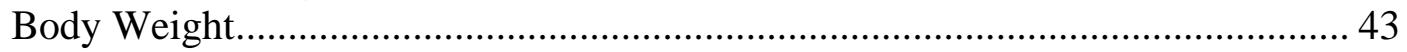

Alcohol Use ............................................................................................... 44

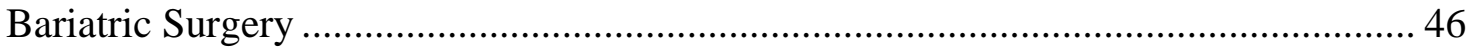

Governing Boards \& Policies .................................................................................. 48

Pre-surgical Psychological Evaluation................................................................ 52

Post-Surgical Issues..................................................................................... 53

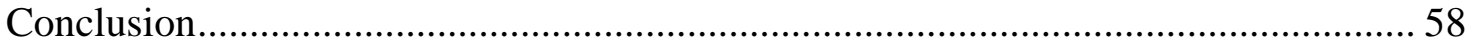

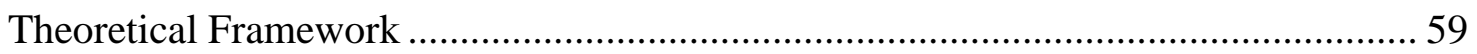

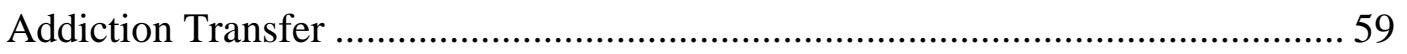

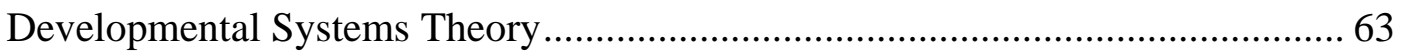

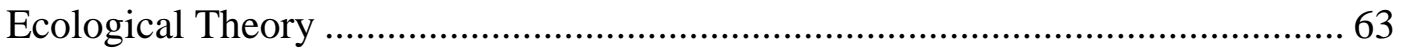

Integrated Developmental Systems and Ecological Theory...................................... 64

Systematic Review Of The Published Literature On Alcohol And Drug Use Among

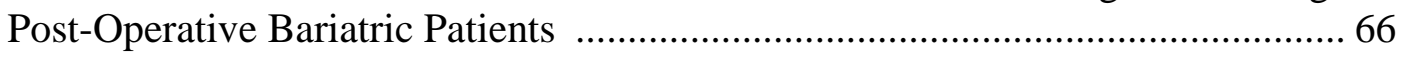

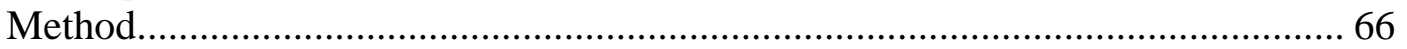

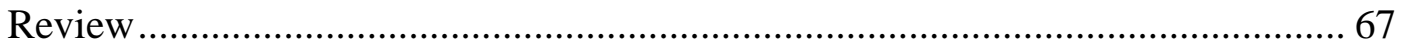

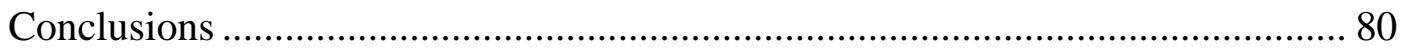

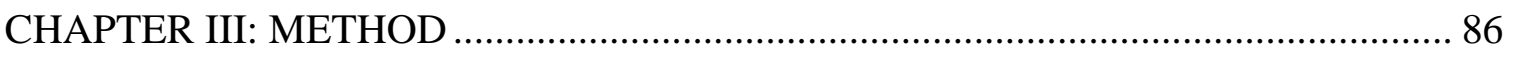

Overview of Mixed Methods Research Design .......................................................... 86

Sequential Explanatory Mixed Methods Design......................................................... 87

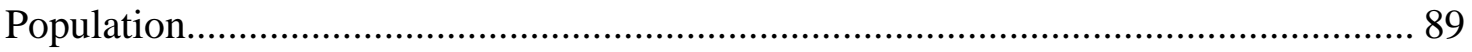

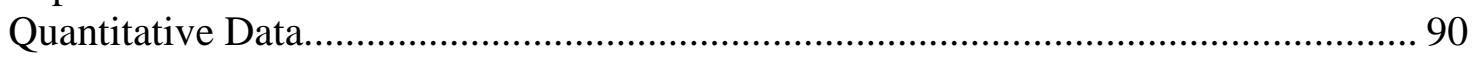

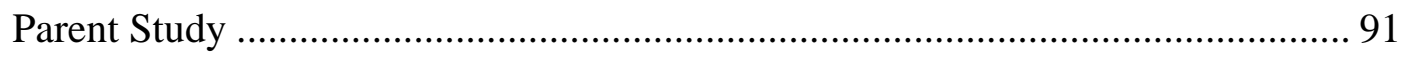

Contributions of the researcher to the parent study................................................. 91

Quantitative Data Collection Measures......................................................................... 93

Quantitative Data - Management \& Analysis ................................................................. 93 
Qualitative Data Collection .............................................................................. 96

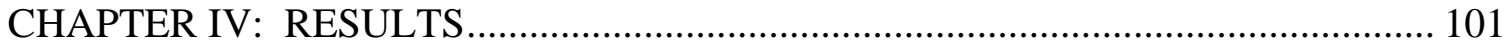

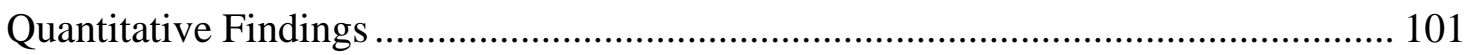

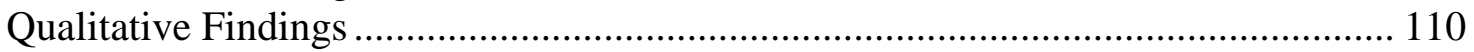

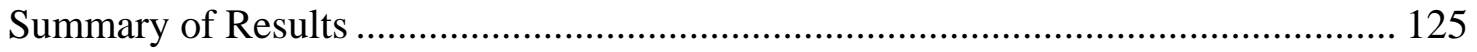

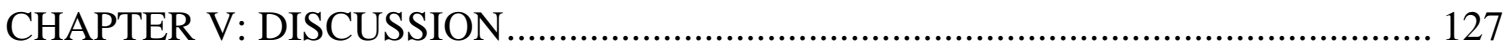

Quantitative Findings ...................................................................................... 128

Conclusion for Quantitative Results .................................................................. 131

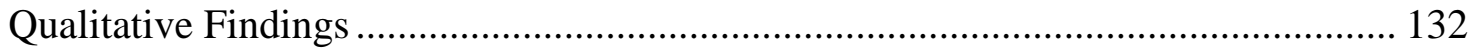

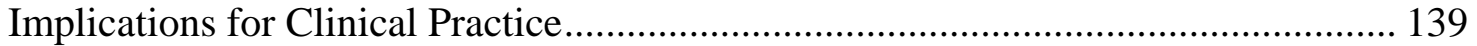

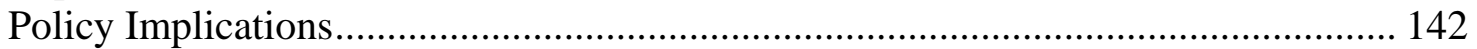

Implications for Future Research .................................................................... 143

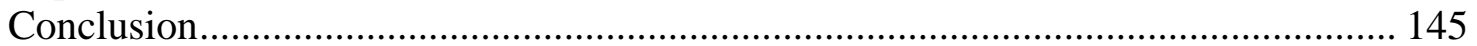

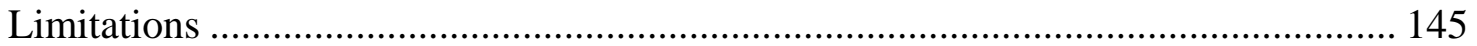

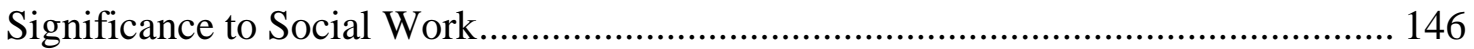

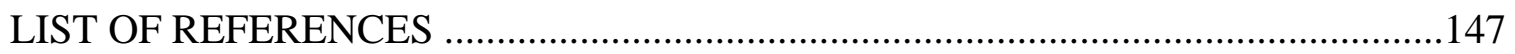

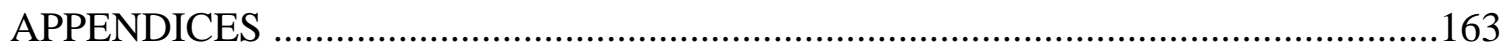

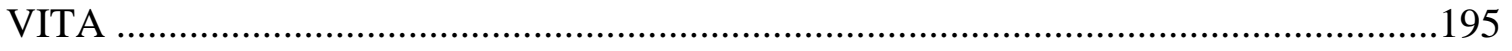




\section{LIST OF TABLES}

TABLE

PAGE

1. Summary of the Literature Investigating the Relationship of Alcohol on Body Weight

2. Characteristics of Published Studies Examining Alcohol and Illicit Drug Use in Post- Bariatric Populations. .164

3. Summary Of Results Among Studies Examining Alcohol And Illicit Drug Use In Post-Bariatric Populations

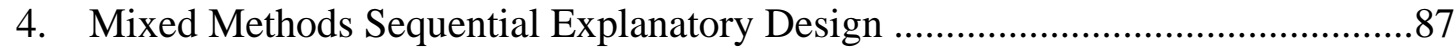

5. Demographic Characteristics of the Sample ...................................................101

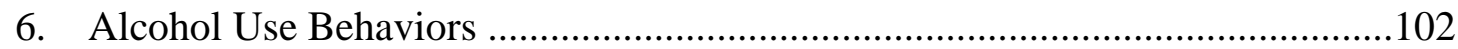

7. Regression Results for Number of Days of Alcohol Use in Past 30 Days .........104

8. Regression Results for Number of Days of Drinking to Intoxication in Past 30 Days .106

9. Regression Results for Number of Days of Binge Drinking in Past 30 Days ....107

10. Frequency Statistics for Change in Alcohol Use Following WLS ...................108

11. Themes Surrounding Changes in Alcohol Use Following WLS ......................110

12. Qualitative Interview Participant Demographics .........................................112 


\section{ABBREVIATIONS AND ACRONYMS}

AUD

AGB

SG

RYGB
Alcohol use disorder

Adjustable gastric band procedure

Sleeve gastrectomy procedure

Roux-en-y-gastric bypass procedure 


\section{CHAPTER 1: INTRODUCTION}

Weight loss surgery (WLS), or bariatric surgery, is considered the most effective, long-term treatment for morbid obesity (Belle et al., 2007). The number of weight loss surgeries performed in the United States has more than tripled over the past two decades (Flegal, Carroll, Kit, \& Ogden, 2012; Nguyen et al., 2011; Reedy, 2009), with approximately 220,000 surgeries currently being performed each year, compared to only 16,000 in 1990 (Davis, 2009). The health benefits of bariatric surgery such as the reversal of type 2 diabetes and other cardiometabolic disease risk factors have been welldocumented in both adults (Buchwald et al., 2004; de la Cruz-Munoz et al., 2011; Gomez-Meade, Lopez-Mitnik, Messiah, Carrilo, \& de la Cruz-Munoz, 2013) and adolescents (Messiah et al., 2013a; Treadwell J.R. \& Schoelles, 2008).

Recent research reveals problematic alcohol use among some bariatric surgery patients, and suggests the following risk factors are associated with post-operative problematic drinking: (a) the prevalence of a lifetime alcohol use disorder (AUD) prior to WLS, having WLS at a young age, and the Roux-en-Y gastric bypass (RYGB) procedure (where the digestive tract bypasses a large portion of the stomach) which results in an increased post-surgical sensitivity to alcohol (Conason et al., 2012; King et al., 2012a; Suzuki, Haimovici, \& Chang, 2012). In fact, recent research revealed that in women who had the RYGB procedure, two alcoholic beverages produced a Blood Alcohol Content of equivalent to four drinks; thus, the RYGB has been shown to double the effects of alcohol (Pepino et al., 2015). Post-operative recommendations set forth by the American Society for Metabolic and Bariatric Surgery advises all patients to avoid alcohol 
consumption for six months after surgery to facilitate post-surgical healing, weight loss and prevent macro-nutrient deficiencies (Mechanick et al., 2013). Recommendations also include that patients at risk for post-operative alcohol use disorders (AUD) avoid any alcohol use indefinitely after surgery. However, research indicates that as many as $80 \%$ of patients demonstrating problematic drinking behaviors still undergo bariatric surgery (Kudsi et al., 2013).

There is reason to believe both (a) young adult and (b) Hispanics/Latino bariatric surgery patients could be particularly prone to problematic drinking post-surgery, as both demographic groups represent an elevated risk of problematic alcohol use in the general population. Specifically, according to epidemiological studies, young adults are particularly vulnerable for problematic alcohol use (Brown et al., 2008; Centers for Disease Control and Prevention, U.S. Department of Health and Human Services, 2013; Chen \& Kandel, 1995; National Institute on Alcohol Abuse and Alcoholism, 2006). The literature also indicates that Hispanics/Latinos, compared to non-Hispanic whites, are at an increased risk of experiencing negative alcohol-related health consequences when they drink, including psychosocial, legal, and health problems (Greenfield, 2001; Mulia, Ye, Greenfield, \& Zemore, 2009; Yoon, Yi, Grant, \& Dufour, 2001). Thus, collectively, Hispanic/Latino and/or young adult bariatric surgery patients might present with compounded risk factors for alcohol use problems following bariatric surgery. However, to date, there has been no in-depth assessment of alcohol use among young adult bariatric surgery patients. Thus, the following research quantitatively and qualitatively assesses 
alcohol use in post-operative young adult, predominantly racial/ethnic minority bariatric surgery patients.

Moreover, non-Hispanic blacks, Hispanics, and American Indians are disproportionately affected by obesity and morbid obesity in the United States (Flegal et al., 2012; Schiller, Lucas, \& Peregoy, 2012). Thus, racial/ethnic minorities represent the most eligible population for bariatric surgery and will likely represent an increasing demographic that will undergo bariatric surgery, as has been reported in the literature (Pickett-Blakely, Huizinga, \& Clark, 2012).

Young adults may represent an emerging demographic undergoing bariatric surgery as improved health outcomes and weight loss have been documented in younger populations (Messiah et al., 2013a; Treadwell J.R. \& Schoelles, 2008)' and increased weight loss has been associated with having bariatric surgery at a younger age(Contreras, Santander, Court, \& Bravo, 2013). Young adults and racial/ethnic minorities such as Hispanics/Latinos and African Americans also might be at heightened risk for problematic drinking post-surgery. In the general population, adolescents and young adults are especially vulnerable to binge drinking and abusing alcohol (Brown et al., 2008; Chen, Dufour, \& Yi, 2005; SAMHSA, 2006). In fact, among women, non-Hispanic whites and Hispanics 18 to 34 years old demonstrate the highest prevalence of binge drinking (Centers for Disease Control and Prevention, 2013) compared to other demographic groups. Moreover, in the bariatric surgery literature, younger age appears to be associated with increased alcohol use among bariatric surgery patients (King et al., 2012). Furthermore, Hispanics/Latinos are also more likely to experience negative 
alcohol-related health and psychosocial consequences when they drink, compared to nonHispanic whites (Greenfield, 2001; Mulia et al., 2009; Yoon et al., 2001).

\section{Purpose of the Research}

This sequential explanatory mixed methods study sought to address the current lack of knowledge concerning alcohol use among young adult WLS patients. Simultaneously, this research also sought representation of racial/ethnic minorities, a demographic that has also been underrepresented in the bariatric surgery literature to date. By employing qualitative and quantitative analyses, the study provided an in-depth examination of the contexts and phenomenology surrounding alcohol use and problematic alcohol use among young adult, WLS patients. In turn, risk and protective factors were elucidated which can inform pre- and post-operative counseling and interventions for WLS patients with alcohol use problems.

\section{Research Aims}

Aim 1: Determine current (past 30 day) frequency of alcohol use as well as alcohol use disorder diagnoses among post-operative young adult bariatric surgery patients. Specifically assess frequency of alcohol use, binge drinking, and frequency of drinking to intoxication, as well as current and lifetime alcohol use disorder diagnoses (assessed using DSM-IV diagnostic schemes).

Aim 2: Assess psychosocial and physical predictors of past 30 day (post-surgical) frequency of alcohol use, including binge drinking, and drinking to intoxication. Specifically examine if the aforementioned drinking patterns are associated with the following independent variables, informed by the bariatric literature: (a) the RYGB 
surgical procedure, (b) prevalence of a lifetime (pre-surgical) AUDs, (c) time since surgery, and (d) age.

Aim 3: Analyze self-reported changes in alcohol use and subjective physiological responses to alcohol after bariatric surgery through quantitative and qualitative methodologies.

Aim 4: Explore factors surrounding post-operative alcohol use, through semi-structured, in-depth interviews of respondents who reported an increase in alcohol consumption following WLS and/or those who report regular alcohol consumption ( $\geq 3$ times per week) post WLS. 


\section{CHAPTER II: LITERATURE REVIEW}

\section{Introduction}

Obesity remains one of the leading health concerns in the United States, and is also associated with serious medical problems such as hypertension, type 2 diabetes, cardiovascular disease, and certain cancers (Henstrud, 2006). In 2013, the American Medical Association classified obesity as a disease. While some experts believe that this classification will help alleviate the discrimination and stigma associated with obesity as well as promote varying treatments for obesity (i.e. counseling) (Hurt et al., 2014), the official designation of obesity as a disease remains controversial (Hofmann, 2015; Hurt et al., 2014).

Obesity and severe obesity are defined in terms of Body Mass Index (BMI), a calculation of weight in kilograms divided by height in meters squared(Flegal et al., 2012). A BMI of $30 \mathrm{~kg} / \mathrm{m}^{2}$ or greater is considered obese, and a BMI of $40 \mathrm{~kg} / \mathrm{m}^{2}$ and above is considered severely obese (Flegal et al., 2010). Of note, “severe obesity” and "morbid obesity” are often used interchangeably in the literature. However, due to the judgmental tone of the term "morbid obesity," the term "severe obesity" will be used throughout this dissertation.

Bariatric surgery, also referred to as weight loss surgery, is known as the most effective, long-term treatment for severe obesity (Belle et al., 2007), as behavioral and pharmacological interventions result in long-lasting weight loss in only $5-10 \%$ of the population (Mechanick et al., 2013). According to a recent meta-analysis, bariatric 
surgery results in a BMI loss between 12 to 17 points, even at 5 years post-surgery (Chang et al., 2013).

Individuals are eligible for bariatric surgery if they have a Body Mass Index $(B M I) \geq 35$ with obesity related comorbidities, or a BMI $\geq 40$ with or without obesity related comorbidities (Mechanick et al., 2013). In the United States, over 15\% of Americans have a BMI greater than 35, which includes $17.2 \%$ of females and $10.1 \%$ of males aged 20-39 (Flegal, Caroll, Kit, \& Ogden, 2010). According to the most recent population estimates, this means that almost 48 million people are considered to be obese in the United States (United States Census Bureau, 2014).

In addition to weight loss, the health benefits of bariatric surgery (e.g., the reversal of type 2 diabetes and other cardiometabolic disease risk factors) have been well established in adults (Buchwald et al., 2004; Gomez-Meade et al., 2013; Heneghan et al., 2013) and adolescents (De La Cruz-Munoz et al., 2013). Despite these salubrious results, emerging research suggests that bariatric surgery patients may be at a heightened risk for developing post-surgical alcohol use problems such as alcohol abuse and dependence (Conason et al., 2012, King et al., Suzuki et al., 2012).

Psychological and physiological explanations have been posited to help explained this heightened vulnerability for post-operative problematic alcohol use. First, the theory of "addiction transfer" has been suggested, meaning that one trades a (presurgery) addiction to food for an addiction to alcohol post-surgery (McFadden, 2010; Volkow et al., 2013; Wang et al., 2004). Second, physiological explanations posit that certain bariatric surgical procedures result in a smaller stomach, and consequently 
heightened sensitivity to alcohol (Hagerdorn, Encarnacion, Brat, \& Morton, 2007;

Klockhoff, Näslund, \& Jones, 2002; Woodard, Downey, Hernandez-Boussard, \& Morton, 2011).

This chapter reviews the current literature regarding (a) obesity, (b) the relationship between alcohol use and obesity, (c) food addiction, (d) young adulthood, (e) bariatric surgery and (f) drug and alcohol use among post-operative bariatric surgery patients. In addition, specific emphasis has been placed on investigating the literature/studies that includes racial/ethnic minorities. Furthermore, the guidelines regulating bariatric surgery in the United States, and the inclusion of Medicare and Medicaid participants, are also discussed.

\section{Obesity}

The latest estimates reveal that approximately $36 \%$ of adults and $17 \%$ of children and adolescents living in the United States are classified as being obese, defined as having a body mass index (BMI) of at least 30 (Flegal et al., 2012). Data specifically concerning young adults, defined in this dissertation as individuals aged 18-35, is limited; however the most recent estimate reveals 33.2\% of men and 31.9\% of women from ages 20-39 are obese (Flegal et al. 2012).

Severe obesity, also referred to as morbid obesity, is defined as having a BMI of at least $40 \mathrm{~kg} / \mathrm{m}^{2}$ and effects $6.3 \%$ of Americans. Furthermore, among high-income countries, the U.S. has the highest mean BMI for both men and women (Farzadfar et al., 2011). While the rates of obesity have recently plateaued, severe obesity continues to 
increase, with approximately 15 million people in the U.S. having a BMI of $40 \mathrm{~kg} / \mathrm{m}^{2}$ or greater (Ogden, 2012). The co-morbidities associated with obesity and severe obesity are numerous and severe, and include cardiovascular diseases, type 2 diabetes, and certain cancers (Guh et al., 2009). Moreover, a higher BMI is associated with greater risks and treatment complications (Finkelstein et al., 2012). Young adult females who are in their childbearing years could also be negatively impacted by obesity in terms of fertility and pregnancy outcomes (Bandealy \& Stahl, 2012; Levitsky, Misra, \& Boepple, 2009). Specifically, obesity is associated with an increased risk of pre-eclampsia, increases in cesarean delivery, gestational diabetes, and respiratory disease and infections (Bandealy \& Stahl, 2012).

Obesity is also associated with a reduced life expectancy. Compared to individuals in the normal weight category, a BMI of 35 to $40 \mathrm{~kg} / \mathrm{m}^{2}$ is associated with a reduced life expectancy of 5 years, and a BMI over 40 (severe obesity) is associated with a reduced life expectancy of nine years (Finkelstein et al., 2012). Approximately 112,000 deaths per year are associated with obesity in the United States (CDC, 2011). Because of the link between obesity and serious health implications, overweight and obesity are now recognized as leading public health challenges facing the United States and other industrialized nations (Burkhauser \& Cawley, 2008).

\section{Measuring Obesity}

As described earlier, obesity is commonly measured in terms of Body Mass Index (BMI), which is calculation that involves an individual's height and weight. However, BMI is often considered an imperfect measure of obesity, as it lacks specificity and 
sensitivity to factors that are important to consider when assessing body weight and health outcomes (Burkhauser \& Cawley, 2008; Rothman, 2008). Overall, BMI does not allow for differentiation between fat and fat-free mass such as muscle and bone.

Additionally, BMI in adults also not account for age and gender, which are also important factors when assessing body weight and health. With age, a person's body fat increases but muscle mass decreases; this might not be reflected in someone's height or weight (Rothman, 2008). Therefore, it is not uncommon to have a seemingly overweight elderly person who is considered normal or even underweight according to the BMI formula. Along the same lines - the opposite can occur; muscular individuals can also be lean with high muscle mass, and have a BMI score which describes them as overweight or obese (Rothman, 2008). Thus, BMI may misclassify obesity status.

BMI can also under-classify obesity, specifically in females. Burkhauser and Cawley (2008) report that when comparing percent body fat (PBF) and BMI when assessing obesity, $23.7 \%$ of females were obese according to BMI, but $70.4 \%$ were classified as obese when assessing PBF. A significant, yet less pronounced difference is also found with men: $19.0 \%$ of men were classified as obese according to BMI, but 43.4\% were classified as obese according to percent body fat (Burkhauser \& Cawley, 2008).

BMI might also over-estimate the racial/ethnic differences in the prevalence of obesity, specifically when comparing African Americans and non-Hispanic Whites. Burkhauser and Cawley (2008) found that on average African American females have approximately $3.5 \mathrm{~kg}$ more of fat free mass (muscle, bone, fluid, etc.) compared to non- 
Hispanic white females, and African American males have approximately $1.3 \mathrm{~kg}$ more fat free mass compared to non-Hispanic white males. Because BMI does not distinguish between fat-free mass and body fat, there are significant differences when comparing BMI-based obesity rates between these two racial/ethnic groups (Burkhauser \& Cawley, 2008).

Furthermore, when using BMI to define obesity, there was an $11.9 \%$ difference between African American and non-Hispanic White females, compared to only a $5.1 \%$ difference in obesity when using percent body fat. 34.1\% of African American females and $22.1 \%$ of non-Hispanic white females were classified as obese using BMI, compared to $74.8 \%$ of African American females and $69.7 \%$ of non-Hispanic white females (Burkhauser \& Cawley, 2008). Even more compelling, the difference is greater in males, with non-Hispanic white men having a higher rate of obesity compared to African American men when using percent body fat. According to BMI measurements, $19.4 \%$ of African American males, and 18.9\% of non-Hispanic White males are obese. However, when using percent body fat, 29.1\% of African American males are classified as obese, compared to $45.4 \%$ of non-Hispanic white men. In other words, when using BMI, there was a $0.45 \%$ difference in obesity rates but when using percent body fat, that difference was $16.3 \%$, with non-Hispanic white males have a higher prevalence of obesity (Burkhauser \& Cawley, 2008).

Alternative obesity measures to BMI include percent body fat (body fat divided by total mass), total body fat, waist circumference, and waist-to-hip ratio (Burkhauser \& Cawley, 2008). Because fat secretes the hormones resistin and leptin, and may determine 
how much of these hormones are released, obesity is often directly associated with Type II diabetes and cardiovascular diseases (Trayhurn \& Beattie, 2001). Thus, it has been suggested that total body fat could be the most effective measure of obesity especially when examining health risks.

Research also reveals that location of fat is also important in assessing health risks. Specifically, abdominal fat, or stomach fat, which is the fat located around the internal organs, has been associated with an elevated risk of mortality (Bray, Bouchard, \& James, 1998). Abdominal fat can be assessed by measuring waist-to-hip ratio or waist circumference. While waist to hip ratio compared to BMI has been specifically found to be a better predictor of heart attacks, NIH recommends waist circumference versus waistto hip ratio to measure abdominal fat (Burkhauser \& Cawley, 2008).

Although total body fat seems to have the most support in the literature as an indicator of obesity (Burkhauser \& Cawley, 2008), there is no agreement on the ideal measure of overweight and obesity. Moreover, BMI remains the most commonly used measure of obesity in part because it relies solely on height and weight and can be easily measured and reported. Thus, it is important to consider the varying factors that can affect BMI, and that BMI does not differentiate between fat and lean muscle mass (Burkhauser \& Cawley, 2008; Rothman, 2008). Nonetheless, because BMI is the most easily reportable measurement of body weight and is the measure most often cited in the literature, BMI will be the primary index of obesity throughout the remainder of the paper. 


\section{Demographic Differences in Obesity Prevalence}

Race/Ethnicity. There are remarkable racial and ethnic disparities in the prevalence of obesity in the United States. Based on data from the National Health and Nutrition Examination Survey (NHANES), which utilized BMI as the measurement for obesity, Flegal and colleagues (2012) found non-Hispanic black men and women to have among the highest rates of obesity in the United States (58.5\% of women and $38.8 \%$ of men), followed by Mexican-Americans (44.9\% of women and 36.6\% of men), Hispanics (41.4\% of women and 37.0\% of men), and finally non-Hispanic whites (32.2\% of women and $36.2 \%$ of men). It is not clear why the authors analyzed the obesity rates of Mexican-Americans apart from other Hispanic-Americans. Flegal and colleagues did not report on the obesity rates of American Indians and Asian ethnic groups, however, data from the National Health Interview Survey found that American Indians have among the highest age-adjusted obesity rates (40.8\%), followed by 38.9\% of African Americans, 34.7\% of Hispanics, 27.2\% of whites, and 9.3\% of Asians (Schiller et al., 2012).

Race/Ethnicity and Age. Significant ethnic differences in the prevalence of obesity also exist among children and adolescents. Approximately $24.3 \%$ of nonHispanic black children and adolescents are classified as obese, followed by $21.2 \%$ of Hispanic children/adolescents, and 14\% of non-Hispanic white children and adolescents (Ogden et al., 2012). Data concerning racial/ethnic differences in obesity among young adults is limited, as most population based studies combine younger and older adults (Koebnick et al., 2012). However Flegal and colleagues (2012) provide obesity data for adults aged 20-39 years, and reveal that non-Hispanic Black women have the highest 
obesity rates (56.2\%), followed by Mexican-American females (37.8 \%), then nonHispanics Black men (35.8\%), non-Hispanic white men (34.5\%), Hispanic females (34.4\%), Mexican American males (32.7\%), Hispanic males (30.8\%), and finally nonHispanic white females (26.9\%) (Flegal et al., 2012).

Socioeconomic status. Recent research also reveals that children of low socioeconomic status have higher rates of obesity than youth of high socioeconomic status (Frederick, Snellman, \& Putnam, 2014). From 2009 -2010, 26\% of US adolescents between the ages of 12-17 whose parents had no more than a high school education were obese. This can be compared to an obesity rate of only $7 \%$ among adolescents whose parents had at least a four- year college degree. Lack of exercise was attributed to this health disparity, with low-income youth less likely to participate in sports and other physical activities (Frederick et al., 2014). Thus, income, education, as well as race and ethnicity could all play a role in obesity.

Socioeconomic status and race/ethnicity. In her book, What's Wrong with Fat? (2013), Abigal Saguy explores varying "blame frames” that surround obesity. She remarks that minority culture in the U.S., as well as socio-economic status, is often attributed to promoting obesity. For example, according to obesity researcher Xavier PiSunyer:

Now a lot of the obesity problem in America or more of it is in the minority groups and more in the poorer groups, and it may be that they have other priorities. You know, some woman who's living in the housing projects and has no husband and is trying to take care of four kids and is now off welfare and has 
to work and has all kinds of problems. For her, diet is not [a priority]. In fact, food is the one pleasurable thing she can afford, so it's not high priority in their lives. I'm not saying they're wrong, but I'm saying I don't think they're really connected to the idea that, you know, they need to lose twenty-five pounds, and so they don't try it (Saguy 2013, p.75).

As will be explored in the following section, varying environmental, cultural, and biological factors can all play a role in promoting obesity.

\section{Obesity Risk Factors}

Environment. Risk factors for obesity can have environmental, biological, and socio-cultural foundations. Environmental risk factors can be seen in the United States and other industrialized nations where affordable, easily accessible, and palatable foods are prevalent and sedentary office jobs and private transportation are common, resulting in minimal physical activity (Van Buren \& Sinton, 2009). Furthermore, it is common for individuals living in the United States to often eat at their computer, while driving, or while conducting other activities that promote over-eating and the consumption of prepackaged foods "on the go" (Saguy, 2013). In addition, foods high in fat and carbohydrates are often the cheapest, most easily available, and most desired (Saguy, 2013; Swencionis \& Rendell, 2012). Thus, factors in a socio-cultural environment such as the abundance of readily available, low-cost, high calorie food, and the high use of personal automobiles and long-work hours all contribute to an environment that promotes obesity—what is known as an obesogenic environment. 
Cultural factors. The literature indicates that culture can also impact obesity due to cultural influences on weight perception, norms attributed to ideal body types, and perceived health risks of obesity (Dorsey, Eberhardt, \& Ogden, 2009). Weight perception, which is the concurrence between self-perception of body weight and measured body weight, is associated with attempts at weight loss. Commonly, those viewing themselves as overweight are more likely to attempt to lose weight than those without this perception (Lemon, Rosal, Zapka, Borg, \& Andersen, 2009). Cultural differences in weight perception have been well documented in the literature (Dorsey, Eberhardt, \& Ogden, 2010; Hendley et al., 2011).

Specifically, in a study examining weight perception in African American and non-Hispanic white respondents, African-Americans were three times more likely to underestimate their weight compared to non-Hispanic whites (OR 3.1, 95\% CI 1.9-4.8) (Hendley et al., 2011). The difference was even larger for black women than for black men $(\mathrm{p}<.001) ; 62.3 \%$ of African-American women underestimated their weight, compared to only $21.5 \%$ of non-Hispanic white women, while $68.1 \%$ of black men underestimated their weight, compared to $53.5 \%$ of non-Hispanic white men ( $p=.08)$.

There are also cultural differences in accurate weight estimation among adolescents. Park (2011) investigated weight overestimation and underestimation among 87,418 high school students residing in Minnesota. Data reveal that African-American and Hispanic females were more likely to underestimate their weight, while Asian Americans were more likely to overestimate their weight (odds ratios=.379, .889, 1.259, respectively; $\mathrm{p}<.05)(P a r k, 2011)$. Krauss and colleagues (2012) reported similar findings. 
Among a sample of female adolescents $(n=5,035)$ who responded to the National Survey of Youth 1997, African-American and Hispanic adolescents were significantly more likely to underestimate their weight compared to non-Hispanic white respondents (28.6\%, $15.9 \%$, and $12.9 \%$ respectively; $\mathrm{p} \leq .05$ ) (Krauss, Powell, \& Wada, 2012).

Another study examined young adult (18-25) females and indicated a higher prevalence of weight misperception among African-American and Hispanic women than among non-Hispanic white women (Rahman \& Berenson, 2010). Specifically, significantly more overweight African-American (28.2\%) and Hispanic (24.7\%) women misperceived their weight as "normal” weight compared to overweight non-Hispanic whites women (14.8\%) $(p<.001)$. Furthermore, normal-weight white women were more likely to consider themselves overweight compared to African-American women (16\% vs. 7\%,; OR 0.40; 95\% CI .22-.74) (Rahman \& Berenson, 2010).

Sivalingam and colleagues (2011) investigated self-recognition of obesity and the health risks attributed to obesity. Similar to previous findings, non-Hispanic Whites were more likely to self-report obesity than other racial/ethnic groups (Sivalingam 2011). Equally important, only 77\% of African Americans viewed obesity as a health problem compared to $90 \%$ of non-Hispanic whites and $88 \%$ of Hispanics $(\mathrm{p}<.001)$.

Cultural norms surrounding the ideal body type might also influence higher body weights in certain racial/ethnic groups, specifically among African-American females. The literature has found that despite higher body weights, African-American women are more comfortable with their weight than non-Hispanic white women (Powell \& Kahn, 1995). In fact, Powell and Kahn (1995) reported that non-Hispanic white women 
identified thinner body type as the ideal body size, and expressed more concern about their weight and dieting than African American women. In addition, research also suggests that overall, African-American women are less likely to view excess body weight as negative compared to white women (Flynn \& Fitzgibbon, 1998; Powell \& Kahn, 1995). Reasons for varying beliefs surrounding body weight could also be driven by the desire to be sexually attractive. In fact, research suggests that African-American males are more likely to prefer females of a heavier body weight compared to nonHispanic white males (Flynn \& Fitzgibbon, 1998).

This appears to be corroborated in research investigating weight perception among young adult women. Chithambo and Huey (2013) analyzed data of 1,694 mainly young adult ( $\mathrm{M}=28.9$ years; $\mathrm{SD}=1.7$ years) overweight or obese African-American and non-Hispanic white women. The African-American sample was on average heavier, yet reported lower perceived body weight and viewed their bodies favorably, than compared to the non-Hispanic white sample, which demonstrated to have negative physical selfperception. In addition, there was an inverse relationship between BMI and self- rated attractiveness in the non-Hispanic white sample, but there was no relation between BMI and self-rated attractiveness in the African-American sample (Chithambo \& Huey, 2013).

While not all of the literature is conclusive on these issues (Grabe \& Hyde, 2006), the majority of the literature reveals that the "ideal body image" is defined differently based on racial/ethnic group (Flynn \& Fitzgibbon, 1998; Powell \& Kahn, 1995; (Stice, 1994). Overall, the majority of the literature reviewed here indicates that African Americans, specifically African American females, might experience less social 
pressure to be thin, and less prone to experiencing a negative body image, which can potentially contribute to acceptance and normalization of overweight and obesity (Flynn \& Fitzgibbon, 1998; Powell \& Kahn, 1995).

In conclusion, cultural differences surrounding weight perception and ideal body type can impact overweight and obesity. The majority of the literature reveals that, in the United States, Hispanics and non-Hispanic Blacks are more likely to consider their weight as normal weight, when in actuality they are classified as overweight according to BMI charts (Flynn \& Fitzgibbon, 1998; Powell \& Kahn, 1995). Furthermore, research reveals that African Americans consider the ideal body type to be heavier than the ideal body type as defined by non-Hispanic whites. These factors can all contribute to racial/ethnic differences in obesity rates (Flynn \& Fitzgibbon, 1998; Powell \& Kahn, 1995; Stice, 1994).

Biological Factors. Research reveals that there are genetic and hereditary components to obesity, with at least 32 genes associated with BMI (Speliotes et al., 2010). However, due to environmental confounding factors, the relative genetic contribution to obesity remains difficult to ascertain (Hill \& Melanson, 1999). The literature investigating the variance of genetic factors is mixed, with studies on identical twins indicating genetic factors account for anywhere between $50-70 \%$ of the variance in BMI, and family studies indicating genetic factors account for $25-50 \%$ variance in body weight (Hill \& Melanson, 1999).

Stress is also associated with overweight and obesity. Specifically, stress affects hormones such as cortisol, epinephrine, and norepinephrine, which are associated with 
increased abdominal fat (Wardle, Steptoe, \& Lipsey, 2000). Studies also indicate that stress increases food consumption, and those experiencing stress crave more high-fat, high sugar foods (Wardle et al., 2000). Thus, an abundance of unhealthy and affordable food coupled with high stress levels and little time for physical activity, all can contribute to obesity and an obesogenic environment (Wardle et al., 2000).

Ghrelin. Ghrelin is a gut hormone that was discovered in 1999, and is nicknamed the "hunger hormone” (Schellkens, Dinan, \& Cryan, 2013). Overall, the ghrelinergic system affects both the peripheral and central nervous system and regulates appetite and food intake. Emerging research is also suggesting that ghrelin is involved in stressinduced food consumption and impacts the reward pathways. Ghrelin gene polymorphisms and mutations have also been associated with an increased obesity risk (Davenport \& Wright, 2013; Schellkens et al., 2013).

Ghrelin is also involved in satiety and sensations of hunger and meal initiations. In fact, humans are found to have high ghrelin levels just before they begin food consumption, and ghrelin levels decreases after food consumption. Studies examining ghrelin and its effect on obesity in animal models are inconclusive, but suggest that mice who have lower ghrelin levels are resistant to obesity onset from a high fat diet (Davenport \& Wright, 2013).

Interestingly, among bariatric surgery patients, greater weight loss has been documented in patients who undergo malabsorptive surgeries, which surgically reduce the size of the stomach, versus surgeries that just restrict food entering the stomach (i.e. lap band procedure)(Buchwald et al., 2004; Buchwald, 2010). Some post-surgical 
patients report sensations of feeling full, which they often describe as a unique feeling and one they have not experienced in a long time. It is hypothesized that gut hormones, such as ghrelin, are reduced with the surgery on the stomach, which results the patient reporting increased satiety with lower amounts of food compared to pre-surgery (Davenport \& Wright, 2013; Schellkens et al., 2013).

Considering the discovery of ghrelin and its effects on appetite, reward, and feelings of satiety, it is no surprise that pharmaceutical companies are in the process of developing medications that target ghrelin for the treatment of obesity (Davenport \& Wright, 2013). The literature indicates that ghrelin could also play a role in alcohol consumption, which is discussed later in this chapter.

Overall, the literature indicates that biological factors such as genetics and hormones can contribute to obesity. However, while it is not known how much these factors account for obesity, it is clear that biological and genetic factors are associated with, but not solely responsible for, obesity (Davenport \& Wright, 2013; Egger \& Swinburn, 1997; Hill \& Melanson, 1999).

\section{Relationship Between Obesity and Alcohol Use}

The literature investigating the association between alcohol use and body weight remains mixed, with the majority of epidemiological studies suggesting an inverse relationship between BMI and alcohol use. In other words, alcohol consumption is associated with an overall lower body weight (Breslow \& Smothers, 2005; Duncan, Grant, Bucholtz, Madden, \& Heath, 2009; Kleiner et al., 2004). Conversely, however, the 
majority of studies utilizing short-term laboratory data suggest that alcohol is ingested as additional calories, leading to higher caloric intake in the short-term (Breslow, Chen, Graubard, Jacobovits, \& Kant, 2013; Yeomans, 2010). Since alcohol contains a significant source of calories (approximately 7.1 kilocalories per gram), it appears that alcohol users would be prone to excess caloric intake and weight gain (R. R. Smith et al., 2008). Exactly why longer-term studies do not indicate a positive relationship between alcohol use and weight gain remains unclear. The following sections review the literature that investigates (a) the association between alcohol and eating behaviors, and (b) the association between alcohol use and body weight.

Alcohol and Eating Behaviors. Studies investigating the effects of alcohol on appetite commonly use a “pre-load” design, which requires research participants to drink either an alcoholic beverage or a control (non-alcoholic) drink before a meal. Participants then have free control over how much they consume during the meal (Breslow et al., 2013). A review article investigating the existing literature on alcohol and food consumption reveals that alcohol often adds additional calories to daily overall caloric intake, meaning participants do not eat less because they consumed alcohol (Yeomans, 2010).

Breslow and colleagues (2013) found that diets of drinkers were poorer on drinking days than non-drinking days, with both men and women consuming more calories and fats on drinking days. Specifically, among a sample of 1,864 total participants, for men, calories from alcohol constituted 264 of the 433(61\%) excess kcal, with the rest of calories derived from food and/or nonalcoholic components of alcoholic 
beverages (i.e. mixers). For women, the alcohol in alcoholic beverages constituted 206 kcal (69\%) of the excess calories, with no higher calorie intake from food. The sample was classified as moderate drinkers, on average consuming 3- 7 drinks a week (Breslow et al., 2013). These findings support prior research, which revealed a positive relationship between alcohol consumption and calorie consumption in both men and women (Yeomans, 2010).

Researchers have investigated the effect of alcohol on restrained eaters, or individuals who are making a cognitive effort to limit food intake in order to control body weight. It has been posited that alcohol can lower inhibitions when it comes to restrained eating and have a disinhibitory effect on food consumption (Polivy \& Herman, 1976; Polivy \& Herman, 1976; Yeomans, 2010). Polivy and Herman (1976) found that the belief that alcohol was consumed and not actual alcohol consumption (i.e., "alcohol expectancies”) was associated with increased food consumption in restrained eaters (Polivy \& Herman, 1976). Another study found that among men, alcohol increased appetite among unrestrained eaters, but not among restrained eaters (Yeomans, 2010). Other research has found that alcohol has no effect on women who describe themselves as restrained eaters (Ouwens, Van Strein, \& van der Staak, 2003; Yeomans, 2010). Thus, the data examining the effects of alcohol on food consumption inhibitions suggest that alcohol might increase food consumption in some unrestrained and restrained eaters.

Alcohol and Hunger. Alcohol can also enhance food pleasantness, and potentially have an "appetizer effect” by increasing hunger early into a meal (Yeomans, Hails, \& Nesic, 1999). One physiological explanation is that alcohol triggers gastro- 
intestinal signaling systems that are normally activated by food. Specifically, ghrelin, the hunger hormone described earlier, has been shown to increase appetite and food consumption (Cummings, Frayo, Marmonier, Aubert, \& Chapelot, 2004; Wren et al., 2001). Thus, one theory is that alcohol might stimulate the release of ghrelin, resulting in increased appetite and food consumption (Yeomans, 2010).

Conversely, however, other studies investigating the effect of alcohol on ghrelin indicate that alcohol reduces ghrelin levels. Calissendorff and colleagues (2005) found that alcohol, compared to water, significantly reduced ghrelin levels in healthy human research participants (Calissendorff, Danielsson, Brismar, \& Röjdmark, 2005). Other researchers also documented decreased ghrelin levels in patients diagnosed with alcohol dependence who consumed alcohol within 24 hours (Badaoui et al., 2008). While alcohol might reduce ghrelin during acute alcohol intake, ghrelin has been shown to increase during periods of abstinence from alcohol consumption among individuals diagnosed with alcohol dependence (Badaoui et al., 2008; Leggio, Schwandt, Oot, Dias, \& Ramchandani, 2013). Thus, research suggests that alcohol increases hunger and makes food more appetizing. However, the role of ghrelin in alcohol-induced hunger remains to be elucidated.

Alcohol and body weight. The relationship between alcohol and body weight is complex. As discussed earlier, short-term data correlates alcohol use with increased caloric intake, but longer-term data indicates that alcohol use might be a protective factor against obesity (Duncan et al., 2009; Kleiner et al., 2004; Wang et al., 2003). Furthermore, research suggests that gender (Lahti-Koski, Pietinen, Heliovaara, \& 
Vartiainen, 2002), type of alcoholic beverage consumed (Bendsen et al., 2012; Thomson et al., 2012), and drinking patterns (Tolstrup et al., 2005) affect the relationship between alcohol and body weight.

Kleiner and colleagues (2004) investigated the relationship between BMI and alcohol use by analyzing charts of 298 female patients who were a part of a weight management program. An inverse relationship between BMI and alcohol use was revealed. The more obese a patient was, the less alcohol they were likely to consume. Patients with a BMI of $50 \mathrm{~kg} / \mathrm{m}^{2}$ or greater (considered "super obese”) were significantly less likely to consume alcohol in the past year. Specifically, nearly $63 \%$ of patients who were in the overweight but not obese category (BMI $\leq 29 \mathrm{~kg} / \mathrm{m}^{2} ; \mathrm{n}=8$ ) used alcohol in the past year, compared to $47.6 \%$ of patients considered to be obese (BMI of $30-39 \mathrm{~kg} / \mathrm{m}^{2}$; $\mathrm{n}=84$ ); $41.8 \%$ of patients considered severely obese (BMI $40-49 \mathrm{~kg} / \mathrm{m}^{2} ; \mathrm{n}=110$ ), and finally only $35.4 \%(n=96)$ of those considered super obese reported using alcohol in the past year (Kleiner et al., 2004). Age and socioeconomic status were positively associated with frequent drinking ( $\mathrm{p}<.05$ for both).

Gearhardt and Corbin (2009) also observed an inverse relationship between alcohol use and BMI among both men and women. The authors examined data from 37,259 participants from the National Epidemiologic Survey on Alcohol and Related Conditions (NESARC). Participants with obesity reported using alcohol significantly less frequently and in lower quantities than normal and overweight participants. Patients with severe obesity also reported alcohol use less frequently and in lower quantities than normal weight, overweight, and obese patients (Gearhardt \& Corbin, 2009). 
One of the most recent investigations of the relationship between BMI and alcohol use also suggests an inverse relationship. Barry and colleagues (2015) investigated alcohol use and BMI among U.S. college students $(\mathrm{N}=27,774)$ and found that not only did alcohol users have significantly lower BMIs than non-alcohol users $\mathrm{t}(26,968)=5.55 \mathrm{p}<.001$, but frequency of drinking was also associated with BMI. Specifically, individuals who reported binge drinking in the past 30 days had significantly lower BMIs than those who did not report current binge drinking $\mathrm{t}(27,094)=2.64, \mathrm{p}<.001$ (Barry, Piazza-Gardner, \& Holton, 2015).

Pre-clinical research also corroborates the findings from human subjects' research, and suggests an inverse relationship between alcohol use and obesity. Smith and colleagues (2008) investigated the association between ethanol consumption and weight gain in female mice. Mice were given either water or a mixture of water and $20 \%$ alcohol. The mice were then scheduled to be on 1 of 3 diets for 20 weeks: (1) a 30\% calorie-restricted diet, (2) a low-fat diet, or (3) a high-fat diet. The mice on the high fat diet had the highest body weight. Most notably, even though the ethanol-administered mice consumed more calories, they did not have a higher body weight than the nonethanol consuming mice (Smith et al., 2008). Thus, taken together, the majority of the literature suggests that BMI and alcohol use have an inverse relationship.

Gender. It is important to recognize that gender differences might exist when examining the relationship between body weight and alcohol use, with men who drink alcohol perhaps more prone to weight gain than women who drink alcohol. Specifically, a study conducted in Finland with a sample of 24,604 participants found that men who 
drank alcohol at moderate or high levels were more at risk for obesity, whereas for women, non-drinkers were more at risk for obesity (Lahti-Koski et al., 2002).

Dallongeville and colleagues (1998) also revealed slight gender differences. The authors examined the relationship between amount of alcohol consumption and BMI among alcohol users in France. The sample consisted of 1778 men and 1730 women, predominantly all wine drinkers. Results revealed that among women, alcohol consumption was inversely correlated with BMI $(\mathrm{P}<.0001)$ and body weight $(\mathrm{P}<.00002)$. However, no association was found between alcohol intake and BMI and body weight among men (Dallongeville et al., 1998).

Types of alcoholic beverages. It has also been theorized that different types of alcoholic beverages (e.g., hard liquor, wine, beer) might contribute to varying results when examining the relationship between BMI and body weight. Thomson and colleagues (2012) investigated alcohol consumption and change in body weight in older adults, who were a mean age of 63 . Results reveal that those who moderately consumed alcohol had a reduced risk of becoming overweight or obese over the 7 year follow up period, compared to abstainers (hazard ratio, 0.65; 95\% CI, 0.58-0.73). In analyzing types of alcohol and their protective factors against overweight/obesity, wine (HR, 0.75 ; 95\% CI, 0.68-0.84) had the greatest protective association, followed by hard liquor (HR, 0.85; 95\% CI, 0.78-0.93), and finally beer (HR, 0.90; 95\% CI, 0.82-1.00) (Thomson et al., 2012).

Beer consumption has been associated with the deposition of abdominal fat, otherwise known as "beer belly” (Bendsen et al., 2012). A systematic review and meta- 
analysis investigated the literature to assess beer consumption and its relation to abdominal fat and overall obesity. In total, 35 observational and 12 experimental studies were included in the review (Bendsen et al., 2012). The majority of the observational studies observed either a positive association with beer consumption and abdominal fat or no association; some studies indicated that heavy beer consumption (over $500 \mathrm{ML} / \mathrm{day}$ ) might be positively associated with abdominal fat. The relationship between beer consumption and obesity, on the other hand, is less clear. The data indicate an inverse relationship or no association between beer consumption and general obesity. Overall, the authors concluded that while heavy beer consumption might be related to abdominal fat, there is inadequate scientific evidence to assess if beer intake at moderate levels is related to obesity (Bendsen et al., 2012). Taken together, the research suggests that wine might be associated with a lower risk for obesity than compared to other alcoholic beverages, specifically beer.

Drinking patterns. Drinking patterns are another factor to consider when examining the relationship between alcohol and obesity. The literature suggests that drinking in high frequencies might be associated with a lower risk for obesity. Tolstrup and colleagues (2005) found that the most frequent drinkers had the lowest odds of being obese. For men, the odds ratios (OR) for a high BMI among daily drinkers was .73 (95\% CI: 0.65-0.82), among men drinking on 5-6 days of the week, .87 (95\% CI: 0.77-0.98), 24 days of the week, 1.00 (reference, 1 day/week, 1.17 (95\% CI: 1.02-1.34), 1-3 days/month, 1.39 (95\% CI: 1.17-1.64), and less than 1 time per month 1.27 (95\% CI: 0.86-1.90). For women, the results were similar with an OR of .71 for daily drinkers 
(95\% CI 0.6-0.83) and 1.74 for women drinking less than 1 day per month (95\% CI 1.32-

2.30) (Tolstrup et al., 2005).

Breslow and colleagues (2005) reported similar results and indicated that drinking in high frequencies but lower quantities was associated with a lower risk for obesity. Among a sample of 37,000 drinkers, men and women who consumed alcohol the most frequently and in small quantities had the lowest BMIs. The authors posit that frequent drinkers might compensate for calories consumed from alcohol by eating less overall. Furthermore, non-frequent drinkers could be labeled as “social occasion” drinkers, and would perhaps be more likely to overeat when they drank. Clinical studies reveal that even infrequent overeating could lead to weight gain over time (Breslow \& Smothers, 2005).

Arif and Rohrer (2005) also suggest that frequency and quantity of alcohol can be associated with overweight and obesity. Among a sample of 8,236 respondents, current drinkers had a .73 times lower odds of obesity than non-drinkers (95\% CI: $0.55,0.97$ ). However, binge drinkers had a significantly greater odds of being overweight ( $\mathrm{OR}=1.45$; 95\% CI: 1.02-2.05) or obese (OR=1.77; 95\% CI: 1.18-2.65). This lends support to the existing literature that has established the correlation between binge eating and binge drinking, with binge eating being correlated to overweight and obesity (Lahti-Koski et al., 2002). Interestingly, consuming 2 drinks a day was associated with reduced odds of being overweight (OR=.83; 95\% CI: 0.61-1.14) and obesity (OR=.59; 95\% CI: 0.410.86). However consuming 4 drinks per day (the definition of binge drinking for women) was associated with a 30\% greater odds of being overweight (95\% CI: 1.00-1.68) and 
$46 \%$ greater odds of being obese (95\% CI: 0.98-2.14) (Arif \& Roher, 2005). Collectively, these studies suggest that frequent drinking, but not heavy or binge drinking is associated with a lower risk of obesity.

Family history of alcoholism. Interestingly, while the literature suggests that alcohol use demonstrates to have an inverse relationship with BMI, a family history of alcoholism, especially among women, could be a risk factor for obesity. Grucza and colleagues (2010) analyzed two large scale epidemiological surveys and report that women who had a biological parent and/or sibling with a history of alcohol use problems or alcoholism were nearly $50 \%$ more likely to be obese (OR=1.48, 95\% CI:1.36, 1.61; p $<.0001)$. This relationship remained significant even when adjusting for covariates such as smoking, alcohol use, and depression. The relationship between a family history of alcoholism and obesity was also significant in men, but had a weaker association (OR=1.26; 95\% CI: 1.14-1.38). However, there was no significant association between a family history of alcoholism and obesity in men after adjusting for covariates (Grucza et al., 2010).

The authors posit that obesity might be more prevalent among individuals at a heightened risk for addiction (i.e. those with a family history of alcoholism), in part due to the addictive qualities of food that might be present. Furthermore, earlier studies have also suggested an association between a preference for sweet foods and a familial history of alcoholism (Pepino \& Mennella, 2007). Thus, familial histories of alcoholism need to be taken into consideration when examining risk factors for obesity, as well as the relationship between obesity and alcohol use. 
Presence of Alcoholism. Conversely, however, it is important to note that the association between alcohol dependence and/or chronic alcohol abuse and lower body weight has been well documented. Reasons for this include that heavy and long-term alcohol use is believed to result in increased fat oxidation, or fat loss (Addolorato et al., 1998). Thus, the literature consistently indicates that alcohol dependence does not represent a risk factor for obesity; in fact, an individual with a diagnosis of alcohol dependence might be at risk for malnutrition and low body weight (Addolorato et al., 1998). Therefore, while a family history of alcoholism might be a risk factor for obesity, the literature indicates that an individual with alcohol dependence is more likely to be thin and malnourished than obese.

Alcohol use and body weight concerns. Several studies investigated the correlation between alcohol use and its association between an individual's concerns with their body weight, independent of actual body weight. For example, someone who views themselves as overweight or obese might turn to heavy alcohol use to help cope with any societal stigmatization they might face (Antin \& Paschall, 2011).

Adolescent and young adult females are particularly susceptible to being concerned with their body weight. In fact, the literature suggests that approximately $40 \%$ of 14-year-old girls and $83 \%$ of college age women report either trying or wanting to lose weight (Antin \& Paschall, 2011). Overall, the existing research indicates a positive relationship between body weight concerns and alcohol use. Page and colleagues (1995) found that high school students in the United States who reported being unhappy with their weight were more likely to use alcohol and/or get drunk than those who reported 
being satisfied or neutral with their weight(Page, Scanlan, \& Allen, 1995). However, directions of causality are not elucidated. Palmqvist and Santavirta (2006) revealed similar results among teenagers in Finland, with drinkers reporting a higher degree of weight dissatisfaction than non-drinkers (Palmqvist \& Santavirta, 2006). Finally, Chinese adolescents who viewed themselves as overweight were significantly more likely to consume alcohol than those perceiving themselves to not be overweight (Xie et al., 2006).

Antin and Paschall (2011) specifically examined weight perception, weight change intentions, and alcohol use among young adults. While, among women, there was no relationship between perceived weight and heavy drinking, weight change intentions (trying to lose weight) was positively related to binge drinking. Conversely, this relationship was the opposite for men. Men who perceived themselves as overweight were significantly less likely to binge drink, with no relationship between weight change intentions and drinking among men. There was no relationship between BMI and alcohol use in both men and women (Antin \& Paschall, 2011). Thus, these findings corroborate earlier research that indicates women who are dissatisfied with their weight might be more likely to consume alcohol or binge drink than those who are satisfied with their weight. Conversely, for men, Antin and Paschall (2011) refute previous findings (Page et al., 1995; Palmqvist \& Santavirta, 2006; Xie et al., 2006) as an inverse relationship between perception as overweight/obese and binge drinking was found.

Summary of the association between alcohol use and obesity. The literature indicates that alcohol use among the general population is associated with both increased 
caloric intake and increased hunger in the short-term. However, in clinical and preclinical populations, there appears to be an inverse relationship between alcohol use and body weight (Kleiner et al., 2004; Gearhardt \& Corbin, 2009). As delineated in Table 1, gender, type of alcoholic beverage consumed, and drinking patterns also affect the relationship between alcohol and obesity. Moreover, individuals with a family history of alcoholism are more at risk for obesity than those without a family history of alcoholism. Women who are unhappy with their weight or who are trying to lose their weight might be more at risk for alcohol use and/or problematic drinking 
Table 1

Summary of the Literature Investigating the Relationship of Alcohol on Body Weight

Variable

Relationship

Short-term caloric intake

Hunger

Overall relationship between
Alcohol can result in increased caloric intake

Alcohol can increase hunger

Yeomans et al., 1999;

Cummings et al. 2004

Duncan et al., 2009; Kleiner et al., 2004; Smith et al., 2008

between BMI/body alcohol use and BMI/weight

Type of alcohol consumed and risk of overweight/obesity weight and alcohol use, especially among women

Wine demonstrates the greatest protective factor against obesity; beer might be associated with greater abdominal fat but not overall obesity

Drinking patterns
Drinking regularly but in low quantities ( $\geq 2$ per day) might be associated with lower risk of obesity. However, binge drinking might be associated with an increased risk for being overweight.
Thomson et al., 2012; Bendsen et al., 2012

Breslow et al., 2005; Arif \& Roher, 2005 


$\begin{array}{lll}\begin{array}{l}\text { Family history of } \\ \text { alcoholism }\end{array} & \begin{array}{l}\text { Increased risk for } \\ \text { obesity }\end{array} & \text { Gruzca et al., } 2010 \\ \begin{array}{l}\text { Presence of alcoholism } \\ \text { (alcohol dependence) }\end{array} & \text { Low risk of obesity } & \text { Addolorato et al., } 1998 \\ & & \\ \text { Body weight concerns } & \begin{array}{l}\text { Women who express } \\ \text { "weight change }\end{array} & \text { Antin and Paschall, } 2011 \\ & \begin{array}{l}\text { intentions” might be } \\ \text { more vulnerable to } \\ \end{array} & \\ & & \\ & & \end{array}$

\section{Food Addiction}

Biomedical Research. Neurobiological data offers some support for humans’ ability to become addicted to food, just as they are able to become addicted to a drug. (Volkow, Wang, Fowler, \& Telang, 2008). First, rewarding foods (i.e. foods high in fat and/or sugar), have the same reinforcing characteristics similar to drugs of abuse. As depicted in Figure 2, the brain's reward center involves the release of the dopamine in the nucleus accumbens, which are nerve cells located underneath the cerebral cortex. Foods and drugs both utilize the dopamine system; the more rewarding the food or drug is evaluated to be, the greater the release of dopamine (Volkow et al., 2008). Thus, an individual addicted to cocaine might experience the same release of dopamine as when an individual eats chocolate cake (Gearhardt, Corbin, \& Brownell, 2009; Volkow et al., 2008). Furthermore, Functional Magnetic Resonance Images (fMRIs) have shown that 
the nucleus accumbens (i.e., the reward center of the brain) is activated for drug users and overeaters when simply looking at pictures of drugs or food, respectively; thus demonstrating that cravings for drugs and foods exist in the same neural pathways (Fortuna, 2012; Wang et al., 2003).

Some theories as to why an inverse relationship might exist between alcohol use and obesity, despite the caloric content of alcohol, is that appetizing foods, drugs, and alcohol might compete for the same reward centers of the brain (i.e. the dopamine system/ nucleus accumbens) (Kleiner et al., 2004; Pickering et al., 2011; Gearhardt, Harrison, \& McKee 2012). Specifically, when the brain's dopamine system is activated by one substance (e.g., food), it may become blocked, mitigating the desire for another substance (e.g., alcohol, drugs) (Gearhardt \& Corbin, 2009). However, to date, there is no direct evidence to prove this.

In addition, examining how pharmaceutical drugs that are prescribed to treat alcohol use disorders also offers support for the notion of food addiction. Alcohol and high fat and/or high sugar foods release opioids in the brain. Opiate blockers such as naloxone are often used in the treatment of alcohol dependence by reducing reinforcement and cravings. Naloxone has also been found to reduce the consumption and preference for rewarding foods in normal eaters, people with obesity, and people demonstrating binge eating disorder (Fortuna, 2012; Wang et al., 2003). Thus, this further offers evidence of the similarities of the brain's physiological responses to drugs/alcohol and rewarding food (Fortuna, 2012; Wang et al., 2003). 
Furthermore, significantly fewer neurotransmitters that regulate the brain's reward and pleasure centers, which are known as D2 receptors, have been found in the brains of individuals with obesity and individuals demonstrating problematic drug use and/or gambling behaviors (Fortuna, 2012; Wang et al., 2003). It is theorized that individuals with low levels of dopamine might seek reinforcing activities or substances to increase dopamine levels, further supporting the notion of food addiction (Fortuna, 2012; Wang et al., 2003).

Pre-clinical research also shows biological support for the concept of food addiction, specifically pertaining to sugar (Coulantuoni et al., 2001; Rada, Avena, \& Hoebel, 2005; Volkow et al., 2008). Rada and colleagues (2005) found that rats which were fed a diet consisting of a sucrose solution, gradually increased their sugar consumption from 37 to $112 \mathrm{ml}$ per day, perhaps demonstrating a tolerance effectsimilar to what happens with drug abuse. Removal of sugar has also demonstrated adverse reactions in rats, similar to removal of a drug from humans (Coulantuoni et al., 2001). Specifically, Colantuoni et al. (2001) reported that rats were observed to have withdrawal symptoms - such as a drop in body temperature and agitated and anxious movements. Lastly, fructose has also been found to have physiological properties similar to other drugs. Because this chemical bypasses the insulin-driven satiety system, it can result in biological changes that result in overeating (C. Davis et al., 2011).

Behavioral Research. The decision to eat is not only based on hunger, but also food palatability and environmental cues, which can also be seen in the use of alcohol and drugs (Volkow et al., 2008). Koob and Le Moal (Koob \& Le Moal, 2008) indicated 
that drug users initially use to experience the pleasurable effects of a drug, and then with repeated use, use drugs to relieve negative emotional states. Parylak and colleagues (2011) have posited a similar transition can occur with food consumption, meaning individuals consume food to relieve negative emotional states, similarly to how negative emotional states could trigger drug and alcohol use (Parylak, Koob, \& Zorrilla, 2011).

In order to help assess the notion of "food addiction," the Yale Food Addiction Scale (YFAS) was developed to assess the addictive tendencies towards food (Gearhardt et al., 2009). The YFAS is a 25 item, self-report assessment that was designed in accordance with symptoms of substance dependence according to the DSM. For example, the YFAS assesses for increased tolerance, continued use despite negative consequences, and withdrawal. A "food addiction diagnosis" is obtained if the individual reports experiencing three or more symptoms over the past year and demonstrates to meet clinically significant impairment (Gearhardt et al., 2009). 


\section{Young Adulthood}

Theoretical Background. This dissertation focuses on younger bariatric surgery patients, who are representative of the young adult cohort, defined as between the ages of 18-35 years. This section first examines developmental theorists' definition of the young adulthood stage and then reviews the literature on what is known about alcohol use and body weight among young adults.

While the exact age range and exact terminology of the young adult cohort differs as will be further described, there is an overall consensus that young adulthood represents a crucial developmental stage in a person’s life, often marked with important developmental tasks, periods of instability, and important life transitions such as entering the workforce and/or marriage (Arnett, 2000; Erikson, 1968; Levinson, 1978; Levinson, 1986). Using this theoretical framework, one of the aims of the proposed dissertation will be to explore psychosocial changes and challenges among young adult bariatric surgery patients.

Traditional developmental models propose that as individuals enter adolescence and young adulthood, they face identity exploration, exploration of close relationships with peers and intimate partners, and separation from parents (Bergman, 1975; Erikson, 1968). Erik Erikson, one of the most influential and earliest lifespan theorists, did not specifically refer to young adulthood, but instead referred to a prolonged adolescence that often occurs in industrialized nations (Erikson, 1968). He further describes this period as the time "during which the young adult, through free role experimentation, may find a niche in some section of his society” (Erikson, 1968 p. 156). 
The psychologist and lifespan theorist Daniel Levinson described life cycles as a sequence of eras, or periods that have their own biopsychosocial characteristics. He called the era from ages 17 to 45, early adulthood. He describes the era of early adulthood as the period of "greatest energy and abundance and of greatest contradiction and stress” (Levinson, 1986 p. 5). Socially this phase is often a time of establishing a place in society, raising a family, and forming and pursuing aspirations. Many people in the early adulthood era enter into parenthood while balancing a career, incur severe financial obligations, and make important decisions regarding lifestyle, work, family, and marriage (Levinson, 1986).

Jeffrey Arnett (2000), another lifespan theorist, calls the years from 18-25 as the emerging adulthood developmental period and maintains that young adulthood is a "better term applied to the thirties, [when individuals are] still young but are definitely adult in a way the 18-25 year olds are not” (Arnett, 2000, p. 477). The emerging adulthood developmental period derives its theoretical basis from Erikson and Levinson, but considers demographic shifts (e.g., later average age of marriage) that have occurred in the past 20 years, when prior developmental theories were developed (Arnett, 2000). He maintains this period is distinct from adolescence and young adulthood and it is a period of relative independence from social roles and societal expectations. While no longer dependent adolescents, emerging adults often do not have adult responsibilities such as a marriage partner and family, and are free to explore various directions in their careers, love life, and overall life experiences. 
Cultural Considerations. It is important to note that the developmental period of young/emerging adulthood might only be relevant in industrialized nations, and/or in certain cultures where individuals typically marry at older ages (Arnett, 2000). Schelegel and Barry (1991) examined adolescence in over 180 non-Western cultures, and concluded that adolescence is a universal life stage. However the authors found that the period between adolescence and adulthood, defined as “youth," applied to only $20 \%$ of the cultures they studied (Schlegel \& Barry, 1991). In their sample, marriage typically signified adulthood, and marriage generally took place between the ages of 16-20 years old.

Arnett (2000) maintains that young adulthood is a period that only exists in cultures where adult roles and responsibilities are postponed (especially if we are defining marriage as an "adult role"). Thus, the period of young adulthood is most common among industrialized nations, as marriage and parenthood are often delayed until educational goals have been met and career opportunities have been explored (Arnett, 2000). However, caution is warranted when examining young adulthood by country, as it is better examined within a cultural context. For example, in the United States, cultural subgroups might promote early parenthood and/or large families (e.g. Mormons). Furthermore, fewer educational opportunities might be available for individuals of lower socio-economic status, and varying cultures might value parenthood over education, making it unlikely for these individuals to treat their young adulthood years as a period of "exploration." 
While theorists might consider events such as entering into marriage and/or the work force as defining moments of young adulthood, perceptions of American young adults differ. Interestingly, studies report that the transition to adulthood is often viewed as the achievement of individualistic milestones such as becoming financially independent and making independent decisions (Arnett, 2003).

Furthermore, this appears to be similar across cultural groups in the United States. Arnett investigated conceptions of the transition to adulthood among emerging adults who were African American, Hispanic/Latino, Asian, and White. Results reveal similarities across ethnic groups. At least $70 \%$ of all groups endorsed the criterion that reflected independence (i.e., "accept responsibility for the consequences of your actions,” “decide on personal beliefs and values,” "financially independent from parents,” and “establish equal relationship with parents”). Thus, the developmental stage of young adulthood is vastly determined by culture and family context (Arnett, 2003).

\section{Body Weight}

Young adulthood is known as a period that is especially influential for excess weight gain, with the greatest increase of overweight and obesity occurring between age 18 and 29 (Gordon-Laresen et al., 2004). This developmental period is also when young adults generally move out of their home of origin and into a new environment, and make independent lifestyle (i.e. food) choices. Thus, the lifestyle changes that occur in young adulthood make this period particularly influential to excess weight gain. Furthermore, research investigating trends in adulthood obesity reveal that gradual weight gain in the 
early adult years often leads to the development of long-standing adulthood obesity (Strong, 2008).

The emerging adult cohort represents a period when young persons are most at risk for the development of overweight and obesity (Gordon-Laresen et al., 2004). In fact, the most recent estimates reveal that among the overall adult population, young people aged 18 to 29 represented the greatest increase in the prevalence of obesity between 1991 and 1998 (Strong, Parks, Anderson, et al., 2008). Perhaps of most concern, overweight or obese children are likely to become overweight or obese as an adult (Dietz, 1998). Furthermore, obesity related comorbidities are even being seen in childhoodmeaning one does not have to be an obese adult to demonstrate the health effects of obesity (Messiah, 2008). Social and economic consequences of early onset obesity can also result, such as a lower likelihood to marry, lower household income, and lower educational attainment (Gortmaker, Must, Perrin, et al., 1993; Sobal, \& Stunkard 1989). As described earlier, young adulthood is also a unique developmental time - marked by many transitions such as moving away from home for the first time. This is also a period of identity development, and subsequently the development of healthy or unhealthy lifestyle characteristics that are an important indicator of long-term behaviors (Bergman, 1975; Erikson, 1968; Gordon-Laresen et al., 2004).

\section{Alcohol Use}

In the general population, adolescents and young adults are especially vulnerable to binge drinking and abusing alcohol (Brown et al., 2008; Chen, Dufour, \& Yi, 2005; SAMHSA, 2006). Among women, non-Hispanic whites and Hispanic/Latinos between 
the ages of 18 to 34 demonstrate the highest prevalence of binge drinking (Centers for Disease Control and Prevention, 2013), with men typically binge drinking twice as often as women (Centers for Disease Control and Prevention, 2013). Recent data reveal that 87\% of young adults aged 19-28 have tried alcohol, with 36\% consuming five or more drinks in a row at least once in the prior two week period (Johnson et al., 2013). In the bariatric surgery literature, younger age has also been associated with problematic alcohol use following bariatric surgery (King et al., 2012).

Contextual and cognitive factors can be involved in one's decision to consume alcohol (O’Hare, 1990; Wall, Hisnon, \& McKee, 1998). Contextual factors include structural and situational factors such as location, peer group, and occasion (i.e. holiday). Cognitive factors include alcohol expectancies, which are beliefs about positive or negative reinforcements from drinking. Examples of positive alcohol expectancies include beliefs that drinking will enhance one’s personality, confidence, and/or sexual relations (Wall, Hisnon, \& McKee, 1998). Overall, the literature indicates that drinking among young adults is related to social atmospheres and positive expectancies (Hartford \& Grant, 1987, O’Hare, 2001).

Furthermore, certain social atmospheres (i.e., the college environment) have also been associated with alcohol consumption in young adulthood. University campuses have been well documented as risk factors for heavy drinking. In fact college represents the period with the highest prevalence of alcohol use disorders (Grant et al., 2004). The most recent data from the Monitoring the Future survey reveals that $37 \%$ of college students reported consuming five or more drinks in a row at least one time in the prior two week 
period, which can be compared to only $30 \%$ of non-college individuals of the same age (Johnson et al., 2013). Other research found that over 30\% of college students met criteria for alcohol use disorders (Knight et al., 2002). Overall the literature demonstrates that college populations generally consume higher quantities of alcohol compared to their non-college attending counterparts (Gfoerer, Greenblatt, \& Wright, 1997).

\section{Bariatric Surgery}

Bariatric surgery, also referred to as weight loss surgery, has become increasingly popular with the increase in the obesity epidemic. In 1990, approximately 16,000 bariatric surgeries were performed in the United States (Davis, 2009). This figure increased to 140,000 in 2005 , to a current estimate of more than 220,000 surgeries performed in the United States and Canada between 2008 to 2009 (Buchwald et al., 2004; Saber, Elgamal, \& McLeod, 2008; Smith, 2007). The health benefits of bariatric surgery such as the reversal of type 2 diabetes and other cardiometabolic disease risk factors have been well-documented in both adults (Buchwald et al., 2004; de la Cruz-Munoz et al., 2011; Gomez-Meade et al., 2013) and adolescents (Messiah et al., 2013a; Treadwell J.R. \& Schoelles, 2008).

Surgical treatment for weight loss was first introduced in the 1950's when surgeons noticed post-operative weight loss among patients who had portions of their stomachs or small intestines removed (Jaunoo \& Southall, 2009). The first surgical procedure performed specifically for weight loss was in the 1970's (Davis, 2009). While this procedure was effective for weight loss, this type of surgery was often associated with major, life threatening post-surgical complications (Davis, 2009; Jaunoo \& Southall, 
2009). Consequently, over the past twenty years, advancement in technology and medical knowledge has led to the improvement in surgical techniques. The latest data reveals that major complications are reported in $2 \%$ of patients and deaths occur in $0.1 \%$ of patients (Frangou, 2014).

Weight loss surgery helps the patient lose weight due to the malabsorption and/or restrictive absorption of calories that result after surgery. Malabsorptive surgeries limit the amount of calories that are absorbed; either by removal of a portion of the stomach or by having food bypass a large portion of the stomach. Restrictive surgeries, on the other hand, allow the patients to only eat small portions of food and therefore restrict the amount of calories the patient is able to ingest (Buchwald et al., 2004; G. Davis, 2009; Jaunoo \& Southall, 2009). Some types of surgeries involve both restrictive and malabsorptive techniques.

The most common types of bariatric surgery procedures in the United States are the Roux-en-Y gastric bypass surgery (RYGB), the laparoscopic adjustable gastric band (LAGB), and the laparoscopic sleeve gastrectomy (LSG) procedure. All three types are performed laparoscopically. The RYGB procedure involves both the restriction and malabsorption of calories. A small stomach pouch is created (that restricts the amount of food consumed) and food is re-routed to bypass most of the stomach and part of the small intestine, which results in the malabsorption of calories and nutrients. Laparoscopic sleeve gastrectomy (LSG) involves the removal of $60-80 \%$ of the stomach, which results in a smaller stomach, or a sleeve. Unlike the RYGB procedure, the digestive tract is not re-routed. LSG, like the RYGB procedure, involves both malabsorption and 
restriction of calories to promote weight loss. The laparoscopic adjustable band procedure (LAGB) on the other hand, is the least invasive of the surgical techniques, and involves an adjustable band placed around the upper stomach, therefore only restricting the amount of food the patient can consume (de la Cruz-Muñoz et al., 2011).

Both the LSG and RYGB procedures also result in neurohoromonal changes due to the shrinking of the stomach; these neurohoromonal changes also potentially assist in weight loss. As discussed earlier, ghrelin, an appetite-stimulating hormone, is reduced with both the RYGB and LSG procedures (Snyder-Marlow, Taylor, \& Lenhard, 2010). It is also important to note that the RYGB and LSG procedures are also associated with risks of micronutrient deficiencies. As such, patients are advised to take daily supplements including iron and B-12 (Synder-Marlow et al., 2010).

\section{Governing Boards \& Policies}

Several organizations have led to the development of bariatric surgery policies and continue to provide oversight of this procedure in the United States. Specifically, the National Institutes of Health, American Society for Metabolic and Bariatric Surgery, and the Surgical Review Committee continue to oversee the field of bariatric surgery and provide recommendations and best practice guidelines.

National Institutes of Health. In 1985 the National Institutes of Health (NIH) publicly established the health implications of obesity, including an increased risk of cardiovascular disease, type 2 diabetes, high cholesterol and increased mortality (NIH Consensus Development Conference, 1996). Following this, in 1991, NIH published a 
consensus statement with specific guidelines for the treatment of obesity (Pories, Dohm, \& Mansfield, 2010). Specifically, the NIH Consensus statement recommended the surgical treatment of obesity (i.e. bariatric surgery) for individuals who have a Body Mass Index (BMI) of 40 and above, or a BMI of 35-39 with at least one obesity related comorbidity such as diabetes or hypertension. For individuals with BMIs under 35, behavioral and/or pharmaceutical interventions were recommended.

In 1991, the National Institutes of Health Consensus Development Conference on Gastrointestinal Surgery for Severe Obesity along with a multi-disciplinary panel of health professionals developed further guidelines for the surgical treatment of obesity. Their recommendations included:

(1) Patients seeking therapy for severe obesity for the first time should be considered for treatment in a non-surgical program with integrated components of a dietary regimen, appropriate exercise, and behavioral modification and support;

(2) gastric restrictive or bypass procedures could be considered for wellinformed and motivated patients with acceptable operative risks;

(3) patients who are candidates for surgical procedures should be selected carefully after evaluation by a multidisciplinary team with medical, surgical, psychiatric, and nutritional expertise; 
(4) the operation should be performed by a surgeon substantially experienced with the appropriate procedures and working in a clinical setting with adequate support for all aspects of management and assessment; and

(5) lifelong medical surveillance after surgical therapy is a necessity.

The consensus statement also states that patients should thoroughly explore alternate treatment options with their physician before electing surgery, and need to be made aware that "lifelong medical surveillance" is needed if surgery is elected. Recommendations also include postoperative care, nutritional counseling, long-term follow up, and evaluation of psychosocial changes that occur during weight loss. Lastly, the statement also recommends the development of standardized questionnaires and structured interviews to evaluate the patient's expectations about post-surgical experiences (NIH consensus statement: Gastrointestinal surgery for severe obesity.1991). As will be discussed later, the American Society of Metabolic and Bariatric Surgery (ASMBS) recently published an update to the NIH statement.

Centers for Medicare and Medicaid Services. In February 2006 the Center for Medicare and Medicaid services announced coverage of bariatric surgery for both Medicare and Medicaid Beneficiaries. Eligibility criteria included those who have a BMI $\geq 40 \mathrm{~kg} / \mathrm{m}^{2}$ or a BMI $\leq 35 \mathrm{~kg} / \mathrm{m}^{2}$ with at least one obesity related co-morbidity, and prior failed attempts at treating obesity. At first the coverage did not include anyone over age 65. However, the committee later determined that they could not find any reliable evidence why the surgery should not be offered to anyone over age 65, and removed the 
age stipulation in a February 2006 policy coverage statement (Mechanick et al., 2009). The 2006 Medicaid coverage announcement also influenced private insurance coverage of bariatric surgery, and private insurance benefits for bariatric surgery became more comprehensive and legitimized (Kazel, 2004).

In general, and while specifics may vary by insurance companies, bariatric surgery is covered by private insurance companies for individuals with a BMI of 40 $\mathrm{kg} / \mathrm{m}^{2}$ or greater, or a BMI of $35-39 \mathrm{~kg} / \mathrm{m}^{2}$ with an obesity-related illness. Patients must also receive the appropriate clearances before surgery is approved, such as a psychological examination (Mechanick et al., 2009). As discussed earlier, a pre-surgical mental health evaluation is required to identify any potential contradictions to surgery such as active substance use or major psychiatric illness, as well as to identify any potential postoperative challenges that could hinder weight loss (Mechanick et al., 2009).

In 2009, the American Society for Metabolic and Bariatric Surgery (ASMBS) reviewed the 1991 NIH Consensus statement and revealed even more specific guidelines for bariatric surgery. The panel recommended surgery for adolescents (12-18), only if they had a BMI of $40 \mathrm{~kg} / \mathrm{m}^{2}$ or greater with severe or moderate degrees of comorbidities (such as pre-diabetes and diabetes); a BMI of $35-39 \mathrm{~kg} / \mathrm{m}^{2}$ only with very severe degrees of comorbidities (diabetes and severe hypertension) or with two or more comorbidities. Further, bariatric surgery was only recommended for persons aged 65 and older if they had a BMI over $35 \mathrm{~kg} / \mathrm{m}^{2}$ with severe comorbidities, such as a severe degree of diabetes. The panel recommended that having a BMI of less than $32 \mathrm{~kg} / \mathrm{m}^{2}$ did not warrant surgery in any age group, which is consistent with the NIH guidelines (Yermilov et al., 2009). 


\section{Pre-surgical Psychological Evaluation}

The 1991 NIH consensus statement recommends candidates for weight loss surgery to receive an evaluation by a mental health professional. Many surgery programs and insurance companies also require that patients undergo psychological evaluations prior to being cleared for surgery in order to remain in compliance with best clinical practice standards (Fabricatore, Crerand, Wadden, Sarwer, \& Krasucki, 2006). The overarching purpose of the pre-surgical mental health evaluation is to identify any potential contradictions to surgery such as active substance use or major psychiatric illness and identify any potential postoperative challenges that could hinder weight loss (Mechanick et al., 2009). However, the only exclusion criteria are active alcohol or substance use disorder. These broad exclusion criteria can present several concerns considering this population might already be vulnerable for Axis 1 and addictive disorders.

A literature review revealed that there is currently no consensus as to best practice pre-surgical mental health assessments, and no standardization among psychological examinations for pre-operative bariatric populations. While the ASMBS does recommend a "psychosocial-behavioral evaluation, which assesses environmental, familial, and behavioral factors,” (Mechanick et al., 2009 pg. S12), there are no evidenced-based assessments or further guidelines for mental health professionals working with bariatric patients.

Fabricatore and colleagues (2006) distributed surveys to bariatric surgeons for disbursement to their mental health assessment providers. Among the results, the 
majority of pre-surgical assessments, including substance abuse assessments, were clinical interviews (98.5\%), while 68.6\% used standardized symptom inventories, followed by personality or psychopathological tests (63.4\%). Cognitive functioning and “projective” personality tests were also used. Thus, there is much variability in the mental health assessments administered to bariatric surgery candidates.

Going further, significant discrepancies between routine clinical psychiatric assessments and research assessments have been found among bariatric patients (Mitchell \& S., 2010). Thus, this suggests the need to provide comprehensive assessments of bariatric surgery candidates beyond the initial assessment. In other words, patients can receive psychiatric exams not only at the initial assessment, but also at more immediate time points before surgery. Equally important, while this remains unexplored in the literature, it is the opinion of the author that clinicians conducting the pre-operative assessments also discuss strengths and potential post-operative challenges with patients and surgeons. Very often, the pre-surgical evaluation is used as a "rubber stamp,” solely to appease insurance companies who are covering the procedure.

\section{Post-Surgical Issues}

Suboptimal weight-loss. Weight loss surgery is not a success story for everyone. The literature estimates that $20 \%$ to $50 \%$ of patients regain weight within the first two years of surgery (Shah, Simha, \& Garg, 2006), and approximately 10-25\% fail to maintain their weight loss within 10 years of surgery (Sarwer et al., 2009). This can result in health consequences such as the re-occurrence of type 2 diabetes, hypertension, and reduced quality of life (Digirogi et al., 2010). 
Non-adherence to dietary, exercise, and psychological recommendations, including attending post-operative services in these areas are some potential explanations as to why patients may fail to lose or maintain weight loss following bariatric surgery (Peacock \& Zizzi, 2012). Peacock and Zizzi (2012) reported that only 23\% of 380 patients reported attending post-surgical exercise consultation services, $17.4 \%$ reported attending individual exercise counseling sessions, 22.6\% attended individual counseling sessions, and 53.4\% attended group counseling services. Furthermore, bariatric surgery patients have documented a high rate of disordered eating such as loss of control over eating, grazing, and emotional eating. Improving psychosocial functioning post-surgery and providing continued care post-surgery could help maintain weight loss and therefore the improvement (or eradication) of obesity-related comorbidities.

Post-surgical weight-loss and substance use. Considering the previously discussed literature that suggests an inverse relationship between alcohol use and BMI (Kleiner et al., 2004), a literature search was conducted to examine the effects of alcohol and drug use on post-surgical weight loss among patients who had bariatric surgery. Tedesco and colleagues (2013) found that patients with a history of drug or substance abuse (not current use) lost more weight at six months post-surgery than those without a reported history of substance abuse; however, the difference was not statistically significant (Tedesco, Hua, Lohnberg, Bellatorre, \& Eisenberg, 2013) Similarly, Heinberg and Ashton (2010) found that patients with a substance abuse history demonstrated significantly higher weight loss at 6, 9, and 12 months post-surgery $(\mathrm{p}<.05)$ (Heinberg \& Ashton, 2010). The researchers have theorized that substance abuse treatment might have 
helped with post-surgical weight loss. That is, learning about coping strategies, triggers, etc., could potentially assist with post-operative weight control.

Conversely, however Adams and colleagues found that having a pre-surgical alcohol or drug use disorder was related to marginally lower weight loss at 12 and 24 months post-surgery (Adams, Gabriele, Baillie, \& Dubbert, 2012). Going further, Odom and colleagues (2010) reported that post-surgical weight regain was significantly correlated with "someone expressing concern" over the respondent's post-surgical drinking or drug use(Odom et al., 2010). While there was no data to indicate if these patients met DSM criteria for a drug or alcohol use disorder, we can assume that if someone is expressing concern over a person's alcohol and/or drug use, it is very likely the individual is demonstrating current "problematic" alcohol or drug use. Overall, there is very limited investigating the effects of a substance use history on post-operative weight loss and if/how current substance use effects post-operative weight loss. Thus, these are factors that will hopefully be explored in future bariatric studies.

Racial/ethnic disparities. The "typical" bariatric surgery patient can be described as white, female, and covered by private health insurance. However, that demographic appears to be shifting. Pickett-Blakely and colleagues (2012) examined sociodemographic trends in bariatric surgery within the United States. In examining data collected from the Healthcare Cost and Utilization Project from 1998, 2001, 2004, and 2007 the number of racial/ethnic minority patients undergoing weight loss surgery significantly increased over time. Specifically, Hispanic/Latinos represented $6 \%$ of the patient population in 1998, compared to 9\% in 2007; non-Hispanic black patients represented $12 \%$ of the patient population in 1998 compared to 13\% in 2007, and non- 
Hispanic white patients of an "other" racial/ethnic category represented $1 \%$ of the patient population in 1998 compared to 6\% in 2007. Conversely, non-Hispanic whites made up $81 \%$ of the patient population in 1998 . This decreased to $72 \%$ in 2007. (Pickett-Blakely et al., 2012).

Income levels also demonstrated significant changes over time, which can be a result of the 2006 Medicare and Medicaid coverage statement. Bariatric surgery patients with an income lower than $\$ 25,000$ presented with an increase in bariatric surgery utilization (from $5 \%$ to $20 \%$ ), while those with a household income over $\$ 45,000$ represented a decrease in representation—-from 33\% in 1998 to 28\% in 2007(PickettBlakely et al., 2012). The increase in representation among lower income patients is most likely, in part, attributed to the 2006 Medicaid coverage decision (Reedy, 2009).

While racial/ethnic minorities were undergoing bariatric surgery at a higher rate in 2007 than in 1998, minority populations remain underrepresented among those receiving weight loss surgery. Wallace and colleagues (2009) found that obese, older, lower income males of racial/minority status living in rural areas with non-private insurance very rarely received bariatric surgery $(\mathrm{OR}=0.0089)$. This is particularly alarming as the highest obesity rates are found in this minority, rural, and economically disadvantaged population (Pickett-Blakely et al., 2012; Wallace, Young-Xu, Hartley, \& Weeks, 2010). These findings corroborate the extant literature, which suggests that racial/ethnic disparities exist when accessing bariatric surgery (Pickett-Blakely et al., 2012). 
Ethnic and racial disparities also appear to exist when examining postoperative complications. Weller and colleagues (2003) found that Hispanics report postoperative complications at a $77 \%$ higher rate compared to other racial/ethnic groups, when examining all types of bariatric surgery (Weller, Rostani, \& Hannan, 2003). Findings by Turner and colleagues (2011) examined bariatric surgery outcomes from 2005 to 2007. They found African-Americans were more likely to have pulmonary embolisms than Hispanics or Whites (OR 2.81), and a higher risk of post-operative bleeding and renal failure was found in Hispanics; further suggesting disparities in health outcomes among racial/ethnic groups who undergo bariatric surgery (Turner et al., 2011).

In addition, weight loss outcomes from both the RYGB and AGB procedures consistently reveal higher weight loss and better results across time points among nonHispanic whites compared to Hispanics and non-Hispanic blacks. Harvin et al (2008) examined patients two years after undergoing Roux-en-Y bariatric surgery, and found that race was a significant predictor of weight loss at two years, with non-Hispanic whites demonstrating the best results. Consistent with these findings, Buffington and colleagues (2006) found that African-Americans had less weight loss following gastric bypass than non-Hispanic whites. Researchers that specifically examined racial/ethnic weight loss differences following surgery among females, revealed similar results, with AfricanAmerican female patients losing considerably less weight following surgery than Caucasians (Havin, DeLegge, \& Garrow, 2008; Turner et al., 2011).

No information can be found in the literature investigating racial/ethnic disparities in bariatric surgery among young adults. However, limited research has been found that 
investigates racial/ ethnic differences in weight loss surgery outcomes among adolescents (Barry \& Petry, 2009; Messiah et al., 2012). The limited existing research suggests that non-Hispanic white teens lose more weight than Hispanic teens, mirroring the adult population (Barry \& Petry, 2009; De la Cruz-Muñoz et al., 2010).

\section{Conclusion}

This chapter provided an exploration of the literature that explores obesity, the obesity and alcohol use connection, food addiction, young adulthood, and bariatric surgery. In essence, this chapter explored factors that might affect young adults who have bariatric surgery. Furthermore, given the growing ethnic/racial diversity of the United States and ethnic group disparities in the current obesity epidemic, a special emphasis was also placed on examining the literature on the topics of interest among racial/ethnic minority populations living in the United States.

Varying environmental, biological, and socio-cultural factors can all influence obesity. Bariatric surgery has been documented to be the most effective intervention for long-standing weight loss in obese populations (Belle et al., 2007).The young adult developmental stage represents unique challenges and might be a time when individuals are especially prone to alcohol use disorders. Thus young adults who undergo bariatric surgery might be especially prone to alcohol use disorders, as will be further explored in this dissertation. The next section presents a systematic review of all of the published articles that report on alcohol and/or drug use among post-operative bariatric populations, and presents a critique of the reviewed studies including their representation of young adults and racial/ethnic minorities. 


\section{Theoretical Framework}

Three theoretical frameworks informed my research questions to investigate alcohol use among young adult bariatric surgery patients, specifically (1) Addiction transfer, (2) Developmental Systems Theory, and (3) Ecological Theory. First, the concept of addiction transfer, which refers to trading one addiction for another, serves as a hypothesized explanation of why some bariatric surgery patients might develop substance use disorders after surgery. Specially, patients might trade food for another addictive substance (i.e. alcohol) following surgery. Second, an integrated framework using Developmental Systems Theory and Ecological Theory were used to inform the research questions investigating the dynamic relationship between individual characteristics and environment while considering developmental transitions and perspectives that are common in the young adulthood/emerging adulthood developmental stage.

\section{Addiction Transfer}

The concept of addiction transfer, as it relates to bariatric surgery patients, has its theoretical underpinnings in the notion of food addiction which is related to the addictive properties of food. Thus, bariatric surgery patients might trade food for alcohol or drugs after their surgery. Food addiction is receiving increasing support in both the medical and psychology literatures. Neurobiological data offers support for humans’ ability to become addicted to food, just as they are able to become addicted to a drug (Volkow et al., 2013; Wang et al., 2003). This section will describe addiction transfer and provide a historical 
overview of the concept as well as the related concepts of cross-addiction, symptom substitution, and substitute addiction.

The concept of addiction transfer related specifically to weight loss surgery patients can be traced back to 2006, when anecdotal evidence regarding addiction transfer following bariatric surgery was seen in mainstream media. On October 24, 2006 the Oprah Winfrey show aired an entire episode entitled "Suddenly Skinny” which focused solely on addiction following weight loss surgery (Sogg, 2007). The show discussed addiction transfer that could occur when a bariatric patient exchanges compulsive eating for alcohol (Sogg, 2007). The show concluded that as many as $30 \%$ of people who have had gastric bypass struggle with new addictions like alcohol use disorders (Sogg, 2007).

Cross Addiction. Cross addiction is another term closely related to the concept of addiction transfer. Cross addiction first appeared in the bariatric surgery literature in 2010 and is defined as when "one compulsive behavior is exchanged for another compulsive behavior” (McFadden, 2010 p. 164). A specific example could be when a weight loss surgery patient is no longer overeating, but instead is abusing alcohol. Prior to this, Fisher and Roget (2008) describe cross addiction as when individuals “seek multiple addictive sources of reinforcement throughout their lifetime” (Fisher \& Roget, 2008). The authors state that cross addictions may manifest in the use of another substance or behaviors (e.g., compulsive gambling, internet addiction, overeating) (Fisher \& Roget, 2008).

Cross-addiction is a term also commonly used in the pathological gambling literature. Griffiths (1994) describes the potential of overlap of chemical and behavioral 
addictions, and exemplifies cross-addiction as a "substance-abusing gambler" (Griffiths, 1994). Thus in this context, cross-addiction is defined as having two or more addictions, specifically a behavioral and a substance addiction (Griffiths, 1994).

Substitute Addiction. Substitute Addiction is another concept with a similar meaning to addiction transfer and cross-addiction. Sussman and Black (2008) define substitute addiction as "any addictive behavior that serves at least one key function previously achieved by another addictive behavior such as elation, escape, excitement” (Sussman \& Black, 2008). Furthermore, addictive behaviors that might serve as addictions include substance addictions (i.e., mood-altering products like caffeine, nicotine, drugs, or alcohol), and process addictions, that can alter one’s mood (i.e., gambling and sex) (Sussman \& Black, 2008). Also, in 12-step recovery programs such as Alcoholics Anonymous, there is a term called " $13^{\text {th }}$ stepping," which some believe refers to an individual's tendency to replace one's former addiction with a new addiction (Sussman \& Black, 2008).

Symptom Substitution. Finally, symptom substitution, another term similar to addiction transfer, cross addiction, and substitute addiction, refers to substituting one symptom for another. Symptom substitution, a popular concept in the 1950s and 1960s especially among psychodynamic and behavioral clinicians and researchers (Tyron, 2008), referred to an unresolved conflict in one’s psyche that leads to a new symptom (Tyron, 2008). An example could be when a child stops sucking his thumb, but soon afterwards begins wetting the bed. Thus, the discourse among psychodynamic clinicians includes the importance of treating the underlying unconscious issue, rather than just the 
observable behavior, so that the underlying issue does not manifest itself in other ways (Tyron, 2008).

Symptom substitution, in the context of appetitive disorders, seems to originate in social work literature in the 1980s, and appears in the context of substance abuse and eating disorders (Zweben, 1987). Brisman and Siegel (1984) describe crossover of addictions as a "manifestation of symptom substitution," with the main issue being a lack of self-regulation. Furthermore, anecdotal accounts reveal eating disorder specialists frequently seeing clients who reported prior alcoholism or drug abuse, meaning they potentially substituted alcohol/drugs for an eating disorder (Zweben, 1987).

Thus, the use of the concept of addiction transfer, which is closely related to cross addiction, substitute addiction, and symptom substitution, seems to have started off anecdotally, with accounts in the mainstream media, and on the Internet (Sogg, 2007; Sons, 2007; Spencer, 2006). Then in recent years, the term became a source of investigation for researchers and appeared in surgical, nursing, and psychology journals (Drew, 2011; Heinberg, Ashton, \& Coughlin, 2012; Ivezaj, Saules, \& Wiedemann, 2012). While methodological issues can make it difficult to empirically test and validate the concept of addiction transfer (Kazdin, 2000), addiction transfer is becoming a popular hypothesis to explain the increase in substance use disorders among bariatric surgery patients that is being documented in the literature. 


\section{Developmental Systems Theory}

Developmental Systems Theory (DST) is a multifactorial framework that posits the dynamic interplay between individual characteristics and ecological systems provides the basis of behavior and developmental change (Lerner \& Castellino, 2002). DST focuses on the attributes of individuals in relation to contextual/ecological conditions at different points in time (Vimont, 2012).

The developmental levels are considered to range from the biological level, individual or psychological level, social/relational level, and sociocultural level. Furthermore, these levels are interrelated and change within one organization or environment is related to changes on other levels (Lerner \& Castellino, 2002). Thus, when examining alcohol use among young adults, a DST framework would consider developmental transitions of young adulthood, changing environments and peer groups, as well as individual and biological factors.

\section{Ecological Theory}

Brofennbrenner's ecological theory (1979) is closely related to DST and posits that a variety of contexts in an environment influence a person's behavior and development- such as familial, cultural, social, and geographical environments. Based on ecological theory the context surrounding an individual is essential to understanding their behavior, as we are all imbedded in an environment with varying facets constantly interacting (Brofenbrenner, 1979). 
The specific systems are referred to as: the microsystem, mesosystem, exosystem, and macrosystem. The microsystem is the immediate environment of the individual, and includes variables they are directly exposed to such as their family and peer group. Geographic environment is also part of a microsystem. The mesosystem comprises microsystems interacting, such as the interactions of one's peer group and family. The exosystem is an environment which indirectly effects the individual such as societal institutions. Finally, the macrosystem is the larger cultural context based on the values, and traditions of the individual's culture (Brofenbrenner, 1979).

Ecological transitions occur when an individual's position in the environment change due to roles and/or settings (Brofenbrenner, 1979). These transitions usually involve changes at the microsystem and mesosystem levels, and can affect the strength of the systems. In turn, this can affect the individual's health and well-being.

\section{Integrated Developmental Systems and Ecological Theory}

Young adulthood often brings about many transitions, freedoms, opportunities and choices. Within an integrated theoretical approach combining Developmental Systems and Ecological Theory, biology, individual characteristics, microsystems, macrosystems, and social-environmental structures, as well as developmental transitions among the age cohort are all considered.

Since alcohol use is often culturally embedded into the young adult cohort and college environments (Wechsler et al., 1998; Blane, 1979), it is important to consider these developmental systems and environments. In addition, it is important to consider that individuals who undergo bariatric surgery are often presented with additional 
transitions and challenges following surgery, potentially affecting individual and microsystemic levels.

Thus, a theoretical framework integrating addiction transfer with an integrated Developmental Systems and Ecology Theory provide a rationale to explore the many unique factors that might affect alcohol use among young adults who have had bariatric surgery. It is the overarching aim of this research to further elucidate the drinking patterns of young adult, predominantly racial/ethnic minority bariatric surgery patients. Examining the prevalence of alcohol use and risky/problematic alcohol use can inform future research, potentially leading to novel intervention and prevention approaches to help alleviate substance use disorders in post-operative bariatric populations. 


\section{Systematic Review Of The Published Literature On Alcohol And Drug Use Among Post-Operative Bariatric Patients ${ }^{1}$}

To date, there has not been a review of the literature to assess: (a) types and rates of alcohol and illicit drug use in post-WLS populations; (b) correlates of post-surgical alcohol and illicit drug use disorders; and (c) implications for future research. The following section presents the first systematic, comprehensive, and critical review of the literature; the researcher examines published articles reporting on alcohol use, problematic alcohol use, and illicit drug use among post-operative WLS patients, and provides recommendations for future research.

\section{Method}

A systematic review of the literature was conducted in January 2015 using the following electronic databases: Medline, Psych INFO, and Social Work Abstracts. Keyword searches were conducted using Boolean operators “AND” and "OR” to specify or broaden the search. The term "bariatric surgery,” was included with all keywords. Separate searches were conducted using specific keywords including: "alcohol*," "psychosocial,” and "substance,” The wildcard character “*” was used to truncate words to include all forms of the root word.

Articles were included in the review if they were published in a peer-reviewed journal (online and/or print format) and addressed: (a) alcohol use and/or (b) illicit drug use among people who had WLS. There was no restriction on publication date or language. Case studies were not included in the review as they are not generalizable to a

\footnotetext{
${ }^{1}$ Note: Parts of this section were previously published and can be referenced at: Spadola, C. E., Wagner, E. F., Dillon, F. R., Trepka, M. J., La Cruz- Munoz, D., \& Messiah, S. E. (2015). Alcohol and Drug Use Among Postoperative Bariatric Patients: A Systematic Review of the Emerging Research and Its Implications..Alcoholism: Clinical and Experimental Research,39 (9), 1582-1601.
} 
broader population. Data were abstracted independently by two of the authors to ensure reliability. Figure 1 details the study selection process.

Figure 1

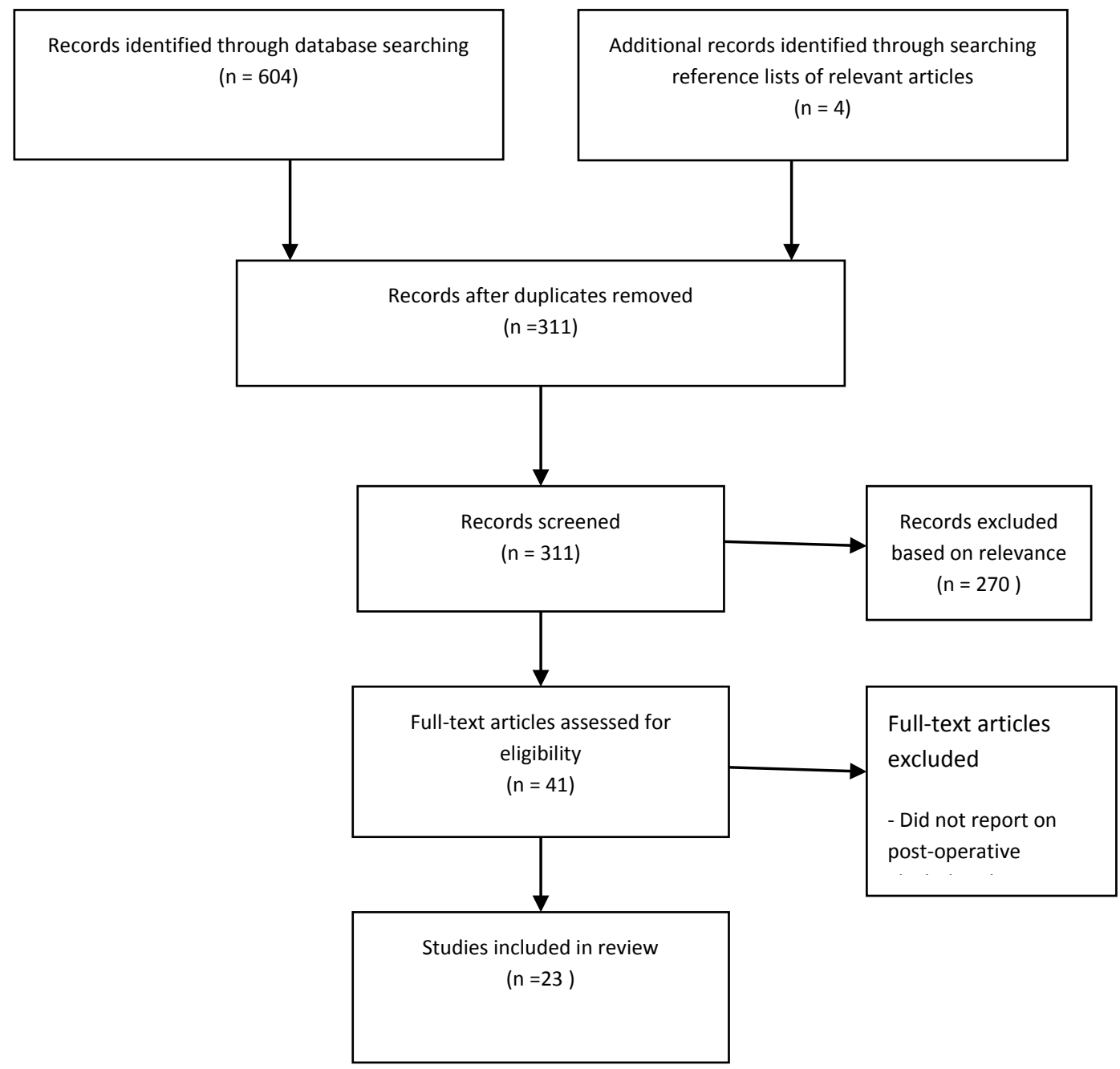

\section{Review}


A total of 23 studies met the search criteria. Nineteen studies reported on alcohol use, and six on illicit drug use (five of these studies also reported on alcohol use). Three studies combined alcohol and drug use into one variable. Overall, study samples were comprised of mainly non-Hispanic white females with mean ages ranging from 40 to 57. Data, including sample demographics and findings regarding alcohol and illicit drug use, were extracted (see Tables 2 and 3 in the Appendix).

\section{Alcohol}

Nineteen studies reported on alcohol use in post-operative WLS patients (Table 2 and 3). Six of these studies used a longitudinal design and assessed patient alcohol use both pre- and post-operatively. Six studies were cross-sectional; pre-surgical alcohol use data (if applicable) was determined through retrospective report or medical charts. Two studies reviewed medical records for alcohol use data. Finally, five studies investigated the prevalence of WLS patients admitted to in-patient treatment for problematic alcohol use.

\section{Longitudinal Studies}

Svensson and colleagues (2013) and King and colleagues (2012) conducted the two most rigorous studies to date, using large sample sizes and prospective, longitudinal designs. Svensson and colleagues (2013) conducted the largest investigation of alcohol use among WLS patients and utilized a non-surgical control group. The authors examined long-term changes in alcohol use risk behaviors and alcohol use disorders at baseline, six months and then 1, 2, 3, 4, 6, 8, 10, 15, and 20 years post-WLS, among Swedish women 
(2,010 WLS patients and 2,037 non-surgical control patients; see Table 2). Overall, the vast majority of respondents (93.1\% of the WLS group and 96\% of the non-surgical group) demonstrated a low prevalence of problematic alcohol use behaviors at all assessment time points.

However, compared to the non-surgical control group, patients who underwent the RYGB procedure were nearly three times as likely to report alcohol use at the medium risk level (defined by the World Health Organization as over 1.5 drinks per day for women), almost five times more likely to have an alcohol abuse diagnosis (as obtained from medical records), and almost six times more likely to report "problems with alcohol” (Table 3; Svensson et al., 2013). Patients who underwent the less-invasive AGB procedure did not demonstrate higher alcohol risk behaviors when compared to the control group. Data concerning alcohol use frequency was collected using a dietary questionnaire. Data concerning problems with alcohol was determined from the question “Do you think you have alcohol problems?” The prevalence of alcohol abuse diagnoses were obtained from medical records (Table 2).

King and colleagues (2012) investigated alcohol use frequency and AUDs at one and two years post-WLS among 1,945 WLS patients; this is the largest investigation of AUDs among WLS patients conducted in the United States. Self-report data collected with the Alcohol Use Disorders Identification Test (AUDIT), an empirically validated and widely used assessment (Allen, Litten, Fertig, \& Babor, 1997), revealed no increase in alcohol use frequency or AUD symptoms within one post-surgical year (Table 3). However, there was a significant increase in AUDs in the second post-surgical year from 
7.6\% to $9.6 \%$ [ $p \leq 0.01]$ ) (King et al., 2012a) . Equally important, RYGB patients were most at risk for AUDs two years post-WLS compared to respondents who underwent the AGB or other surgical procedures (Table 3). Participants were predominantly nonHispanic white females with a median age of 47.

Conason and colleagues (Conason et al., 2012) collected alcohol use frequency data among 155 patients using a questionnaire developed by the study authors (Table 3). While the study had a high attrition rate (i.e., 75\% loss to follow up by 24 months) which may have led to biased findings due to differential dropout, RYGB patients demonstrated a significant increase in alcohol use from pre-WLS to 24 months post-WLS $(p=0.011)$. When examining both RYGB and AGB patients, no significant increase in the frequency of alcohol use was found from pre-WLS to 24 months post-WLS; however, significant increases in alcohol use were found from one month post-WLS to 24 months post-WLS ( $p<0.001$; Conason et al., 2012). Thus, these data could potentially account for patients adhering to instructions to avoid alcohol use for several months following WLS, suggesting increased alcohol use over time.

Conversely, other studies have reported post-surgical decreases in high-risk alcohol use and overall alcohol consumption, (Table 3; Wee et al., 2014; Lent et al., 2013). Specifically, Wee and colleagues(Wee et al., 2014) collected alcohol use data for 541 WLS patients using a shorter, modified version of the AUDIT (AUDIT-C; Dawson et al., 2005; Reinert \& Allen, 2007) (Table 2). Half of RYGB patients and 57\% of AGB patients who reported high-risk alcohol use at baseline no longer reported high-risk alcohol use at one and two years post-WLS (Table 3). However, it is also important to 
note that a subset of respondents who did not report high-risk alcohol use before surgery did report high-risk alcohol use at follow-up, representing "new” post-surgical cases of high-risk alcohol use. This sample was predominantly female (76\%) and non-Hispanic white (69\%) with a mean age of 44 (Wee et al., 2014).

Similarly, Lent and colleagues (Lent et al., 2013a) prospectively examined alcohol use frequency among 155 RYGB patients and found a significant decrease in frequency of alcohol use post-surgery; $72.3 \%(n=112)$ of participants endorsed any alcohol use during the year prior to surgery, which shrank to $63.2 \%$ post-surgery ( $\mathrm{p}=.026)$. Alcohol use data were collected using a non-validated, mailed questionnaire developed by the study authors that was not anonymous. Alcohol use questions are detailed in Table 2 and included: "how often did you have a drink containing alcohol in the past year?” While this study presents important findings regarding a reduction in alcohol use frequency after WLS, due to a lack of methodological rigor (i.e. use of nonvalidated, identifiable, mailed assessments), and the fact that problematic alcohol use was not assessed, implications are limited. The sample was a mean age of 50.1 years ( $\mathrm{SD}=11.3)$, and primarily white (98.1\%), and female (80.6\%).

Alfonsson and colleagues (2014) examined pre- and post-operative problematic alcohol use (as determined by an AUDIT score $>8$ for men and $>6$ for women) among 129 Swedish RYGB patients (Table 2). Compared to pre-WLS, post-operative patients had a lower prevalence of alcohol problems (14\% vs. $5.4 \%$ )(Table 3). Interestingly, however, after surgery three patients met criteria for "alcohol disturbance” (as determined by an AUDIT score over 16), representing new-onset cases. Symptoms of adult ADHD 
were a risk factor for post-surgical problematic alcohol use. Respondents were an average of 43 years old and $78 \%$ female.

\section{Cross-Sectional Studies}

Six cross-sectional studies assessed problematic alcohol use, alcohol use disorders, alcohol sensitivity and/or alcohol use frequency in post-operative WLS patients (Table 2). Overall, while the studies report a low prevalence of problematic alcohol use post-surgery, patients with a history of pre-operative AUDs and those who undergo the RYGB bariatric procedure might be at risk for problematic alcohol use postWLS. However, many of the cross-sectional studies relied on retrospective report of presurgical alcohol use and failed to use validated alcohol use assessment instruments; thus findings must be interpreted within these limitations.

Buffington (Buffington, 2007) conducted an online survey of 318 post-WLS patients (predominantly RYGB) using non-standardized assessments; nearly 30\% reported difficulty controlling their alcohol intake post-surgery compared to only $4.5 \%$ who (retrospectively) reported difficulty pre-surgery (Table 3). Furthermore, 84\% of respondents who drank one or more alcoholic beverages weekly indicated that they were more sensitive to alcohol after surgery than before surgery. Nearly half of the sample was between the ages of 36 and 50, and sample was mostly female (93.7\%). Race/ethnicity was not reported, but the sample included respondents of various nationalities. 
Ertelt and colleagues (Ertelt et al., 2008) investigated alcohol abuse and dependence diagnoses among 70 RYGB patients via a mailed, non-standardized survey based on DSM-IV-TR criteria (Table 2). Prior to surgery, 6 patients (8.6\%) retrospectively met criteria for alcohol abuse or dependence. Post-surgery, the prevalence rate was the same, however 2 patients (2.9\%) newly met the alcohol use disorders criteria post-surgery (Table 3). Moreover, 54.3\% of patients reported responding differently to alcohol post-operatively; 34.3\% $(n=24)$ reported quicker intoxication and $20 \%(n=13)$ reported feeling intoxicated after drinking lower quantities of alcohol. The sample predominantly consisted of non-Hispanic white females and had a mean age of 49 $(\mathrm{SD}=9.2)$ (Table 2).

Mitchell and colleagues (2001) examined AUDs among a sample of 78 RYGB patients via a telephone interview using sections of the SCID (Table 2). Overall, only six patients (7.7\%) met criteria for alcohol abuse or dependence post-surgery, which was assessed 13-15 years after surgery (Mitchell et al., 2001). This sample was predominantly female (83\%), with a mean age of 57 (range 31-77; SD not reported). Race/ethnicity data were not reported.

Macias and Leal (2003) interviewed 140 WLS patients (post-WLS) and analyzed psychosocial differences among patients meeting DSM-IV criteria for Binge Eating Disorder (BED) prior to surgery. The pre-operative BED group $(\mathrm{n}=25)$ was more likely to have symptoms of alcohol dependence post-operatively, based on the Millon Clinical Multiaxial Inventory-II (39.28, SD=22.7 vs. 12.46, SD=18.71; $\mathrm{p}=.001$; Table 3). The sample was primarily female, with the BED group mean age 36.46 ( $\mathrm{SD}=11.72$ ), and non 
BED group 44.61 (SD=9.92). Race/ethnicity data was not reported, but the study was conducted in Spain.

In a sample of 51 WLS patients, Suzuki and colleagues ((Suzuki et al., 2012) collected pre-operative data via medical chart reviews of psychiatric assessments, and post-operative data through telephone interviews using the SCID (Table 2). Thirty-five percent had a history of a lifetime, pre-surgical AUD, and 12\% met criteria for current AUD post-WLS. No patients met criteria for an AUD at time of surgery. Post-surgical AUDs were associated with the RYGB procedure (compared to other surgical procedures) and having a lifetime history of a pre-surgical AUD (Table 3). This sample was predominantly female (86.3\%) with a mean age of 51 (SD=8.7). Race/ethnicity data were not reported.

Finally, Odom and colleagues (Odom et al., 2010) investigated alcohol use frequency and “concern” with alcohol use through mailed, non-standardized surveys to 203 RYGB patients who had WLS over one year ago. Nearly 42\% reported never using alcohol before or after WLS, 20.1\% identified a decrease in post-surgical alcohol use, and 9.1\% reported increased alcohol use post-WLS. Approximately $10 \%$ of respondents reported that someone expressed concern about their alcohol or drug use post-operatively, suggesting problematic alcohol use. Of note, only $24.8 \%$ of the surveys were returned, making the representativeness of the study questionable. In addition, it is not completely clear if the respondents who reported never using alcohol were referring to lifetime use as the response choice was simply "I never drank alcohol before or after [surgery]” (Odom 
et al., 2010). The sample was mainly non-Hispanic white (71.9\%) and female (85\%), with a mean age of $50.6(\mathrm{SD}=9.8)$.

\section{Medical Record Reviews}

Through a review of medical records two studies investigated AUDs among military veterans who had WLS; both studies found a low prevalence of post-operative AUDs. Adams and colleagues(Adams et al., 2012) conducted a chart review of 61 predominantly AGB patients (Table 2) and found that 5 patients (8.2\%) had a presurgical history of alcohol abuse. No patients demonstrated post-surgical AUDs as determined by the AUDIT-C. Tedesco and colleagues (2013) revealed a high prevalence of a pre-surgical DSM-IV-TR-defined alcohol abuse (21.5\%) among a sample of 205 veterans. More than half of this sample was RYGB patients. Six patients demonstrated problematic alcohol use at their follow-up assessment. While patients with a history of alcohol abuse seemed more likely to develop an AUD post-surgery, this did not reach statistical significance. Males in their upper 40s to lower 50s were predominantly represented in both studies. Adams et al. (2012) reported a 70\% non-Hispanic white sample, and Tedesco and colleagues (2013) did not report race/ethnicity data.

\section{The Prevalence of Bariatric Surgery among Patients in Substance Abuse Treatment}

Five studies examined medical records of patients in substance abuse treatment facilities to determine the prevalence of WLS. Because all of these studies reported on treatment admissions for problematic alcohol use, they are reported here. Saules and colleagues (2010) reviewed 7,199 electronic medical records (EMRs) and hard copies of 
medical charts from admissions to a substance abuse treatment facility in Michigan over the course of 3 years. Based on EMR data, 2\% were WLS patients; based on hand searching medical charts, $6 \%$ were WLS patients. Well over half (62.3\%) of the WLS patients were seeking treatment for alcohol use, and 9.4\% were seeking treatment for alcohol as well as an illicit drug.. Findings revealed that $43.4 \%$ of patients did not engage in heavy alcohol or illicit drug use until after surgery, representing new onset cases. Admission to substance abuse treatment occurred an average of 5.4 (S.D.=3.2) years post-surgery.

Wiedemann and colleagues (2013) conducted a follow up to Saules et al. (2010), and reviewed EMRs of inpatient substance abuse admissions from July 2009 to April 2011 (Table 2). Nearly 3\% of 4,658 patients were determined to be WLS patients; the WLS subset was older and more likely to be female and have an alcohol (vs. drug) diagnosis (Table 3). Of this sample, 56 patients participated in a qualitative interview, and the majority of the qualitative interview participants (60\%) developed a "new onset" AUD/ SUD post-surgery (Wiedemann et al., 2013; Table 3). Respondents who participated in the qualitative interview portion of the study were on average 44.8 years old, primarily non-Hispanic white, and predominantly female (Table 3).

Cuellar-Barboza and colleagues (2014) reported that 4.9\% (n=41) of 823 patients in a treatment program for alcohol use problems had undergone the RYGB WLS procedure. Self-reported alcohol consumption patterns as well as clinical impressions and diagnoses, based on DSM-5 criteria, were extracted from EMRs. Nearly $40 \%(\mathrm{n}=16)$ of the 41 RYGB patients also met criteria for an AUD prior to WLS, and 17\% (n=7) did not 
consume any alcohol prior to WLS. Study authors were contacted to elucidate about the remaining 18 patients; this subset reported drinking alcohol prior to WLS, but did not report a prior AUD diagnosis. Thus, 73\% $(n=25)$ of this sample represents "new onset” cases of AUDs that first appeared after WLS. Patients met criteria for an AUD approximately three years post-WLS, and sought treatment an average of 5.4 years post WLS (Table 3). WLS patients were admitted to treatment had a mean age of 46 , and were primarily non-Hispanic white and female (Table 3).

Ostlund and colleagues (2013) conducted largest medical record review to date about the putative association between WLS and substance abuse treatment, using a nationwide patient registry in Sweden from 1980 to 2006. Findings reveal that 11,115 patients underwent WLS, and patients who had the RYGB procedure were more likely to be hospitalized for an AUD than WLS patients who had the AGB or VGB procedures (hazard ratio, 2.3; 95\% CI, 1.7-3.2). The prevalence of treatment admissions for alcohol use was not provided. Participants were on average 40 years old and predominantly female (Table 3). No race/ethnicity data were provided.

Finally, Fogger and colleagues (2012) present a sub-analysis of nurses participating in a monitoring program for nurses with problematic alcohol or illicit drug use. Of 173 respondents, 25 (14\%) were WLS patients. The majority of participants (68\%) indicated that their problem with substances began after they underwent WLS (Fogger, 2012). While frequency statistics of substances used were not reported, the nurses who had WLS were more likely to report problems with alcohol versus illicit drugs (24\% versus 19\%). 


\section{Illicit Drug Use}

Six studies that met review criteria reported on illicit drug use data among postoperative WLS populations (Table 2 and 3). Five of these studies also reported on alcohol use among WLS patients; findings regarding alcohol use were previously described.

\section{Longitudinal Studies}

Conason and colleagues (Conason et al., 2012) represent the only prospective assessment of pre- and post-surgical illicit drug use among post-WLS populations to date. There were no statistically significant differences in illicit drug use before and after WLS (Table 2). However, as described earlier, implications of this study are limited due to high-attrition experienced at follow-up (Table 3).

\section{Cross-Sectional Studies}

Two cross-sectional studies also reported a low prevalence of post-operative substance use disorders (SUDs) in post-operative WLS patients. These findings are summarized in Table 3.

\section{Medical Record Reviews}

Adams and colleagues (Adams et al., 2012) extracted illicit drug use information from medical charts that was prospectively collected pre- and post-WLS at a U.S. Department of Veterans Affairs (VA) facility. Three of 61 patients had a lifetime, presurgical history of cocaine abuse. Post-WLS, one of those 3 patients was using cocaine again, as revealed by urine toxicology screen. Tedesco and colleagues (Tedesco et al., 
2013) also reviewed medical records at a VA facility and found that 48 of 205 patients had a history of a pre-operative SUD. Post-WLS, two patients had a SUD, specifically methamphetamine use.

Finally, Omalu and colleagues (Omalu et al., 2007) reported on death rates and causes of death after WLS in the state of Pennsylvania over nine years. Among the 440 deaths, 14 (3\%) were listed as being attributed to a non-suicidal drug-overdose, most of which occurred at least one year after surgery. While no other details are provided, this suggests an elevated rate of drug overdose deaths compared to the general population (Omalu et al., 2007).

\section{Substance use (alcohol and illicit drug use combined)}

Three studies investigated post-surgical substance use disorders. Because the types of substances were never distinguished (i.e., alcohol, illicit drug, and/or tobacco use), alcohol use and illicit drug use were not separable. Reslan and colleagues (2014) examined 141 RYGB patients (Table 2) for pre- and post-operative "probable substance misuse.” It is important to note that the authors included tobacco, in addition to alcohol and illicit drugs, in their substance use index. The majority of the sample (80\%) reported no substance misuse both before and after WLS. However, of the twenty patients (14\%) who indicated post-surgical substance misuse, $70 \%(n=14)$ did not report pre-operative substance misuse, representing new-onset post-WLS cases. Tobacco, alcohol, and sedatives were the most commonly reported “misused" substances (Reslan et al., 2014). Frequency statistics for each separate substance (i.e., tobacco, alcohol, and illicit drugs) were not indicated. 
Ivezaj and colleagues (2014) reported on a similar patient sample, and found nearly identical rates of probable substance use disorders. The authors used the MAST$A D$ to assess for SUDs and never differentiated between alcohol and illicit drug use (Table 3). Correlates of post-operative SUDs included more years since WLS, more stressful events post-WLS, and more family members with SUD histories (Table 3; Ivezaj et al., 2014). Fowler and colleagues (2014) performed a secondary data analysis on Ivezaj et al.'s (2014) data (Table 2), and found that patients who reported having presurgical problems with foods that were a combination of high in sugar and low in fat, as well as high glycemic index foods (i.e., refined carbohydrates) were at greater risk for developing a "new” onset SUD after surgery (Table 3).

\section{Conclusions}

The most rigorous studies—-those that were prospective, were longitudinal, and involved large samples of WLS patients—reveal that WLS patients who undergo the RYGB procedure are at a heightened risk for post-operative problematic alcohol use compared to (a) non-surgical controls (Svensson et al., 2013), and (b) patients undergoing other WLS procedures (King et al., 2012). In addition, a large-scale medical chart review of 11,115 WLS patients found that RYGB patients were more likely to seek in-patient treatment for AUDs than WLS patients who underwent other types of procedures (hazard ratio, 2.3; 95\% CI, 1.7-3.2; (Ostlund et al., 2013) Findings from two cross-sectional studies (Conason et al., 2012; Suzuki et al., 2012) also found elevated rates of alcohol use problems among RYGB patients. However, the existing literature is far from unequivocal. For example, Wee and colleagues (2014) found that over 70\% of RYGB 
patients reported amelioration in high risk drinking behaviors one year after surgery, compared to only $48 \%$ of AGB patients.

As discussed earlier, physiologic changes that result from the RYGB procedure may offer a putative explanation for an increased vulnerability for post-surgical AUDs (Hagerdorn et al., 2007; Klockhoff et al., 2002; Woodard et al., 2011)Common theories about the causes of these changes reflect anatomy after the gastric bypass, which allows for rapid absorption of alcohol into the blood stream. In fact, RYGB patients have demonstrated blood alcohol content over .08\% after only one drink (Steffen, Engel, Pollert, Li, \& Mitchell, ). To date, this has not been found to be true for AGB patients.

Patient reports of increased alcohol sensitivity following the RYGB procedure are also documented in two of the studies reviewed here (Buffington, 2007; Ertelt et al., 2008). Increased alcohol sensitivity could potentially increase vulnerability for addiction or problematic use (Woodard et al., 2011). Pre-clinical research has also documented increased alcohol consumption in rats that underwent the RYGB procedure (J. F. Davis et al., 2012; Hajnal et al., 2012; Thanos et al., 2012).

For WLS patients overall, and not just RYGB patients, risk factors for postsurgical problematic alcohol use (i.e. high-risk drinking and/or AUDs) include a history of preoperative AUDs (King et al., 2012b; Suzuki et al., 2012), male gender (King et al., 2012a; Svensson et al., 2013) younger age, (King et al., 2012a), any pre-operative alcohol use (Svensson et al., 2013), regular ( $\geq 2$ drinks per week) pre-operative alcohol use, (King et al., 2012a), pre-operative tobacco use (Svensson et al., 2013), pre-operative illicit drug use ((King et al., 2012a), and symptoms of ADHD (Alfonsson et al., 
2014).Three studies also documented that WLS patients might be over-represented in substance use treatment facilities (Saules et al., 2010; Wiedemann et al., 2013; CuellarBarboza), with WLS patients more likely to seek treatment for alcohol use as opposed to illicit drug use (Saules et al., 2010; Wiedemann et al., 2013).

New onset cases of problematic alcohol use. Perhaps some of the most compelling findings from the previously cited studies include the documentation of “new” post-operative AUDs, meaning that WLS patients did not develop symptoms of AUDs until after surgery (Buffington, 2007; Ertelt et al., 2008; Lent et al., 2013b; Wee et al., 2014) While intriguing, the aforementioned finding comes from studies limited by small sample sizes, failure to use validated assessment instruments, and absence of a control comparison group. Also notable is that females over the age of 45 represent the prototypic participant across these studies, a demographic not commonly associated with problematic alcohol or drug use. The National Epidemiologic Survey on Alcohol and Related Conditions (NESARC) found that less than 2\% of women from ages $45-60$ met criteria for an AUD (Grant et al., 2004), and less than 1.6\% of adults aged 45-59 met criteria for an AUD in the past 12 months according to the National Comorbidity Study (NCS-R; Kessler et al., 2005). Thus, It appears that the pro-typical post-WLS patient is remarkably at-risk for AOD problems during a remarkably low risk period in the lifespan.

Alcohol and Illicit Drug Use Disorder Trajectories. Several studies found that post-WLS patients who will have problems with alcohol/substances start experiencing problems a year or more post-surgery. Cuellar-Barboza et al., 2014; Saules et al., 2010; and Wiedemann et al., 2013 documented the significance of one-year post-WLS for 
symptoms of AUDs/SUDs; while, King and colleagues (2012) found the same two years post-WLS (Table 3).

Research Implications. The literature would benefit from the inclusion of younger and racially/ethnically diverse WLS patients in relation to substance use outcomes. While currently, non-Hispanic white females in their mid forties represent the majority of WLS patients (Sudan et al., 2014), younger and racially/ethnically diverse and male bariatric patients warrant inclusion in future research. Improved health outcomes and weight loss have been documented in younger populations (Messiah et al., 2013b; Treadwell J.R. \& Schoelles, 2008) and increases in weight loss have been associated with having WLS at a younger age (Contreras et al., 2013). Equally important, in the general population, younger adults (ages 18 to 35) are at an increased risk for demonstrating problematic alcohol use (Brown et al., 2008; National Institute on Alcohol Abuse and Alcoholism, 2006)' and thus may be at elevated post-surgery risk for substance use problems. Thus, WLS patients in this age range could represent both an emerging population undergoing WLS, and an especially at-risk population for post-surgical alcohol use disorders.

Studies including ethnic/racial minority populations are also warranted. NonHispanic blacks, Hispanics, and Native Americans/American Indians are disproportionately affected by obesity in the United States (Flegal et al., 2012; Schiller et al., 2012). These ethnic/racial populations have an over-representation of candidates for WLS and will likely represent an increasing demographic (Pickett-Blakely et al., 2012). Other than Conason et al. (2012), none of the studies attended to minority populations 
(Table 2). Furthermore, almost $40 \%$ of the studies reviewed did not include race/ethnicity information (Buffington, 2007; Dutta et al., 2006; Mauri et al., 2008; Mitchell et al., 2001; Suzuki et al., 2012; Tedesco et al., 2013; Wendling \& Wudyka, 2011)

The literature also would benefit from longitudinal investigations of postoperative alcohol and illicit drug using reliable and validated measures. Moreover, many studies relied on retrospective report, which is only so accurate. . Prospective studies assessing alcohol and illicit drug use at multiple points post-surgery also would make important contributions to the literature; such studies would help elucidate which and when WLS patients may be at highest risk for AUDs.

It would also be helpful for researchers to routinely obtain specific and separate alcohol and drug use data. Too many studies to date have collected and reported alcohol and drug use data in a single, combined variable. Physiological and psychological responses to alcohol and illicit drugs certainly vary, as do implications of using alcohol compared to illicit drugs. In addition, with the increasing legalization and decriminalization of medical marijuana, the implications of post-operative marijuana use should also be explored (Rummel \& Heinberg, 2014).

The use of validated alcohol use disorder assessments is also warranted. Of the studies reviewed here, only 11 employed validated assessments, most commonly the AUDIT (Table 2). Illicit drug and alcohol use information should also be collected separately from clinical practices and not by treating clinicians to limit responses driven by social desirability (Ambwani et al., 2013). As a final note, many of the studies 
reviewed here relied on now outdated diagnostic criterion to assess alcohol abuse and dependence.

In conclusion, most of the research reviewed in this systematic review, including the most well-designed studies, suggests patients undergoing the RYGB procedure are at risk for post-operative AUDs. Additional research involving longitudinal designs, validated assessment instruments, investigation of large and diverse samples, and follow up after the two-year post-operative point, is warranted. Comparisons of post-operative AUDs across surgery groups is also needed to further elucidate the relationship between type of WLS and post-operative AUDs. 


\section{CHAPTER III: METHOD}

\section{Overview of Mixed Methods Research Design}

The study employed a cross-sectional, mixed methods design to investigate a cohort of predominantly younger adults who had bariatric surgery at the University of Miami Hospital, Center for Bariatric Surgery. Data was derived from (a) quantitative and qualitative data from the parent study conducted at the University of Miami, Miller School of Medicine and (b) primary qualitative data collected by the researcher. The specifics of the parent study and primary data collection will be discussed later in this chapter. As defined by Creswell and colleagues (2003), a mixed methods study: involves the collection or analysis of both quantitative and/or qualitative data in a single study in which the data are collected concurrently or sequentially, are given a priority and involve the integration of the data at one or more stages in the process of research (Creswell, Plano Clark, Gutmann, \& Hanson, 2003, p.212). A mixed method research design allowed the exploration of the complexities that surrounds alcohol use among young adult bariatric surgery patients. Solely employing quantitative data collection and analysis would limit the richness of the data, and only

employing qualitative methods would not generate the prevalence data on alcohol use patterns in this sample (Creswell et al., 2003) 


\section{Sequential Explanatory Mixed Methods Design}

The overall study followed an explanatory sequential mixed method design ${ }^{2}$. This type of mixed methods design involves two phases of data collection, quantitative followed by qualitative data, with the quantitative data typically informing the participants to be purposefully recruited for the qualitative data collection portion. Overall, the aim of a sequential explanatory design is to have the qualitative data further explain the quantitative data (Creswell, 2003; Tashakkori \& Teddle, 2003).

Table 4 details the sequential explanatory design as related to the present study. The first phase of the research involved secondary data analysis of data collected from the parent study. The second phase employed a purposive sampling approach in order to select cases for qualitative data collection. In order to thoroughly explore the experiences and reasons why an increase in alcohol use might result after WLS, the researcher selected patients who reported an increase in alcohol use after WLS as the purposive sample. The third phase involved qualitative data collection. Quantitative and qualitative data were first analyzed separately. In addition, quantitative and qualitative data are also reported separately (see Results chapter).

\footnotetext{
${ }^{2}$ Of note, Tashakkori \& Teddle (2003) refer to this method as explanatory sequential.
} 
It is important to note that there is a minor deviation from the sequential explanatory mixed method design. The data that was collected for the thematic content analysis that analyzed responses from the open ended question "tell me more about your alcohol use behaviors after WLS," was collected concurrently with the quantitative data as a part of the parent study (see Thematic Analysis section of this chapter) was collected concurrently, as opposed to sequentially. This is also depicted in Table 4.

Table 4.

Mixed Methods Sequential Explanatory Design

\begin{tabular}{|c|c|c|c|}
\hline & $\begin{array}{l}\text { Phase 1: Data Collection \& } \\
\text { Analysis }\end{array}$ & Procedure & Product \\
\hline $\begin{array}{l}\text { Parent } \\
\text { Study }\end{array}$ & $\begin{array}{l}\text { Quantitative \& Qualitative Data } \\
\text { Collection } \\
\text { (Qualitative data only one open- } \\
\text { ended question, "Tell me more } \\
\text { about your alcohol use behaviors } \\
\text { after WLS) }\end{array}$ & $\begin{array}{l}\text { Cross-Sectional; Face to } \\
\text { face interviews occurring } \\
\text { post-surgery, quantitative } \\
\text { data and open-ended } \\
\text { question responses collected } \\
\text { concurrently }(n=69)\end{array}$ & $\begin{array}{l}\text { Numeric data } \\
\text { Text (qualitative) data }\end{array}$ \\
\hline \multirow[t]{3}{*}{$\begin{array}{l}\text { Current } \\
\text { Study }\end{array}$} & $\begin{array}{l}\text { Secondary Data Analysis \& } \\
\text { Interpretation }\end{array}$ & 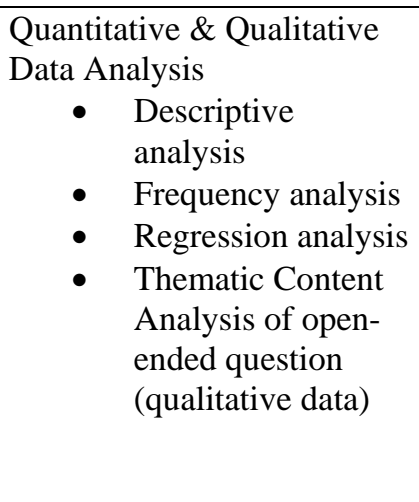 & $\begin{array}{ll}\text { - } & \text { Descriptive } \\
\text { - } & \text { statistics } \\
\text { - } & \text { Frequency statistics } \\
\text { - } & \text { statistics } \\
\text { Major themes from } \\
\text { open-ended } \\
\text { question “Tell me } \\
\text { more about your } \\
\text { alcohol use } \\
\text { behaviors after } \\
\text { WLS” }\end{array}$ \\
\hline & Phase 2: Purposive Sampling & Procedure & Product \\
\hline & Case Selection & $\begin{array}{l}\text { Purposefully select } \\
\text { participants who endorsed } \\
\text { an increase in alcohol use } \\
\text { after WLS, for an in depth- } \\
\text { semi-structured interview. }\end{array}$ & $\begin{array}{l}\text { Eligible participants to be } \\
\text { recruited for qualitative } \\
\text { interviews }\end{array}$ \\
\hline
\end{tabular}




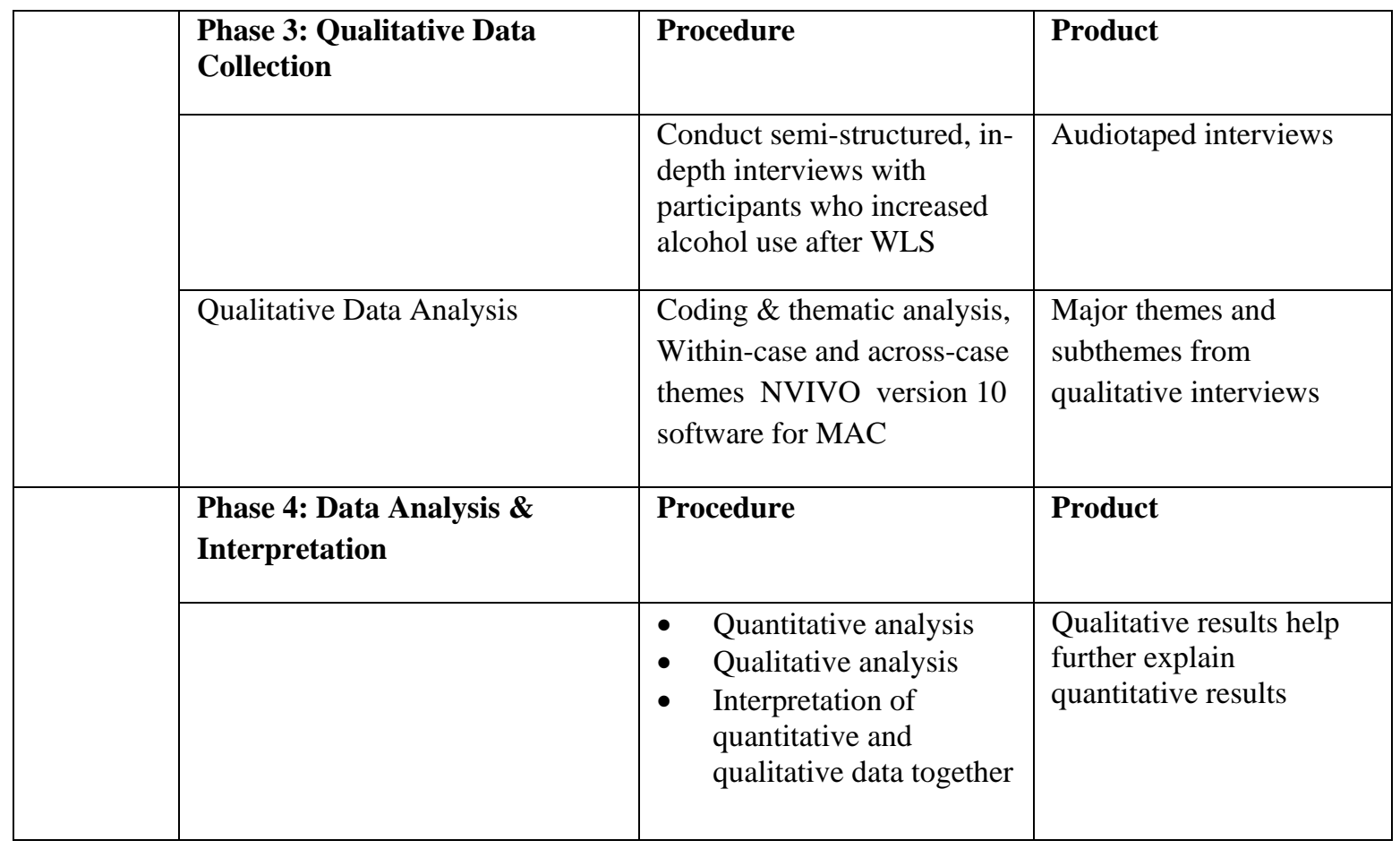

\section{Population}

The research employed quantitative and qualitative data analysis of predominantly racial/ethnic minority young adult (aged 18 to 35) weight loss surgery patients in Miami, Florida. This cohort was selected for several reasons: (1) given that younger populations and racial/ethnic minorities are likely to constitute an emerging demographic undergoing WLS, alcohol use disorders in this population could present an emerging public health concern; (Arnett, 2005) (2) no studies have investigated alcohol use among post-bariatric young adults (Spadola et al., 2015), nor focused on a Hispanic/Latino sample, despite the higher risk of problematic alcohol use among this age group (Brown et al., 2008; Center for Disease Control and Prevention, January 2013); (3) qualitative assessments of phenomenological experiences surrounding post-surgical alcohol consumption are non-existent in the literature. 


\section{Quantitative Data}

Quantitative data was collected under a research study (PI: Messiah) at the University of Miami, Miller School of Medicine, referred to as the “parent study,” which is described in greater detail in the following section. The researcher of the current study was involved in the design and implementation of the parent study, and served as a primary research interviewer.

The parent study involved two cohorts, (1) the retrospective cohort and (2) the prospective cohort. The retrospective cohort was interviewed one time, at least 12 months after their bariatric surgery, and the prospective cohort was interviewed before their surgery and at 5-12 months after their surgery. The data analyzed from the parent study was derived from (1) the retrospective cohort and (2) the (post-operative) follow up interviews of the prospective cohorts.

A convenience sampling approach was implemented for both cohorts. Contact information for participants was extracted from the patient database at the U Health Bariatric Surgery clinic. Eligible participants (under age 35; fluent in the English language; surgery at least 12 months ago for the retrospective cohort; and scheduled to undergo bariatric surgery for the prospective cohort) were initially contacted through a phone call or email. Interested participants were scheduled for an in -person interview with a trained licensed master's or doctoral level clinician. After study procedures were explained, written informed consent was obtained prior to the in-person interview. Participants were paid \$100 for their time, and interviews lasted approximately 3 hours. The University of Miami’s Institutional Review Board (IRB) approved all procedures. 
The total sample ( $\mathrm{n}=69)$ is comprised of patients who were a part of the parent study, specifically 50 participants from the retrospective cohort and 19 from the prospective cohort.

\section{Parent Study}

Secondary quantitative data analysis was performed on data from an existing study at the University of Miami (referred to as the "parent study, "funded by both an institutional and a foundation grant (i.e., University of Miami Specialized Center of Research on Addiction and Health [SCOR; P50DA024584] Developmental Award and the 2011 Micah Batchelor Award for Excellence). The overarching aim of the parent study is to investigate psychosocial and health outcomes among young adults ( $<35$ years old) who have had bariatric surgery, and to investigate the interplay of microbiome hormone (cortisol and serotonin) levels. Specifically, the aims of the parent study include:

a. Determine the pre-and post-surgery prevalence of addictive and/or disordered eating and substance (illicit drugs, nicotine, alcohol) use and abuse.

b. To examine the sex/gender differences in pre-post-surgery prevalence estimates of addiction to food and other substances (illicit drugs, nicotine, alcohol)

c. To explore potential correlates and predicators (e.g. levels of pre-surgery anxiety/depression, impulsivity, etc.) of addiction transfer.

d. To explore potential correlates and predictors (e.g. gut serotonin and cortisol, pre-surgery anxiety/depression, impulsivity, etc.) of addiction transfer.

\section{Contributions of the researcher to the parent study}

The researcher was formerly a project coordinator at the University of Miami, Miller School of Medicine and has significantly contributed to the design, initiation, and 
data collection of the parent study. This included: (1) locating psychometrically sound instruments; (2) providing input on the final assessment battery; (3) contacting authors and /or publishing organizations to obtain measures when necessary; (4) recruiting participants; (5) managing data; and (6) conducting 3-hour long research interviews, including diagnostic assessment of DSM-IV Axis 1 disorders. Data collection procedures in the parent study consist of face-to-face diagnostic interviews (SCID) and face-to-face computer-assisted personal interviews (CASI) to help promote the accurate reporting of sensitive information. 


\section{Quantitative Data Collection Measures}

The following data collection and measurement tools were included in the parent study and selected for analysis in the present study due to their strong psychometric properties and relevance to executing the study aims. The current study analyzed cases of participants that were over 18 years old.

Structured Clinical Interview for DSM Disorders-I-Research Version/Nonpatient Edition (SCID-I-RV/NP). The SCID is a semi-structured interview for making DSM-IV Axis I diagnoses and is known as the gold standard in the field (Spitzer, Williams, Gibbon, \& First, 1990) The SCID is administered as a part of the parent study, to assess lifetime (pre-surgical) and current mental health diagnostic data. Secondary data analysis was performed on the Alcohol Use and Alcohol Dependence modules of the SCID.

Alcohol Use Chart. The Alcohol Use Chart (AUC) is derived from the Alcohol Use Disorders Identification Test (AUDIT), a psychometrically validated instrument (Allen et al., 1997). The AUC asks about age of onset of first alcohol use; past month and past year frequency, quantity, and pattern of alcohol use; consuming alcohol to intoxication, and consuming 4 (if female) or 5 (if male) drinks on one occasion. The AUC also assesses for a self-report of changes in alcohol use and alcohol use behaviors with quantitative and qualitative measures.

\section{Quantitative Data - Management \& Analysis}


All quantitative data analysis was performed using SPSS v. 22, as conducted for the specific aims as delineated below:

\section{AIM 1}

Determine current (past 30 day) frequency of alcohol use as well as alcohol use disorder diagnoses among post-operative young adult bariatric surgery patients. Specifically assess frequency of alcohol use, binge drinking, and frequency of drinking to intoxication, as well as current and lifetime alcohol use disorder diagnoses (assessed using DSM-IV diagnostic schemes).

Frequency statistics, using SPSS v. 22, were used to analyze lifetime and current alcohol use disorder diagnosis as well as frequency of alcohol use, binge drinking, drinking to intoxication, and regular drinking in the past 30 days. As discussed earlier, lifetime and current alcohol use disorder (AUD) diagnosis were assessed using the SCID according to DSM-IV-TR diagnostic criterion, and were entered as dichotomous variables $(0=$ no, $1=$ yes $)$. Current AUD was defined as meeting criteria for an AUD at the time of the parent study interview; lifetime AUD was defined as having a prior (noncurrent) AUD. Alcohol use patterns for current (past current day) alcohol use, drinking to intoxication, and consuming 4 (if female) or 5 (if male) drinks within a few hours of each

other were originally entered as string variables, and were recoded to numerical variables. Response choices were never, monthly or less, 2-4 times per month, 2 times per week, 3 times per week, 4-6 times per week, and daily. In order to quantify the number of “regular" alcohol users (which is defined by the NIAAA as consuming alcohol 3 or more times per week) alcohol frequency data was recoded as a dichotomous variable. The responses “3 times per week,” “4-6 times per week,” and “daily” were combined into one variable indicating regular alcohol use; the remaining response choices “never,” 
“monthly or less," “2-4 times per month,” and "2 times per week” were classified as not meeting criteria for regular alcohol use.

\section{AIM 2:}

Assess psychosocial and physical predictors of past 30 day (post-surgical) frequency of alcohol use, including binge drinking, and drinking to intoxication. Specifically examine if the aforementioned drinking patterns are associated with the following independent variables, informed by the bariatric literature: (a) the RYGB surgical procedure, (b) prevalence of a lifetime (pre-surgical) AUDs, (c) time since surgery, and (d) age.

Three, separate forced entry multiple regression models were run to explore the effect of lifetime alcohol use disorder, age, type of surgical procedure, and time since surgery on (1) number of drinking days in the past 30 days; (2) number of days of drinking to intoxication in the past 30 days; and (3) number of days of binge drinking in the past 30 days. Because all of the independent variables were informed by the bariatric literature, forced entry, as opposed to hierarchical, multiple regression was conducted.

\section{AIM 3:}

$\underline{\text { Analyze self-reported changes in alcohol use and subjective physiological }}$ responses to alcohol after bariatric surgery through quantitative and qualitative methodologies.

Frequency statistics were run to assess the prevalence of participants who stated their alcohol use “increased,” “decreased,” or “remained the same.” Qualitative methodologies also assessed changes in alcohol use following bariatric surgery to meet the goals of AIM 3. The qualitative methods will be discussed in the qualitative results section of this chapter. 
Missing Data. There was no missing data for the quantitative analysis component of the study, most likely because the assessments were administered by a trained interviewer, as opposed to self-administration of the interview by the participant.

\section{Qualitative Data Collection}

Qualitative data was collected through two methods: (1) an open-ended question included in the parent study (i.e., "tell me more about your alcohol use behaviors after WLS”) and (2) semi-structured, in-depth interviews. First the data collection and analysis methods will be described for the open-ended question, followed by the data collection and analysis methods for the semi-structured interview.

Open-ended Question. As described earlier, the open-ended question "tell me more about your alcohol use behaviors after WLS” was included with the parent study. Data collection for the parent study data was previously described. Qualitative data were available for participants who (1) indicated a change in their alcohol use following WLS; and (2) provided a response to interviewers when asked to extrapolate on alcohol use post-WLS (n=29). A content analysis utilizing an inductive, open-coding approach was conducted for responses to this question in order to analyze the major themes that generated from participant responses. A content analysis can be described as a systematic approach to coding and categorizing data in order to determine trends and themes in the content (Hsieh \& Shannon, 2005; Weber, 1990), with the overarching goal of providing "knowledge and understanding of the phenomenon under study" (DowneWamboldt, 1992, p. 314). Another researcher, who was involved with the parent study, reviewed the themes and the participant responses associated with the themes to ensure 
trustworthiness of the data. Six themes surrounding changes in alcohol use post-WLS were determined from the content analysis and are described in the Results chapter.

Semi-Structured, In-Depth Interviews. A purposive sampling approach was employed to obtain in-depth accounts of the phenomenology surrounding individuals who reported an increase in their alcohol use after WLS. As described by Patton (2002), purposeful sampling is utilized when cases for study are selected because they "offer useful manifestations of the phenomenon of interest” (pg. 40). A total of 17 individuals who participated in the parent study indicated increased alcohol use post-WLS, as a part of data collected in the parent study. The researcher contacted all 17 participants through text message, e-mail and or phone call. Of this sample of 17 participants, 12 agreed to an interview; the other 5 participants never responded to the researcher's request for an interview.

Data were collected using an in-depth, semi-structured interview assessment (see Appendix). Interviews lasted between 45 minutes and 1 hour. Participants were paid \$40 in appreciation of their time, and interviews took place at a location of the participant's choosing. Informed consent was obtained prior to the interview. Three interviews took place at office space available to the researcher at the University of Miami, Miller School of Medicine; 4 interviews took place at office space available to the researcher at Florida International University; 4 interviews took place at Starbucks close to the participant's home or workplace; and 1 interview took place at a "tutor" room available at a local library. 
Data saturation was reached after ten interviews, when no new themes were generated from the interviews. However due to the availability of the research participants, the researcher conducted all 12 interviews. Furthermore, it has been determined that 12 is a sufficient number of interviews to provide data saturation in social research (Guest, Bunce, \& Johnson, 2006).

Interviews were digitally recorded and transcribed verbatim and coded by the researcher. Qualitative software, specifically N-VIVO version 10 for Mac, was used to organize and code the transcribed interviews, as well as organize the codes under their appropriate emergent theme. Coding took place in two cycles: (1) initial coding and (2) focused coding. Initial coding is an open-ended approach to examining qualitative data in distinct parts, and comparing data for similarities and differences (Strauss \& Corbin, 1998; Saldana, 2009); the overarching goal of initial coding is to "remain open to all possible theoretical directions indicated by your readings of the data” (Charmaz, 2006, p. 46). Focused coding, after the initial coding of the transcriptions, was employed to determine the most significant categories from the data. Codes were then categorized according to themes, which can be defined as "an implicit topic that organizes a group of repeating ideas” (Auerback \& Silverstein, 2003, p. 38).

\section{Trustworthiness of Data}

Interviewer Training. The researcher who conducted and analyzed the qualitative interviews has extensive qualitative research experience and has been involved in data collection and analysis for federally funded studies. The researcher also has advanced training in qualitative research methods and conducting culturally sensitive research with 
vulnerable populations. Furthermore, the researcher is a licensed clinician and is experienced in establishing rapport.

Internal Checks. In order to ensure the trustworthiness of the data and alleviate bias, several strategies were implemented that have been recommended in the literature (Ezzy, 2002; Saldana, 2009). First, the researcher would often check her interpretations with participants throughout the interview to help ensure interpretative validity (Tashakkori \& Teddle, 2003). For example, she would often employ reflective listening techniques such as "if I heard you correctly, you said ...” and ask participants if her interpretation was correct. Second, the researcher coded as she transcribed her interviews, which can help to capture important details that assist in the accurate interpretation of the data (i.e., the tone of participants, speech patterns). Third, the researcher maintained a reflexive journal that contained her personal impressions of the interviews as well as analytic memos. Since the researcher has been working with WLS patients for several years, this reflexive journal was used to help alleviate any personal biases that she might have, and to ensure her analysis and interpretation of the data were truly inductive.

External Audit. An external audit was also conducted as another step to ensure the trustworthiness of the data (Creswell, 2003; Creswell \& Miller, 2002). The external auditor, a PhD level research scientist who is well versed in qualitative research methodology, reviewed the coded transcriptions and the emergent coding frameworks. Reviewing emergent coding frameworks has been documented in the literature to be a more effective strategy than employing a multiple coding/inter-rater reliability strategy (Barbour, 2001). Specifically, when executing multiple coding strategies, independent coders may reach substantial agreement on codes; however, it is in the development of 
themes and packaging of codes within themes, where considerable variation has been found to exist among researchers (Armstrong, Gosling, Weinman, \& Marteau, 1997; Barbour, 2001). Thus, by conducting an external audit in which an experienced qualitative researcher reviewed the codes and themes, trustworthiness of the data is further promoted. 


\section{CHAPTER IV: RESULTS}

This chapter presents the findings for the quantitative and qualitative data, organized around the research questions. The quantitative data are based on the sample of 69 weight loss surgery (WLS) patients who participated in the parent study, and were interviewed after WLS. Qualitative data are also based on participants who were involved in the parent study, but were recruited though a purposive sampling approach. The quantitative data will be presented first, followed by the qualitative data.

\section{Quantitative Findings}

Demographic Characteristics. Participant demographics and information pertaining to bariatric surgery are presented in Table 5. On average, participants were 26.5 years old $(S D=5.5)$, mostly female $(75 \%)$, and identified as Hispanic/Latino (56.5\%) followed by Non-Hispanic black (27.5\%). The majority of participants had the Roux-en-y-Gastric Bypass procedure (50.7\%) and were interviewed a median of 18 months (range $=5-55$ months) after surgery.

Alcohol Use Behaviors. Alcohol use behaviors were assessed using descriptive statistics in order to assess AIM 1.

Determine current (past 30 day) frequency of alcohol use as well as alcohol use disorder diagnoses among post-operative young adult bariatric surgery patients. Specifically assess frequency of alcohol use, binge drinking, and frequency of drinking to intoxication, as well as current and lifetime alcohol use disorder diagnoses (assessed using DSM-IV diagnostic schemes). 
Current Alcohol Use. As reported in Table 6, 100\% of the sample reported trying alcohol at least one time in their lifetime, with "trying” defined as having more than a sip. In the past 30 days, $66.7 \%$ of the sample used alcohol at least once with $31.9 \%$ consuming alcohol an average of two to four times per month and $17.3 \%$ reporting alcohol use at least 2 times per week. Finally, 8.6\% of the sample reported to be "regular" users of alcohol; that is, consuming alcohol at least three times per week (as defined by NIAAA standards).

Table 5

Demographic Characteristics of the Sample $(N=69)$

\begin{tabular}{ll}
\hline Mean Age (SD) & $26.5(5.5)$ \\
Gender & $75 \%$ female \\
Race/Ethnicity & Hispanic/Latino $56.5 \%$ \\
& Non-Hispanic black 27.5\% \\
& Non-Hispanic white $14.5 \%$ \\
& Non-Hispanic other 1.5\% \\
& \\
Surgery Type & Sleeve $\quad 40.6 \%$ (n=28) \\
& RYGB $\quad 50.7 \%$ (n=35) \\
& Adjustable Gastric Band 8.7\% (n=6) \\
& 18 months (range: 5- 55 months) \\
Median Time Since Surgery* & 25.5 (range =16-36)
\end{tabular}

*Mean= 19.9 months $(\mathrm{SD}, 13)$ 


\begin{tabular}{|c|c|}
\hline $\begin{array}{l}\text { Table } 6 \\
\text { Alcohol Use Behaviors }(N=69)\end{array}$ & \\
\hline Variable & $\mathrm{n}(\%)$ \\
\hline Lifetime Alcohol Use & $69(100 \%)$ \\
\hline Current Alcohol Use Frequency & \\
\hline Daily & $1(1.4 \%)$ \\
\hline 4-6 Times a Week & $1(1.4 \%)$ \\
\hline 3 Times a Week & $4(5.8 \%)$ \\
\hline 2 Times a Week & $6(8.7 \%)$ \\
\hline 2-4 Times per month & $22(31.9 \%)$ \\
\hline Monthly or less & $12(17.4 \%)$ \\
\hline None & $23(33.3 \%)$ \\
\hline Current Drinking to Intoxicatiol & \\
\hline Daily & 0 \\
\hline 4-6 Times a week & $1(1.4 \%)$ \\
\hline 3 Times a week & $2(2.9 \%)$ \\
\hline 2 Times a week & $3(4.3 \%)$ \\
\hline 2-4 Times a month & $12(17.4 \%)$ \\
\hline Monthly or less & $11(15.9 \%)$ \\
\hline None & $40(58.0 \%)$ \\
\hline Current Binge Drinking Freque & \\
\hline Daily & 0 \\
\hline 4-6 Times a week & 0 \\
\hline 3 Times a week & 0 \\
\hline 2 Times a week & 0 \\
\hline 2-4 Times a month & $6(8.7 \%)$ \\
\hline Monthly or less & $4(5.8 \%)$ \\
\hline None & 59 (85.5\%) \\
\hline Lifetime Alcohol Use Disorder & $15(21.7 \%)$ \\
\hline Alcohol Abuse & $15(21.7 \%)$ \\
\hline Pre-operative onset & $12(80 \%)$ \\
\hline Post-operative onset & $3(20 \%)$ \\
\hline Alcohol Dependence & $4(5.8 \%)^{* * *}$ \\
\hline Pre-operative onset & $4(100 \%)$ \\
\hline
\end{tabular}


*Drinking to intoxication defined as "felt the effects of, got a buzz, high, drunk."

** Binge drinking, was defined as consuming 4 (if female) or 5 (if male) within one sitting.

***All 4 participants meeting criteria for alcohol dependence were also included in alcohol abuse frequency.

Drinking to Intoxication. Over half of the sample (58.0\%) reported no current drinking to intoxication, which was defined as drinking until you felt the effects of alcohol, such as getting a buzz, high, or drunk (Table 6). The majority of participants who reported drinking to intoxication did so 2-4 times a month (17.4\%), followed by $15.9 \%$ of the sample who drank to intoxication monthly or less.

Current Binge Drinking. The majority of the sample (85.5\%) reported no current binge drinking, defined as consuming 4 (if female) or 5 (if male) drinks within one sitting. In the past 30 days, 8.7\% reported binge drinking 2-4 times/month, and nearly 6\% reported binge drinking monthly or less.

Lifetime Alcohol Use Disorder. Fifteen participants (21.7\% of the entire sample) had a lifetime alcohol abuse diagnosis; four (5.8\%) of these individuals also met criteria for alcohol dependence. Alcohol abuse and dependence diagnoses were based on administration of the Structured Clinical Interview for DSM-IV TR (SCID). As depicted in Table 6, the majority of participants (80\%) who met criteria for alcohol abuse did so prior to their surgery. Notably, three participants (20\% of the sample meeting criteria for alcohol dependence) met criteria for post-operative alcohol abuse. These participants did not meet criteria for alcohol abuse prior to surgery, demonstrating new onset cases of alcohol abuse that appeared post-operatively. An unanticipated finding is that all 
participants who met criteria for an AUD prior to surgery did not meet criteria for an AUD after surgery, suggesting alleviation of AUDS post-WLS. This will be examined further in the Discussion chapter.

\section{Predictors of Alcohol Use Patterns}

Predictors of Current Alcohol Use Characteristics. Multiple regression analysis was conducted in order to analyze AIM 2. Because all of the independent variables were informed by the bariatric literature, forced entry, as opposed to hierarchical, multiple regression was conducted.

AIM 2: Assess psychosocial and physical predictors of past 30 day (postsurgical) frequency of alcohol use, including binge drinking, and drinking to intoxication. Specifically examine if the aforementioned drinking patterns are associated with the following independent variables, informed by the bariatric literature: (a) the RYGB surgical procedure, (b) prevalence of a lifetime (presurgical) AUDs, (c) time since surgery, and (d) age.

Table 7

Regression Results for Number of Days of Alcohol Use in Past 30 Days

\begin{tabular}{lccc}
\hline Predictor & $b$ & Beta & $p$ \\
\hline Pre-operative Alcohol Use Disorder & 4.48 & .310 & $.012^{*}$ \\
RYGB Surgical Procedure & -.232 & -.021 & .866 \\
Time (Months) since surgery & .020 & .046 & .711 \\
Current Age & .113 & .113 & .356 \\
\hline
\end{tabular}

Note: $p$ value is significant at the .05 level 
The model shown in Table 7 explained $12.6 \%$ of the variance in number of alcohol use days in the past month, $\mathrm{F}(4,64)=2.3, p=.067$ (ns). The most important, and only statistically significant predictor of number of drinking days in the past 30 days was a pre-operative diagnosis of an alcohol use disorder (i.e., alcohol abuse or alcohol dependence based on DSM-IV-TR diagnostic criteria). The diagnosis of a pre-operative AUD was associated with 4.5 more drinking days in the past month, while holding all other predictors constant. Thus, a pre-operative AUD was associated with a higher frequency of drinking days postoperatively.

Predictors of Drinking to Intoxication Frequency. As depicted in table 8, the independent variables explained $6.2 \%$ of the variance in number of days in the past 30 of drinking to intoxication, $F(4,59)=.983$, ns. Thus, having a pre-operative AUD, the RYGB surgical procedure, time since surgery, and current age were not significant predictors of past 30 day drinking to intoxication, which was defined as "felt effects, got a buzz, high, drunk.” 
Table 8

Regression Results for Number of Days of Drinking to Intoxication in Past 30 days

\begin{tabular}{lccc}
\hline Predictor & $b$ & Beta & $p$ \\
\hline Pre-operative Alcohol Use Disorder & 2.25 & .201 & .132 \\
RYGB Surgical Procedure & .34 & 0.04 & .768 \\
Time (Months) since surgery & -.014 & -.044 & .742 \\
Current Age & & & .274 \\
\end{tabular}

Predictors of Binge Drinking Frequency. Pre-operative alcohol use disorder, the RYGB surgical procedure, time since surgery, and age were regressed upon number of days of binge drinking. This model, shown in Table 9, was not a significant predictor of binge drinking, $\mathrm{F}(4,55)=.651$, $n s$. 
Table 9

Regression Results for Number of Days of Binge Drinking in Past 30 days

\begin{tabular}{lccc}
\hline Predictor & $b$ & Beta & $p$ \\
\hline Pre-operative Alcohol Use Disorder & -.271 & -.092 & .506 \\
RYGB Surgical Procedure & .190 & .086 & .541 \\
Time (Months) since surgery & -.014 & -.158 & .254 \\
Current Age & & & .742 \\
\hline
\end{tabular}

Post-Surgical Changes in Alcohol Use. Quantitative and qualitative methodologies assessed changes in alcohol use following bariatric surgery to meet the goals of AIM 3. The quantitative results will be discussed here, and qualitative results will be discussed in the qualitative results section. Frequency statistics, using SPSS v. 21 were used to analyze self-reported changes in alcohol use following WLS. Specifically, participants were asked to quantify if their alcohol use had increased, decreased, or remained the same since WLS.

AIM 3: Analyze self-reported changes in alcohol use and subjective physiological responses to alcohol after bariatric surgery through quantitative and qualitative methodologies. 
Table 10

Frequency Statistics for Change in Alcohol Use Following WLS (N=69)

Behavior Change $n \quad \%$

$\begin{array}{lcc}\text { Decrease } & 26 & 37.7 \% \\ \text { Increase } & 17 & 24.6 \%\end{array}$

$\begin{array}{lll}\text { No Change } & 26 & 37.7 \%\end{array}$

An equal number of participants ( $n=26,37.7 \%)$ reported a decrease or no change in alcohol use behaviors following WLS (Table 10). Conversely, approximately 25\% of the sample reported an increase in alcohol use post-WLS. Themes surrounding changes in alcohol use post-WLS as well as data from in-depth semi-structured interviews will be discussed in the following section.

RYGB Procedure and Increase in Alcohol Use Post-WLS. Contrary to the hypothesis, the RYGB procedure was not associated with an increase in post-operative alcohol use. A binary logistic regression was used to test this hypothesis (see below), and RYGB procedure and increase in alcohol use were recoded to dichotomous variables. The model did not yield statistically significant findings.

Logistic Regression Results for Increase in Alcohol Use

\begin{tabular}{lcrc}
\hline Characteristic & $\begin{array}{r}\text { Odds } \\
\text { Ratio }\end{array}$ & $\begin{array}{r}\text { Standardized } \\
\text { Coefficient }\end{array}$ & $p$ \\
\hline RYGB procedure & .327 & -1.12 & .422
\end{tabular}




\section{Qualitative Findings}

This section reports the qualitative results. The qualitative data are derived from two sources: (1) open ended questions that were asked to participants during the parent study, and (2) semi-structured in-depth interviews that were conducted using a purposive sampling approach.

Thematic Content Analysis of Changes in Alcohol Use Post-WLS. As a part of the parent study, participants were asked if their alcohol use increased, decreased or stayed the same after WLS. Those results, for the entire sample, are reported in the quantitative section of this chapter. Participants were also asked the open-ended question:

“tell me more about your alcohol use behaviors following WLS.” Reported below are the themes derived from thematic content analysis for participants who (1) indicated a change in their alcohol use following WLS and (2) provided a qualitative response to interviewers when asked to extrapolate on alcohol use post-WLS (n=29).

AIM 3: Analyze self-reported changes in alcohol use and subjective physiological $\underline{\text { responses to alcohol after bariatric surgery through quantitative and qualitative }}$ $\underline{\text { methodologies. }}$ 
Table 11

Themes Surrounding Changes in Alcohol Use Following WLS ( $\mathrm{n}=29)$

\begin{tabular}{llll}
\hline & & \multicolumn{2}{c}{ Change in Alcohol Use } \\
Theme & $n^{*}(\%)$ & Increase & Decrease \\
\hline Heightened sensitivity to alcohol & $14(48.3 \%)$ & $4(28.6 \%)$ & $10(71.4 \%)$ \\
Changes in life circumstances** & $7(24.1 \%)$ & $4(57.1 \%)$ & $3(42.9 \%)$ \\
Negative physical effects of alcohol & $3(10.3 \%)$ & 0 & $3(100 \%)$ \\
$* * *$ & & & $3(100 \%)$ \\
Concern for caloric intake & $3(10.3 \%)$ & 0 & 0 \\
Increased socialization & $2(6.9 \%)$ & $2(100 \%)$ & $2(100 \%)$ \\
\hline
\end{tabular}

$* n=31$ because responses fit in more than one category

** Change in marital status/family situation turning legal drinking age.

*** Burning sensation, gas

The thematic content analysis of qualitative data from 29 participants, depicted in

Table 11, revealed six themes surrounding changes in alcohol use post-WLS: (1)

heightened sensitivity to alcohol, (2) changes in life circumstances such as a divorce or marriage, (3) experiencing negative physical effects of alcohol, (4) concern for caloric intake, (5) increased socialization, and (6) concern for overall health. The main theme surrounding changes in alcohol use behaviors was a change in subjective sensitivity to alcohol, which led to a decrease in alcohol use for the majority of participants (71.4\%). 
Concerns for health and caloric intake as well as feeling negative physical effects from alcohol were also attributed to a decrease in alcohol use. Two participants (6.9\%) noticed an increase in socialization following WLS; both of these participants associated this increased socialization with an increase in alcohol use.

\section{Semi-Structured Interviews Investigating an Increase in Alcohol Use Post-}

WLS. In-depth, semi-structured interviews were conducted with 12 participants who participated in the parent study. As detailed in the Methods chapter, participants were recruited via a purposive sampling approach. Inclusion criteria included participants who (a) reported an increase in alcohol consumption following WLS or (b) reported regular ( $\geq 3$ times per week) alcohol use following WLS, as assessed in the parent study. These procedures were carried out in order to meet the goals of aim 4 .

AIM 4: Explore factors surrounding post-operative alcohol use, through semi-structured, in-depth interviews of respondents who reported an increase in alcohol consumption following WLS and/or those who report regular alcohol consumption ( $\geq 3$ times per) post WLS. 


\section{Participant demographics}

Table 12

Qualitative Interview Participant Demographics ( $n=12)$

Mean Age (SD)

Gender

Race/Ethnicity
28.17 (4.5)

83.3\% female

Hispanic/Latino $\quad 58.3 \%$

Non-Hispanic black 33.3\%

Non-Hispanic white $8.3 \%$

Demographics of the sample who participated in the in-depth qualitative interviews are described in Table 12. The majority of participants were females, were a mean age of 28 years old, and were interviewed an average of 35 months, or approximately 3 years, after WLS. The racial/ethnic composition of the sample was Hispanic/Latino (58.3\%), non-Hispanic Black (33.3\%), and non-Hispanic white (8.3\%). All participants reported an increase in alcohol use following WLS; however, for three participants alcohol use both before and after surgery was still infrequent. However, because they all indicated an increase in alcohol use post-WLS compared to before WLS in the parent study, they were included in the in-depth interview component of the study.

The following section reviews the themes as well as the subthemes that emerged from the interviews. Excerpts from participant interviews are also presented, to help illustrate the themes. The four major themes presented include: (1) alcohol as a substitute for food, (2) alcohol use sensitivity, (3) socialization, and (4) alcohol as a coping mechanism. Some quotations are representative of more than one theme; as such they are 
repeated under applicable thematic headings. Themes are further discussed in the

Discussion chapter.

\section{Theme 1: Alcohol as a Substitute for Food}

The first and most compelling theme that emerged from the semi-structured interviews was participants' accounts of replacing food with alcohol. Two sub-themes emerged regarding the substitution of food for alcohol (a): using alcohol as a substitute for food in order to self-soothe and (b): using alcohol as a substitute for food because it's easier to consume.

\section{Subtheme A: Alcohol as a self-soothing mechanism instead of food}

A subset of participants remarked that alcohol was serving as a replacement for food as a self-soothing mechanism. Participants remarked about using alcohol to cope with stress, anxiety, depression, boredom, to assist with sleep, and to also cope with hunger.

I found I can not eat my feelings anymore [after WLS]. I need to find another way to get over my anxiety and depression and stuff like that. It seems like drinking is much easier because you get drunk faster - it goes straight to your liver; it goes down smooth ... It started in August of 2014 when, like, everything started going downhill in my life. I went to food, and I couldn't eat. I could eat like maybe two bites of food and then I couldn't eat any more. I felt that stuffed feeling, "I can't eat any more; I can't eat any more" so I tried to find a way out ...I had a couple of drinks and I had like a little buzz and I felt light headed. Okay, and I could go to sleep. I tried to go to sleep - I took Benadryl but couldn't - so having a few drinks every night is good. Maybe there will be one night a week I don't drink.

$$
\text { -"Laura,” 32-year-old Hispanic/Latina female }
$$

But one of the main differences now is the alcohol intake. It's much higher now than prior to surgery. That's how I soothe myself in the evening. At one point it was vodka. Every single night I had to make a cocktail, now it's more wine ... So that's how I cope. I am more conscientious about what I put in my body because my stomach can only go so far and I throw up.

-“Michelle,” 34-year-old non-Hispanic black female 
[Alcohol] it's kind of like food. I am an emotional eater. When I'm stressed, I eat a lot ... So I do eat but since I know I basically can't down the food, even to this day I can't down it, like eat three hamburgers... I can't eat three hamburgers after surgery [like I could before surgery], but now I can drink four or five shots. .... Because I can't necessarily eat that, and I get that trigger - that pleasure sensation in your head so I think the alcohol substitutes for that.

-“James,” 35-year-old non-Hispanic black male

I was anxious and I was bored so I used to eat.... I would eat junk food. So I felt good, but then afterwards I didn't feel good. But the alcohol doesn't do that. With alcohol, yea, I feel good during the moment.., but I keep feeling good. I may have a hangover, but I don't mind. With the food, I gain weight... I already know food for me was filling in what I didn't have...That supportive family that I wanted. I didn't have the man that was going to be there for me, so that was my excuse. ... Now if I'm bored, I look for other things to do because I know I'm not going to be hungry; I know that I have to be picky of what I eat; I can't eat a lot of things. So I guess that's why I drink more...because maybe I can't eat like what I used to eat; like bread, meat, things like that. I have to be very picky about it because it's not going to go through like it's supposed to..., there are certain foods that I can't eat. So instead of getting frustrated about what I can't eat, I'll go ahead and drink more.

-“Carolina,” 27-year-old Hispanic/Latina female

I used to work and before I left work I had a plan. I knew what restaurant I was stopping by and dessert had to be something very rich and sweet. Then I'd sit on the couch and watch TV... There was always a plan for the food after work, especially if I had a really hard day I would plan to eat something ... with a lot of flavor in it. [Now, post-WLS] I would [drink skinny girl margaritas] every night. I just liked the feeling because I wasn't able to eat as much. So at least I could medicate myself in a way. So that's what I did with the alcohol ... I medicated myself in a way where I wouldn't think about food... It was like I enjoyed this feeling. It's not hunger. I don't have to feed this; I can just relax, fall asleep.

-“Ashley,” 31-year-old non-Hispanic black female

One participant commented that she would often view an online forum about bariatric surgery patients with alcohol problems, which provided validation for her own experience: 
I was like "Oh my goodness, I'm not the only person" so that for sure, before they have the surgery they have to be aware of other habits they may pick up, especially alcohol because it will hinder your weight loss. So that is one of the things they have to be aware of. We are addicts - that's why we became obese. At some point, addicted to food, or emotional eat[ing] is not going away - that habit won't go away. You will look for other habits that fill that void so they have to be extremely careful with that.

-“Ashley,” 31-year-old non-Hispanic black female

Lastly, the following participant was under the impression that alcohol would not contribute to weight gain, which also contributed to her drinking.

I found I was drinking quite a bit. I had to have whatever was at home, whatever wine, I just had to have it. It took the place of hunger. I thought if I didn't eat, I wouldn't gain weight but then again there was a point where I stopped losing. So I had to really look at the numbers because in my mind it was liquid as opposed to solids so I wouldn't gain weight ... .

-“Ashley,” 31-year-old non-Hispanic black female

\section{Subtheme B: Alcohol is easier to consume than food}

The second subtheme that emerged in the context of alcohol serving as a

replacement for food is related to difficulties or frustration with food. For one participant, this theme also overlaps slightly with the theme of using alcohol instead of food to selfsoothe; their quotation is duplicated here.

Well, I hate food. I don't know what to eat when to eat. Food is a lot of suffering man. I'm craving food but I can't eat like I used to...my body won't let me. You know what, I miss eating 10 chicken wings. Now I can only eat 3 or barely 1. I swear, it's like you're a heroin addict and one day your skin is impermeable.

-“Javier,” 27-year-old non-Hispanic black male

When I ate, I would throw up, but when I drank I never threw up. So I was like oh this is a good - not necessarily a healthy alternative, but an alternative to eating.

-"Penelope,” 20-year-old non-Hispanic white female 
I consume more alcohol than I could a meal. So if I'm at a restaurant, I can have 3 beers and if I take a bite of food, I'm full, but I can still keep drinking. Alcohol and food, [they are] completely different.

-“Josefina”, 25-year-old Hispanic/Latina female

So I guess that's why I drink more...because maybe I can't eat like what I used to eat; like bread, meat, things like that. I have to be very picky about it because it's not going to go through like it's supposed to ... there are certain foods that I can't eat. So instead of getting frustrated about what I can't eat, I'll go ahead and drink more.

-“Carolina,” 27-year-old Hispanic/Latina female

\section{Theme 2: Alcohol Use Sensitivity}

Nine of the twelve participants remarked that they were more sensitive to alcohol following surgery. Subthemes that emerged were 1) increased alcohol sensitivity associated with increased drinking motivations and 2) increased alcohol sensitivity associated with decreased drinking motivations. The three participants who did not reveal a change in post WLS sensitivity to alcohol were very infrequent alcohol users both prior to WLS, and also infrequent alcohol users after surgery (albeit they still reported an increase in alcohol use post-WLS). Thus, because they were not frequent alcohol users, it might have been difficult to notice changes in sensitivity. However, the majority of participants reported an increase in alcohol sensitivity following WLS as represented by the following quotes:

Before I may have two, three or four beers - one or two might do it. And I say: "Okay, good, I feel good now.

$$
\text { -“James,” 35-year-old non-Hispanic black male }
$$

I remember I invited some friends over about 2 years ago and we were watching the playoff games and everyone was drinking stuff. I was like "I don't drink that stuff so I'll just have a little wine cooler." I got to just the neck of it, and I was on the couch sleeping. That was a wrap for me. So even with wine now, I can't drink 
it fast and I can't drink more than one at a time. So you can imagine a few weeks ago I had a glass of wine and a few shots on my birthday. Thank God, someone else drove me home.

-“Lucinda,”30-year-old non-Hispanic black female

As discussed earlier, most WLS centers, including the one from which this patient population was recruited from, recommended for WLS patients to not drink for 6 months following surgery. As depicted with the following quote, abstaining from alcohol use might present some particular challenges.

I'm in Vegas for the first time. I'm 21, my friends are 21, why can't I drink alcohol? He [the nutritionist] said it wasn't that I couldn't, it was that my bodyI would get drunk easier. Quicker. He said to be careful; don't go crazy. Don't get those yard drinks; don't get something that's too sugary. Of course, I went to my butt with what he said, and that was the first thing I did. I got a yard drink, a Bahama Mama ... I was so drunk on $1 / 2$ cup.

-“Josefina”, 25-year-old Hispanic/Latina female

\section{Subtheme C: Increased Drinking Motivations}

A subtheme that emerged was that an increased sensitivity to alcohol after WLS was associated with increased drinking motivations. In particular, and as has been documented in the literature, some participants might experience an instant feeling or buzz after drinking alcohol that might contribute to increased motivation to consume alcohol.

I've never gotten high; I've never done drugs, but I assume it works the samethat once you get that high, you're like - "Wow, I want more of that." And if you're doing it recently after surgery, you're going to get that high, because it's going to go straight there because you don't know that feeling. You've never felt it before.

-“Laura,” 32-year-old Hispanic/Latina female

Some participants also remarked on the reduced time it took to feel the effects of 
alcohol:

... And I just liked the feeling. Because I wasn't able to drink as much and I still could feel nice so that was kinda good ... It motivated me to drink more

-“Ashley,” 31-year-old non-Hispanic black female

It seems like drinking is much easier because you get drunk faster - it goes straight to your liver; it goes down smooth...now I get buzzed on two drinks . . . I definitely drink faster, and . . . my inhibitions lower dramatically because I can just get straight there.

-“Laura,” 32-year-old Hispanic/Latina female

One participant indicated that he is drinking more after WLS because he is able to enjoy the feelings of alcohol with less financial strain.

Before I may have two, three or four beers - [now] one or two might do it. And I say, “Okay, good, I feel good now." So therefore, it's less money coming out of the pocket.

-“James,” 35-year-old non-Hispanic black male

\section{Subtheme D: Decreased drinking motivations}

It is important to note that an increased sensitivity to alcohol might also be associated with a decrease in alcohol use. The thematic content analysis, reported previously, found that for the majority of the participants who reported increased sensitivity to alcohol, the majority reported a decrease in alcohol use due to heightened sensitivity to alcohol post-WLS (Table 11). This will be explored further in the Discussion chapter. However, among this purposive sample, of participants who indicated an increase in alcohol use following WLS, only one person attributed heightened alcohol sensitivity to a decrease in their alcohol use. Furthermore, it seems as if a heightened sense of self-esteem also contributed to a decrease in drinking motivations. 
Before, I was able to drink to where I would be able to drink socially. Now I drink one or 2 that's it. I feel drunk. I don't like getting drunk anymore. I got over it. Now [after surgery], I'm [drinking] a lot less. No need to drink. I don't care anymore. Not fun anymore. I mean now I understand why people drink. You have less inhibitions - liquid courage. Now I don't need that --I can go talk to whomever and be social with anyone.

-“Javier,” 27-year-old non-Hispanic black male

\section{Theme 3: Socialization}

An increase in socialization is the third major theme that emerged from the participant interviews. Many participants remarked that following WLS, they were motivated to socialize more. Several participants attributed their increased desire to socialize to a heightened satisfaction with their physical appearance. The following quotes represent that theme.

I felt a lot better. My clothes fit better. I felt better about the way I looked so I wanted to go out because I didn't mind; I wasn't unhappy anymore.

-"Leah,” 23-year-old Hispanic/Latina female

Look, you can wear things that you couldn't wear before. Before I couldn't wear heels, I couldn't wear skirts; so now, why not go out and show it to the world, man?

-“Carolina,” 27-year-old Hispanic/Latina female

So here's the thing. All your life, when you are overweight, you feel like this person when you go out, people are going to look at you and say: "Oh, she should stop eating;" "Oh, she's so ugly; look at her rolls." After surgery when you lose the weight you feel like a completely different person. ...it's an addiction, just like alcohol. It is one of those things that the surgery does to you. It makes you feel better about yourself and do things you wouldn't do before."

-"Laura," 32-year-old Hispanic/Latina female

One participant remarked that she did not experience an increase in socialization after WLS, and attributed it to feeling comfortable about her appearance both before and 
after WLS. As such, this statement further corroborates how socialization and satisfaction with physical appearance might be related.

I have never had a problem with self-esteem. I have [always] been comfortable in my skin. I don't have the greatest body, but I'm healthy enough. You won't see me in a bikini, but I didn't do the surgery for that. But I've never had an issue with going out. [In terms of socialization post-WLS], nothing different; nothing new.

-“Josefina”, 25-year-old Hispanic/Latina female

Another participant remarked that her post-operative weight loss resulted in her being viewed as more approachable, in turn leading to an increase in opportunities to socialize.

[I socialize more now] because people are more opened up to you. Honestly. Sometimes when you are big, you can have a really good resting bitch face but no one is going to want to talk to you if you're 320 pounds. But if you have a bitch face and you're 120 pounds they think "maybe she just has a really bad face" and they will talk to you.

-“Penelope," 20-year-old non-Hispanic white female

Another participant also noticed an increase in approachability post-WLS.

... I mean, I've always had friends; I never had an issue making friends or had issues talking to people. I'm okay going up to someone I don't know and introducing myself. But I guess it was me making that effort, not them. Now I see people coming up to me. I've noticed that. I really don't know what it is, but now it's more people coming to me.

-“Geysell,” 28-year-old Hispanic/Latina female

\section{Subtheme E: Social Environment and Alcohol Use}

It is not uncommon for social functions and events to take place around alcohol, particularly among the young adult cohort. The relationship between socialization and alcohol use was a subtheme that emerged when exploring socialization following WLS, with most participants indicating that an increase in socialization may be associated with 
an increase in alcohol use, which is concurrent with the results reported in the thematic content analysis (Table 11), and will be discussed further in the next chapter.

But yea, in the last year my drinking increased, I guess. A lot of outings take place around alcohol, but I don't think my liking for it has not changed either way.

-“Lucinda,”30-year-old non-Hispanic black female

As one participant attested to, particular work environments (i.e., the restaurant industry) might also promote alcohol use:

Most servers -after we finish our jobs, we all know what is open; Irish Bar is open; the diner is open; there are discounts for servers ... it's just the culture ... we're servers. We serve drinks and then after work, we drink. That's the job; it's always what it was. And then some would have drinks on the job. It's just an alcohol-centric field.

-“Penelope,” 20-year-old non-Hispanic white female

Alcohol use and socialization could be related to the culture surrounding particular alcohol outlets (i.e., bars and clubs). As described by one female participant:

... Because of the fact I would go out and guys would find me attractive and buy me drinks [my alcohol use increased]. One guy came; wanted to buy me a drink; another guy came, bought me a drink, and another. And that actually happened to me about five or six months after surgery. I went out to the club with my friend ... She went home, left me there all alone, and this guy started buying me drinks. If I tell you I remember that night, I don't. He took me home to his house, and I don't remember anything else. So it [WLS] definitely impacted that because people would want to buy you alcohol.

-“Laura,” 32-year-old Hispanic/Latina female

Conversely, yet still corroborating the effect that environmental influence can have on alcohol use, one participant stated that while she experienced an increase in socialization after WLS, she did not experience an increase in alcohol use. Perhaps this is because her socializing would not take place at alcohol outlets; in addition, her peers rarely drank alcohol: 
No [there is no pressure to drink alcohol]. We go to the park or something like that. It's not like going out to party... even when we have our friends over No, rarely [do they drink]. If anything [they will drink], maybe one beer.

-“Taylor,” 26-year-old Hispanic/Latina female

As was described in in the introduction and background chapters, WLS patients are advised to not drink alcohol for six months following surgery to promote post-surgical healing. When asked about any difficulties related to abstaining from alcohol for the first few months after surgery, some participants indicated that avoiding alcohol could be especially difficult in certain environments, further attesting to the influence of environment on alcohol use:

.. if I went out to a club or a party; you know people were drinking. It's not like they were pressuring me to drink because I don't have that problem because I don't try to fit in. But everyone is having fun and laughing and I'm sitting there like "really?" so I tried to avoid drinking as much as possible, but it's just gotten worse. [I would advise to] try to avoid situations where drinking is common. If you're going to go out somewhere, don't go to a club - where everyone is drinking. I think the longer you wait after surgery to drink, the better. It's one of those things. I don't know.

-“Laura,” 32-year-old Hispanic/Latina female

Participants also remarked on age as a factor associated with socialization and alcohol use. When one participant was asked about increased alcohol use after WLS, she replied:

I don't do it because it solves my problems. I don't look at alcohol as a solution. I'd say it's because I'm young; I go out with friends. We go out; we partyBrickell and Flanagan's has $1 / 2$ off beer and pitchers.

-“Josefina”, 25-year-old Hispanic/Latina female 
Furthermore, celebrating rites of passage, such as a $21^{\text {st }}$ birthday, and vacationing in cities associated with heavy alcohol consumption might also result in contexts that can be particularly difficult to navigate:

I remember it was 5 months [after my surgery] they told me I had to wait [to consume alcohol]. And...it was my friend's 21st birthday and we went to Vegas. So, of course, I was in Vegas and I called the doctor and said "I'm in Vegas for the first time. I'm 21, my friends are 21, why can't I drink alcohol?" He said it wasn't that I couldn't, it was that my body - I would get drunk easier. Quicker. He said to be careful; don't go crazy. Don't get those yard drinks; don't get something that's too sugary. Of course, I went to my butt with what he said, and that was the first thing I did. I got a yard drink, a Bahama Mama, and that was the worst experience....I was so drunk on $1 / 2$ cup... Because in my mind, I think I can handle my liquor as I used to when I was heavier.

-“Josefina”, 25-year-old Hispanic/Latina female

Of note, increased socialization following WLS might not be applicable to young adults who have more responsibilities (e.g., childcare, work demands) that preclude them from frequent socialization with peers or visitation of alcohol outlets. As stated by the following 30 year old, female WLS patient:

Me, personally? No [I have not experienced an increase in socialization following WLS] because I work, and with [going to school full time] and the kids I'm not able to. The only time I do go out is when we take them to Disney World. We take them to Disney World twice a year for their birthday but I don't go out.

-“Ashley,” 31-year-old non-Hispanic black female

\section{Theme 4: Alcohol as a coping mechanism}

The fourth and final theme to emerge from the data is using alcohol to cope with psychosocial stress, but not necessarily using alcohol as a replacement for food as was depicted in Theme 1. The quotes here reflect participants' accounts of using alcohol as a coping mechanism, but do not necessarily involve the replacement of alcohol for food: 
I think it's just like the stresses during the day and I think it ties in to why I do it [drink alcohol] at night because it is so stressful during the day. That is my way of winding down...I use it to cope. My drinking is dealing with the issues.

-“Laura,” 32-year-old Hispanic/Latina female

We fell behind on our mortgage ... then we became victims of identity theft and from there we went into foreclosure ... We had to file for bankruptcy so that's why I started to drink - to cope. That's when I [drank vodka for] three months ... A lot.

-“Michelle,” 34-year-old non-Hispanic black female

No sooner did I lose my job than it was [my wife is] pregnant. I was "Oh, great" ... I'd drink a bottle of Moscato wine, and I'm not even a wine drinker. The whole bottle. It was rough for that 11 months ... Therefore, the alcohol, when I'm really stressed, does have that effect. Back at school last year, I had an English paper, and English was always rough for me, I'm a math guy, I bought my first bottle of the Jack Daniels Honey Whiskey. I was like "mmm, this is good." I find myself, the more stressed I became, the more I was willing to try some of the harder liquor. Originally it was a rum coke or Long Island Iced Tea. But that was a social drink. Now I buy a bottle of whiskey. . . I am definitely a stress drinker.

-“James,” 35-year-old non-Hispanic black male

Lastly, one participant remarked how she used alcohol to deal with the slow

weight loss she experienced after WLS.

I got frustrated because gastric bypass surgery wasn't what I expected it to be. I did tons of research but it just wasn't what I was expecting. I had to put in work. It wasn't like "Okay, I'm going to sit here and lose all the weight." I still had to put in work, and I kind of got frustrated because I wasn't losing the weight as fast as my other friends. So I was thinking maybe I did the wrong choice, maybe I'm doing something wrong so then I figured I'd have a glass of wine and think about it. And that just snowballed from there. So that's what actually started it - the fact that I wasn't losing the weight as fast as I wanted to. I. It did make me feel better.

-“Ashley,” 31-year-old non-Hispanic black female

\section{Summary of Results}

Among this sample of 69 predominantly racial/ethnic minority, young adult WLS patients, age, time since surgery, the RYGB procedure, and having a pre-operative 
alcohol use disorder were not predictive of (a) frequency of drinking days , (b) number of days binge drinking, and (c) drinking to intoxication. However, when investigated separately, having a pre-operative AUD was associated with an increased number of current drinking days post-WLS. It is interesting to note that all 12 patients who met criteria for an AUD no longer met criteria for an AUD after WLS; furthermore three patients represented new onset cases of an AUD post WLS.

Nearly one quarter of the entire sample reported an increase in alcohol use after WLS. Analysis of the open-ended question "tell me more about your alcohol use behaviors after WLS” revealed that an increase in socialization after WLS was most attributed to an increase in alcohol use, followed by an increase in alcohol use sensitivity that perhaps also increased drinking motivations.

A purposive sampling approach was conducted to investigate factors surrounding an increase in alcohol use. Semi-structured interviews with 12 participants who reported an increase in alcohol use after WLS revealed 4 major themes concerning alcohol use: (1) alcohol as a substitute for food, (2) alcohol use sensitivity, (3) socialization, and (4) alcohol as a coping mechanism. Consuming alcohol in response to emotional distress was a subtheme that not only emerged in theme 4 , but also theme 1 (but in theme 1 , alcohol was replacing food). 


\section{CHAPTER V: DISCUSSION}

The purpose of this study was to explore the phenomenology and behaviors surrounding alcohol use among young adult, racially/ethnically diverse bariatric surgery patients. To the author's knowledge, this is the first study documenting factors surrounding alcohol use among young adult bariatric surgery patients. While the emerging research suggests that bariatric surgery patients might be at risk for postoperative problematic alcohol use problems (Conason et al., 2012; King et al., 2012; Svensson et al., 2013), there has yet to be an investigation of alcohol use among young adults and/or young adult racial/ethnic minority WLS patients (Spadola et al., 2015).

Furthermore, in spite of the nascent stage of the research, there has yet to be any in-depth, qualitative investigations of bariatric surgery patients as a whole. Investigating post-WLS alcohol use among young adults and young adult racial/ethnic minorities is of particular importance because: (1) the young adult age group (18-35) might be particularly at risk for alcohol use problems (Brown et al., 2008 Centers for Disease Control and Prevention, U.S. Department of Health and Human Services, 2013; Chen \& Kandel, 1995; National Institute on Alcohol Abuse and Alcoholism, 2006); and (2) racial/ethnic minorities are not only disproportionately affected by obesity and already represent an increasing demographic to undergo WLS (Flegal et al., 2012; PickettBlakely et al., 2012), but are more likely to experience negative psychosocial consequences of alcohol use when they do drink (Greenfield, 2001; Mulia et al., 2009; Yoon et al., 2001). Thus, this study employed a sequential explanatory mixed methods design in an effort to gain an in-depth understanding of post-WLS alcohol use behaviors. 
A discussion of the quantitative and qualitative findings will be presented in the next section, followed by implications for practice, policy, and future research.

\section{Quantitative Findings}

Predictors of Alcohol Use Behaviors. The multiple regression results for the main model are contrary to the common findings in the extant literature. Specifically, the regression model that included the following: (a) having a pre-operative alcohol use

disorder, (b) undergoing the RYGB surgical procedure, (c) time since surgery, and (d) current age was not a statistically significant predictor of post-WLS past 30 day alcohol use, binge drinking, or drinking to intoxication. One plausible reason for this is that these patients were interviewed a mean of 20 months after WLS (less than two years). The literature has found that it is not until at least two years after WLS that alcohol use problems begin to manifest (Cuellar-Barboza et al., 2015; King et al., 2012; Ivezaj et al., 2014) or an increase in alcohol use occurs (Conason et al., 2012). Furthermore, of the research investigating inpatient substance abuse admissions among WLS patients, it is not until an average of 5 years after WLS, that WLS patients are admitted for treatment (Saules et al., 2010; Cuellar-Barboza et al., 2015). Perhaps if alcohol use behaviors were assessed in this sample at a greater time point after surgery, some of these predictors would have been significantly associated with post WLS alcohol use behaviors.

When these predictors were regressed upon drinking frequencies separately, having a pre-operative AUD was significantly associated with an increased frequency of current drinking days, post-WLS. Thus, our findings revealed that having a pre-operative AUD is a risk factor for having an increased frequency of drinking days post WLS. It is 
important to note, however, that having an AUD prior to WLS surgery did not constitute a risk factor for having an AUD after WLS. This is described further in the next section.

Alcohol Use Disorders. An interesting quantitative finding concerns the prevalence and duration of alcohol use disorder (AUD) diagnoses in pre and postoperative WLS patients. As described earlier, in order to assess lifetime or current AUD symptomatology, master’s or doctoral level psychology clinicians administered the Structured Clinical Interview for DSM Disorders-I-Research Version/Non-patient Edition (SCID-I-RV/NP), DSM-IV-TR criterion.

A total of 15 participants (21.7\% of the entire sample) met criteria for a lifetime AUD. Twelve participants (80\% of the AUD sample) developed an AUD before WLS; $100 \%$ of these participants no longer met criteria for an AUD after WLS. These data could suggest an alleviation of problematic alcohol use post WLS. This finding is similar to that of Wee and colleagues (2014), who reported that over half of WLS patients who reported "high-risk" drinking prior to surgery, no longer did so at 1 and 2 years postsurgery (Wee et al., 2014). However, it is important to interpret these findings within the context of what was reported earlier having a pre-operative AUD was associated with a higher number of drinking days in the past 30 . Thus, findings suggest that while these participants no longer met criteria for an AUD post-WLS, they were still reporting a higher number of drinking days than the rest of the sample.

Another important finding concerns the etiology of post-WLS AUDs. Three participants, or $20 \%$ of the sample that met criteria for an AUD, developed an AUD postWLS, with no prior history of an AUD. This relatively high prevalence of new-onset 
AUDs after WLS could be especially concerning and suggests that psychosocial or physiological aspects of WLS might be associated with the development of AUDs. A relatively high prevalence of new-onset AUDs has also been documented in the bariatric literature.

Specifically, Ertelt and colleagues (Ertelt et al., 2008) investigated alcohol abuse and dependence diagnoses among 70 RYGB patients via a mailed, non-standardized survey. They found that 2 patients (2.9\%) newly met criteria for alcohol use disorders post-surgery. Saules and colleagues (2010) reviewed 7,199 electronic medical records and found that $43.4 \%$ of patients did not engage in heavy alcohol or illicit drug use until after surgery, representing new onset cases. Finally, Cuellar-Barboza and colleagues (2014) reported that 73\% ( $\mathrm{n}=25)$ of RYGB patients in a treatment program for alcohol use sample represented "new onset” cases of AUDs that first appeared after they had WLS.

Changes in alcohol use post-WLS. Nearly $25 \%(n=17)$ of WLS patients who participated in the parent study reported an increase in alcohol consumption after WLS: 37.7\% $(\mathrm{n}=26)$ reported a decrease, and $37.7 \%(\mathrm{n}=26)$ reported no change. This can be compared to findings reported by Odom and colleagues (2010), with 9.1\% of 203 WLS patients reporting an increase in alcohol use over one year after WLS (see Table 2 and 3; Odom et al., 2010). Thus, the present study has a higher percentage of WLS patients reporting an increase in alcohol use. Perhaps this is in part accounted for by the younger age of the present study---Odom et al.'s sample had a mean age of 50.6 (SD=9.8), compared to a mean age of $26.5(\mathrm{SD}=5.5)$ for the present study. Nonetheless, it is 
important to consider that both studies did not specify differences in frequency and quantity of alcohol use, which could have different implications. For example, some WLS patients might report drinking alcohol more frequently, but in less quantity, which is not captured in the results reported here. These limitations are discussed later in this chapter.

\section{Conclusion for Quantitative Results}

Age, the RYGB procedure, time since surgery, and having a pre-operative alcohol use disorder were not predictive of alcohol use behaviors or problematic alcohol use after WLS, contrary to the existing literature. Perhaps these factors were not found to be significant predictors of problematic alcohol because this sample was interviewed an average of 20 months after WLS. As discussed earlier, the existing research suggests that alcohol use problems do not begin to develop until well after two years post WLS.

Other quantitative findings reveal that having a pre-operative AUD is significantly associated with increased frequency in number of drinking days post-WLS. However, a pre-operative AUD is not associated with having a post-operative AUD; in fact, all 12 participants who had a pre-operative AUD no longer did so after surgery. In investigating the subset of participants who met criteria for an AUD, 20\% ( $\mathrm{n}=3$ ) of cases represented new-onset AUDs, that is, patients did not develop symptoms until after WLS. Lastly, another main finding is that nearly $25 \%$ of the sample reported an increase in alcohol use after WLS. Together, the findings suggest that there are subsets of patients who might be more at risk for problematic alcohol use after WLS, while for another subset of patients - WLS might help to alleviate problematic drinking. 


\section{Qualitative Findings}

The qualitative findings help us to better understand our quantitative results, specifically in terms of exploring: 1) what factors are related to changes in alcohol use after WLS and 2) what factors surround alcohol use phenomenology among a cohort of young adult, predominantly racial/ethnic minority bariatric surgery patients.

Thematic Content Analysis. As part of the parent study protocol, participants were also asked the open-ended question "tell me more about your alcohol use behaviors after WLS.” The major themes surrounding an increase in alcohol use include: (1) a heightened alcohol sensitivity and (2) increased socialization. In particular, heightened alcohol sensitivity could increase vulnerability for addiction or problematic use, due to feeling the effects of alcohol almost instantaneously (Woodard et al., 2011). Overall, considering that a heightened alcohol sensitivity and increased socialization after WLS could be intersecting factors influencing patients’ alcohol use, these findings are particularly compelling. The data from the in-depth qualitative interviews further explores these factors.

Semi-Structured Interviews. Semi-structured interviews were conducted with 12 participants who reported an increase in alcohol use as a part of their responses in the parent study. The major themes surrounding alcohol use were: (1) alcohol as a substitute for food, (2) alcohol use sensitivity, (3) socialization, and (4) alcohol as a coping mechanism.

Alcohol as a substitute for food. Alcohol as a substitute for food, specifically as a self-soothing mechanism, was the most compelling theme that emerged from the 
qualitative data. As was discussed in the literature review and introduction section of the dissertation, the notion of "addiction transfer" or "symptom substitution" has been invoked as an explanation of the findings of the empirical studies that document an increase in alcohol and/or drug use post-WLS. Furthermore, the physiological and anatomical mechanisms of WLS (i.e., a reduced stomach size) do not typically permit patients to consume large amounts of food in a short period of time and a feeling of satiety is reached quicker than before WLS. In addition, overeating after WLS could produce feelings of discomfort that would not appear prior to surgery (Engstrom et al., 2015); moreover, sugary foods can also produce feelings of nausea called "dumping syndrome” (Rusch \& Andris, 2007). Post-operatively patients are instructed to restrict intake of fatty and sugary foods, follow specific diets, as well as eat small, frequent meals (Conceição et al., 2013). Thus, patients who have previously used food to cope with emotional distress might seek other coping mechanisms after WLS. Data from the qualitative interviews reveal that a subset of patients who previously ate in response to emotional distress prior to surgery were no longer able to do so after surgery, and turned to alcohol as a result. As one male participant stated, "I can no longer eat three hamburgers after surgery, but I can drink four or five shots.”

Additionally, it is not uncommon, especially for the first several months after surgery to be especially challenging when a bariatric patient has to rediscover what they are able to eat, and what food their body negatively responds to. Some patients in this sample also reported turning to alcohol in response to difficulties with eating and digesting food (e.g., “When I ate, I would throw up, but when I drank, I never threw up”). 
This theme might especially prominent soon after WLS, when the individual is adjusting to their new dietary restrictions and more likely to experience adverse effects from eating.

Alcohol Use Sensitivity. The second theme that emerged from the qualitative interviews was alcohol use sensitivity. Nine out of 12 participants revealed an increase in alcohol use sensitivity; of note, the 3 patients who did not indicate a change in sensitivity stated they did not have enough pre-WLS experience with alcohol to make a proper comparison. As the reader may recall, alcohol use sensitivity was also depicted in the thematic analysis of the open-ended alcohol use question; those results reveal that increased alcohol use sensitivity led to a decrease in alcohol use for the majority of patients (Table 11).

Conversely, for the 9 participants who revealed an increase in alcohol sensitivity, this was associated with an increase in alcohol use for all but one participant. (It is important to remember a purposive sampling procedure was used to target specifically patients who increased alcohol use after WLS.) Increased alcohol sensitivity could be especially problematic as depicted from the qualitative data. Specifically, even with proper education, WLS patients might not be prepared for their new alcohol tolerance (e.g., "I was so drunk on $1 / 2$ a cup,” and “Thank God someone drove me home.”). In addition, one participant provided testimony to the addiction potential of experiencing instant effects from alcohol (e.g., “Wow I want more of that!”).

Socialization. Many participants reported an increase in socialization following WLS, which constitutes the third major theme that emerged from the semi-structured interviews. A new (post-WLS) satisfaction with physical appearance seemed to provoke 
socialization in a subset of participants: “Look, you can wear things that you couldn’t wear before. Before I couldn’t wear heels, I couldn’t wear skirts; so now, why not and go show the world, man?"

Social environment and alcohol use was a subtheme that emerged. Participants remarked how alcohol surrounds much of their social activities and it can be difficult to not drink in certain social contexts and environments. For example, participants remarked that not drinking at clubs could be difficult, particularly within the first few months after surgery, when WLS patients are strongly recommended to not consume alcohol. One participant, who was a server, remarked on the "alcohol-centric" environment of the restaurant industry. Going further, one female described a situation in which a romantic suitor kept buying her drinks when she was at a club. Specifically, she stated: "One guy came; wanted to buy me a drink; another guy came, bought me a drink, and another . . . If I tell you I remember that night, I don't.” While not explicitly stated by this individual, increased attention from the opposite sex following WLS and the alcohol environment that might often surround dating could also have potential dangers. Thus, taken together, these data attest to the existing research that invoke social atmospheres as being correlated with alcohol use among young adults (Hartford \& Grant, 1987, O’Hare, 2001), and highlight clinical practice implications as will be discussed later.

However, not all participants stated they socialized more frequently after WLS. A 30 year old married woman commented that because of full time work and school demands and having two children, the only time she socialized was “ . . . when we take them to Disney World.” This speaks to the existing literature on the impact that lifestyle 
can have on young adult excessive alcohol consumption, specifically the protective factors of marriage (Duncan, Wilkerson, \& England, 2006; Stone et al., 2012) and children (Power \& Estaugh, 1990).

It is important to take contextual considerations into account when examining the theme of socialization and alcohol use. All research participants, with the exception of one, were residents of Miami-Dade County. Miami-Dade is a well-established, popular tourist location withover 14 million tourists visiting Miami-Dade annually (Sampson, 2014), and the county is also considered a High Intensity Drug Trafficking Area, with high rates of alcohol, marijuana, prescription drug, and club drug use. Moreover, MiamiDade is an international "partying" destination, and the location of several large scale festivals supporting, if not promoting, alcohol use (e.g., South Beach Wine and Food Festival, White Party, Ultra, Aqua Girl, Miami Urban Music and Film Festival, Art Basel, etc.). In addition to these specific festivals, tourism generally and increasingly is associated with frequent or heavy alcohol use. In fact, is it becoming more and more common to travel for the main purpose of consuming alcohol, called "alcotourism." (Bell, 2008; Ornberg \& Room, 2014). Prior research has found that high alcohol use by tourists potentially normalizes a culture of high alcohol use for residents as well (Padilla, 2012). Thus, many of these participants might have been living in an environment in which frequent/heavy alcohol use is normalized.

Alcohol as a Coping Mechanism. The fourth and final theme to emerge regarding participant's alcohol use was alcohol as a coping mechanism. This theme is different than the first theme, in that there is no mention of alcohol as a replacement for food. 
However, it is important to note that realizing one is replacing alcohol for food certainly requires a lot of insight, thus, just because it was not stated by the particular participant does not mean this is the case. Perhaps most notable when exploring this theme is that one participant remarked that she drank alcohol to cope with slow weight loss. This presents important practice and policy implications, as will be discussed later in this chapter.

Since alcohol use is often culturally embedded into the young adult cohort and college environments (Wechsler et al., 1998; Blane, 1979), it is important to consider developmental systems and environments when investigating alcohol use among young adult WLS patients. Specifically, within a theoretical approach combining integrated developmental systems and ecological theory (Brofenbrenner, 1979; Lerner \& Castellino, 2002), individual, micro-systemic, and social-environmental factors, as well as developmental transitions, seem to influence young adult WLS patient alcohol use. Figure 2 highlights the multifactorial nature of young adult problematic alcohol use within an integrated developmental systems and ecological theoretical context. 
Figure 2. Integrated Developmental Systems and Ecological Theoretical Context of Young Adult Problematic Alcohol Use Based on Research Findings

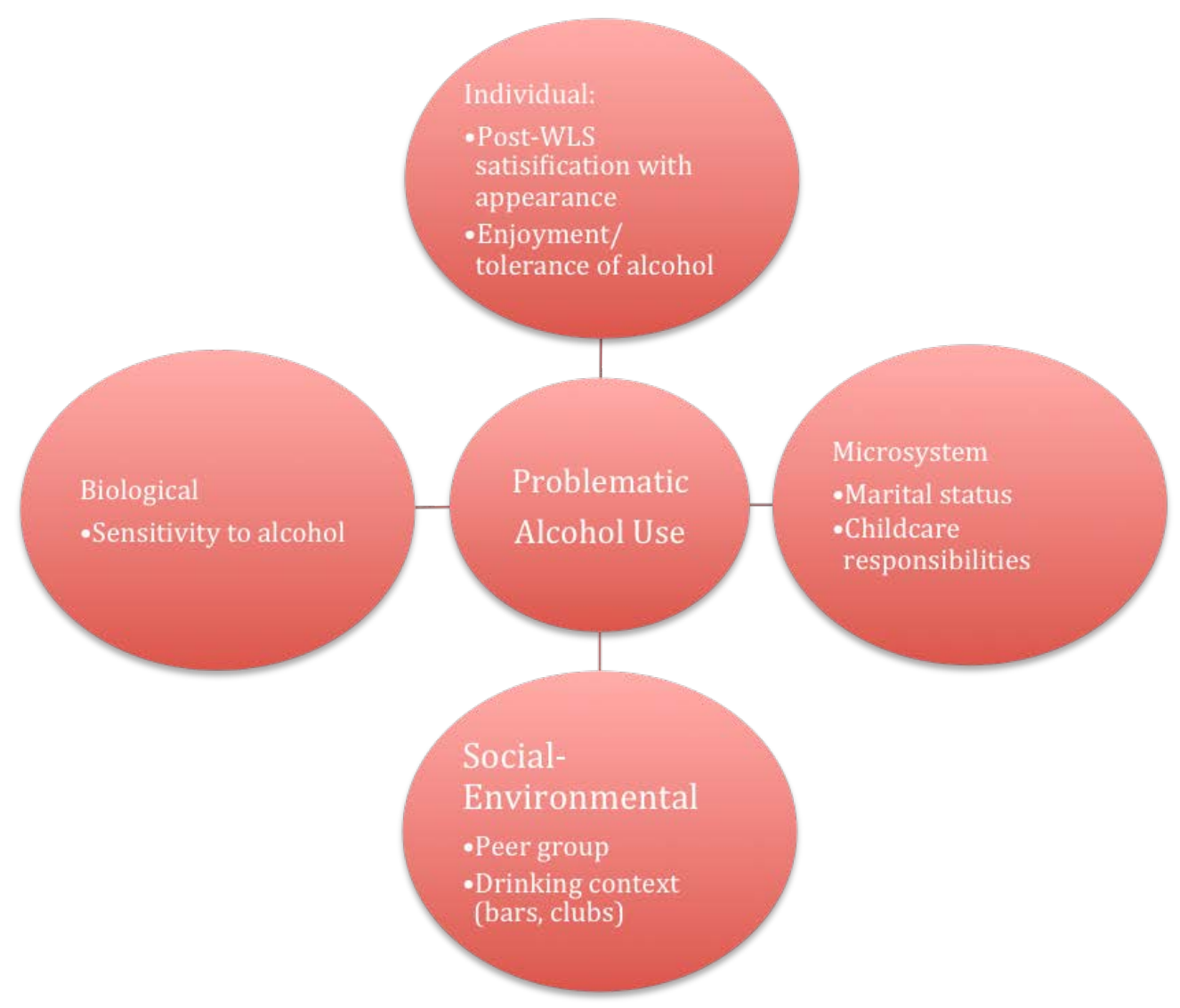




\section{Implications for Clinical Practice}

Within an integrated developmental systems and ecological theoretical approach, the multifactorial contexts and environments that surround young adults should be taken into consideration and pre-operative WLS counseling and education should be tailored to this age cohort. Considering the findings of present study on alcohol use among young adult WLS patients, topics that should be included in WLS education and counseling are: (1) the possibility of trading alcohol for food after WLS, which might be especially salient for individuals who eat in response to emotional distress; (2) healthy coping mechanisms to deal with post-operative frustrations such as negative physical responses to certain foods and slow weight loss; (3) post-WLS sensitivity to alcohol; (4) social environment and its effect on alcohol use.

Furthermore, while our quantitative findings did not determine any potential risk factors for problematic alcohol use (which could be because the sample was interviewed only an average of 20 months after WLS, when most problems with alcohol develop at least 2 years after WLS according to the existing literature), having a pre-operative AUD was associated with an increased number of drinking days after WLS. The existing literature also indicates pre-operative AUDs to be a risk factor for post-WLS problematic alcohol use; thus, WLS patients who have a lifetime history of problematic alcohol need appropriate counseling and education.

Of note, education and counseling of WLS patients should occur before WLS, as it can also assist in the patient's and surgeon's decision regarding if its beneficial to proceed with WLS. Furthermore, while most insurance companies and bariatric surgery facilities in the United States require pre-operative counseling and psychological 
screenings to determine the appropriateness of patients to undergo WLS, post-operative counseling, unfortunately, is voluntary.

Although most bariatric surgery facilities in the United States are required to provide free support groups for WLS patients after surgery, and WLS support groups have been associated with increased weight loss (Orth, Madan, Taddeucci, Coday, \& Tichansky, 2008) research reveals that post-operative WLS support groups are not well attended (Elkins et al., 2005). Research from the parent study (not reported here) indicates that support group attendance is significantly deficient among young adults, perhaps in part because support groups are primarily attended by older individuals, enabling young adults to lack a sense of connection to the group. This makes sense considering demographic trends reveal that the average bariatric patient is in their midforties (Sudan et al., 2014). Thus, going further, having WLS support groups specifically geared towards young adults could potentially promote salubrious post-operative psychosocial outcomes.

Every WLS patient, regardless of age, should continue to be educated on increased alcohol sensitivity that might result after WLS (especially for individuals who had the RYGB procedure which has been shown to especially increase alcohol use sensitivity). Furthermore, interventions also should be tailored to account for participant's developmental stage and context. Specifically for young adults, counseling strategies should involve discussions about socialization plans, drinking at bars or clubs, and alcohol consumption norms of their peer group. Additionally, pre-operative counseling strategies should consider that turning 21 is a milestone for young adults, and frequently could involve heavy alcohol consumption (as was reported in the findings). 
WLS patients need to understand that their increase in alcohol sensitivity could make celebratory drinking occasions especially dangerous.

Equally important, education concerning increased alcohol sensitivity and how it can be associated with increased drinking motivations is also an important topic. In particular, as has been documented in the literature, some participants might experience feeling the effects of alcohol almost instantaneously, which might contribute to motivations to consume alcohol. Thus, educating participants about how feeling an instant "buzz” might lead to increased addiction potential could help to prevent problematic alcohol use.

Education surrounding drunk driving and developing a plan to prevent driving after drinking is also needed. This is especially salient considering that Pepino and colleagues (2015) recently found that among women who had the RYGB procedure, two alcoholic beverages produced a Blood Alcohol Content (BAC) equivalent to four drinks. Thus, education about increased alcohol sensitivity and impaired driving is especially warranted.

Data from this research study also reveals that WLS patients might experience different treatment from others in response to their weight loss. Specifically, the present study indicated that some WLS patients found that they were being asked out more, and/or received more attention from the opposite sex, including being purchased drinks at a club. Considering these social atmospheres coupled with a heightened sensitivity to alcohol, this could lead to situations that make the WLS patient especially vulnerable. Additionally, participants should continue to be educated on post WLS frustrations such as determining what foods their new digestive system can tolerate, and dealing with sub- 
par weight loss. Establishing healthy coping mechanisms for adjustments to new eating patterns and disappointing weight loss prior to WLS could help to alleviate the development of negative coping mechanisms after WLS.

\section{Policy Implications}

From a policy prospective, to ensure that participants are receiving proper education about alcohol use after WLS and the potential challenges that may arise, a standardized educational protocol should be developed and implemented at WLS facilities. This educational protocol should also be tailored to the WLS patient's age group to better account for risk factors that surround their age group.

Of note, participants interviewed for the qualitative portion of the study were asked what they were told about alcohol use after WLS. While many said they remember being told to not drink, other details were vague (such as for how long and why). Thus, providing literature that participants can reference well after WLS surgery may also help to ensure proper dissemination of WLS educational information. Also, at a minimum, WLS facilities should be required to include discussions surrounding alcohol use during pre-operative psychological assessments.

Lastly, in 2013 the American Medical Association classified obesity as a disease (Hurt et al., 2014). While this remains somewhat controversial, this classification will potentially allow for insurance reimbursement for counseling in the treatment of obesity (Hofmann, 2015; Hurt et al., 2014). Thus, hopefully this will also ensure that individual counseling sessions for WLS patients are eligible for insurance reimbursement. Since research indicates that post-operative WLS support group attendance leads to better 
weight loss outcomes (Orth et al., 2008), yet attendance is usually poor (Elkins et al., 2005), offering individual counseling might be a way to ensure WLS patients are obtaining proper mental health treatment after their WLS. Certainly, the findings reported here indicate that WLS involves many post-operative psychosocial adjustments.

\section{Implications for Future Research}

Future research implications include the investigation of younger and racially/ethnically diverse WLS patients in relation to substance use outcomes. While currently, non-Hispanic white females in their mid-forties represent the majority of WLS patients (Sudan et al., 2014), younger and racially/ethnically diverse patients warrant inclusion in future research, as they will likely represent an emerging demographic undergoing WLS. Improved health outcomes and weight loss have been documented in younger populations (Messiah et al., 2013b; Treadwell J.R. \& Schoelles, 2008) and increases in weight loss have been associated with having WLS at a younger age (Contreras et al., 2013). Equally important, in the general population, younger adults (ages 18 to 35) are at an increased risk for demonstrating problematic alcohol use (Brown et al., 2008; National Institute on Alcohol Abuse and Alcoholism, 2006)' and thus may be at elevated post-surgery risk for substance use problems. Thus, WLS patients in this age range could represent both an emerging population undergoing WLS, and an especially at-risk population for post-surgical alcohol use disorders.

Studies including ethnic/racial minority populations are also warranted. NonHispanic blacks, Hispanics, and Native Americans/American Indians are disproportionately affected by obesity in the United States (Flegal et al., 2012; Schiller et 
al., 2012), and will likely represent an increasing demographic to undergo WLS (PickettBlakely et al., 2012). A systematic review of the literature revealed that besides the present study, only one other study (Conason et al.; 2012), attended to minority populations (Table 9). Furthermore, almost $40 \%$ of the studies reviewed in the systematic review did not include race/ethnicity information (Buffington, 2007; Dutta et al., 2006; Mauri et al., 2008; Mitchell et al., 2001; Suzuki et al., 2012; Tedesco et al., 2013; Wendling \& Wudyka, 2011) Thus, there is considerable need for additional research on WLS among ethnic/racial minority populations.

Future research considerations also warrant qualitative investigation of WLS patients who experience new-onset AUDs after WLS, as well as patients who experience an alleviation of problematic alcohol use after WLS. By gaining an in-depth understanding of factors that contributed to new onset AUDs (which is only possible with qualitative research methodologies) as well as factors that contributed to the alleviation of AUDS, risk and protective factors for post-WLS problematic alcohol use could be better elucidated. In turn, strengths-based approaches to preventing problematic alcohol use could be employed.

Finally, examining the association between pre-operative emotional eating and post-operative problematic alcohol use could also yield important findings. Emotional eating has been found to be prominent among overweight and obese individuals (Ricca et al., 2009), as well as bariatric surgery candidates (Fischer et al., 2007; Chesler et al., 2012). Furthermore, the qualitative data reported here revealed participants who engaged 
in emotional eating prior to WLS; however, after WLS they reported consuming alcohol instead of food in response to emotional distress.

\section{Conclusion}

This is the first study to examine young adult WLS patients' alcohol use and the factors surrounding an increase in alcohol use. By exploring factors that surround a WLS patient's increase in alcohol use, we gain an in-depth understanding of etiology, phenomenology, and motivations surrounding an increase in alcohol use after WLS. The findings reported here present important practice and policy implications to be considered, especially considering the emerging research that is documenting postoperative alcohol use problems among WLS patients.

\section{Limitations}

One limitation was a small sample size, particularly in regard to the quantitative portion of the study. The qualitative sample involved 12 participants, which has been determined to be a sufficient number of interviews to provide data saturation in social research (Guest, Bunce, \& Johnson, 2006). In fact, the researcher determined data saturation to be reached after 10 participants, but over-sampled due to the availability of interested participants.

A second limitation is that the generalizability of this study's findings is potentially limited as this is a convenience sample and the same bariatric surgeon treated all participants. However, because all patients were treated in the same facility with the same policies and messages regarding alcohol use, this is also strength of the study as it could potentially limit confounding variables. Selection bias remains a potential threat, as there is a chance that participants who did not want to participate in the study 
(quantitative or qualitative portions), are the ones who could be dealing with the most challenges post-surgery.

The study's reliance on retrospective, self-report data also poses a limitation, as participants might have either exaggerated or under-reported their responses for reasons including social desirability. However, self-report data are essential to health research (Stone et al., 2000). In addition, asking participants if their alcohol use "increased" "decreased" or "remained the same" is entirely subjective, and just because someone indicates an increase in alcohol use after WLS does not necessarily imply problematic drinking. Future research should obtain more detailed information regarding changes in alcohol use quantity and frequency after WLS. Of course, obtaining prospective alcohol use information (before and at multiple points after surgery) is even more ideal.

Efforts were made to address the self-report limitations of the study. First, by invoking in-depth, semi-structured interviews, a greater understanding was developed concerning an increase in alcohol use after WLS. Furthermore, the researcher has extensive research interviewing experience and is well versed at establishing rapport with research participants. The researcher also reminded participants that she was not affiliated with the bariatric surgery facility nor would the bariatric surgeon or staff read their confidential responses. She also reiterated to the participants that the project aims were to learn from the experiences, both good and bad, in order to help illicit truthful answers.

\section{Significance to Social Work}

Even considering the limitations of the study, the research presents important implications to the field of social work. With the increase in bariatric surgeries performed 
in the United States, coupled with the 2006 Center for Medicare and Medicaid services statement that announced coverage for both Medicare and Medicaid beneficiaries, social workers will have the opportunity to interact with weight loss surgery patients in a variety of settings such as hospitals, substance use facilities, and private practices. Furthermore, while current substance abuse or dependence is among the exclusion criteria for undergoing bariatric surgery (Mechanick et al., 2009), research indicates that as many as $80 \%$ of patients demonstrating problematic drinking behaviors still undergo bariatric surgery (Kudsi et al., 2013). In addition, as reported earlier, patients with a history of substance abuse might be especially at risk for relapse post-surgery (King et al., 2012; Suzuki et al., 2012). Thus, social workers will be uniquely positioned to help clients make decisions about treatment or changes in their lifestyle. Therefore, it is crucial for Social Workers to be well informed about potential addiction vulnerabilities post WLS. Knowledge of post-WLS research will also help social workers maintain a valuable role in interdisciplinary collaborations. 


\section{LIST OF REFERENCES}

Adams, C. E., Gabriele, J. M., Baillie, L. E., Dubbert, P. M. (2012). Tobacco use and substance use disorders as predictors of postoperative weight loss 2 years after bariatric surgery. The Journal of Behavioral Health Services \& Research, 39(4), 462-71. doi:http://dx.doi.org/10.1007/s11414-012-9277-z.

Abbatini F., Capoccia D., Casella G., Soricelli E., Leonetti F., Basso, N. (2013) LongTerm Remission Of Type 2 Diabetes In Morbidly Obese Patients After Sleeve Gastrectomy. Surgery For Obesity And Related Diseases, 9, 498-502.

Addolorato, G., Capristo, E., Greco, A. V., Stefanini, G. F., \& Gasbarrini, G. (1998). Influence of chronic alcohol abuse on body weight and energy metabolism: is excess ethanol consumption a risk factor for obesity or malnutrition?.Journal of Internal Medicine,244, 387-395.

Allen JP, Litten RZ, Fertig JB, Babor T (1997) A Review Of Research On The Alcohol Use Disorders Identification Test (Audit). Alcoholism: Clinical And Experimental Research, 21, 613-619.

Ambwani S, Boeka AG, Brown JD, Byrne TK, Budak AR, Sarwer DB, Fabricatore AN, Morey LC, O’Neil PM (2013). Socially Desirable Responding By Bariatric Surgery Candidates During Psychological Assessment. Surgery for Obesity and Related Diseases, 9(2),300-5.

American College Health Association. (2009). American College Health AssociationNational College Health Assessment Spring 2008 Reference Group Data Report (abridged): the American College Health Association. Journal Of American College Health, 57(5), 477.

Antin, T. M. J., \& Paschall, M. J. (2011). Weight perception, weight change intentions, and alcohol use among young adults. Body Image, 8, 149-156.

Arif, A. A., \& Roher, J. E. (2005). Patterns of alcohol drinking and its association with obesity: Data from the third national health and nutrition examination survey, 1988-1994. BMC Public Health, 5,126.

Armstrong, D., Gosling, A., Weinman, J., \& Marteau, T. (1997). The place of inter-rater reliability in qualitative research: an empirical study. Sociology, 31(3), 597-606.

Arnett, J. J. (2003). Conceptions of the transition to adulthood among emerging adults in American ethnic groups. New Directions For Child And Adolescent Development, 2003(100), 63-76. 
Arnett, J. J. (2000). Emerging adulthood: A theory of development from the late teens through the twenties. American Psychologist, 55(5), 469-480. doi:10.1037/0003066X.55.5.469

Auerbach, C.E., \& Silverstein, L.B. (2003). Qualitative data: An introduction to coding and analysis. New York: New York University Press.

Badaoui, A., De Saeger, C., Duchemin, J., Gihousse, D., De Timary, P., \& Stärkel, P. (2008). Alcohol dependence is associated with reduced plasma and fundic ghrelin levels. European Journal of Clinical Investigation, 38(6), 397-403. doi:10.1111/j.1365-2362.2008.01947.x

Bandealy, A., \& Stahl, C. (2012). Obesity, reproductive health, and bariatric surgery in adolescents and young adults. Journal of Pediatric and Adolescent Gynecology, 25(4), 277.

Barbour, R. S. (2001). Checklists for improving rigour in qualitative research: a case of the tail wagging the dog?. British Medical Journal, 322(7294), 1115-1117.

Bell, D. (2008). Destination drinking: Toward a research agenda on alcotourism. Drugs: education, prevention and policy, 15(3), 291-304.

Barry, D., \& Petry, N. M. (2009). Associations between body mass index and substance use disorders differ by gender: Results from the national epidemiologic survey on alcohol and related conditions. Addictive Behaviors, 34(1), 51-60. doi:10.1016/j.addbeh.2008.08.008

Barry, A. E., Piazza-Gardner, A. K., \& Holton, M. K. (2014). Assessing the alcohol-BMI relationship in a US national sample of college students. Health Education Journal, doi: 0017896914547289.

Bendsen, N. T., Christensen, R., Bartels, E. M., Kok, F. J., Sierksma, A., Raben, A., \& Astrup, A. (2012). Is beer consumption related to measures of abdonmial and general obesity? A systematic review and meta-analysis. Nutrition Reviews, 70(2), 67-87.

Blanco, C., Okuda, M., Wright, C., Hasin, D. S., Grant, B. F., Liu, S., \& Olfson, M. (2008). Mental health of college students and their non-college-attending peers: Results from the national epidemiologic study on alcohol and related conditions. Archives of General Psychiatry, 65(12), 1429-1437. 
Bray, G. A., Bouchard, C., \& James, W. P. T. (1998). Definition and proposed current classification of obesity. In G. A. Bray, C. Bouchard \& W. P. T. James (Eds.), Handbook of obesity. New York: Marcel Dekker.

Breslow, R. A., Chen, C. M., Graubard, B. I., Jacobovits, T., \& Kant, A. K. (2013). Diets of drinkers on drinking and nondrinking days: NHANES 2003-2008. The American Journal of Clinical Nutrition, 97, 1068-1075.

Breslow, R. A., \& Smothers, B. A. (2005). Drinking patterns and body mass index in never smokers: National health interview survey, 1997-2001. American Journal of Epidemiology, 161, 368-376.

Brown, S. A., McGue, M., Maggs, J., Schulenberg, J., Hingson, R., Swartzwelder, S., . . . Murphy, S. (2008). A developmental perspective on alcohol and youths 16 to 20 years of age. Pediatrics, 121, S290-S310. doi:10.1542/peds.2007-2243D

Buchwald, H., Avidor, Y., Braunwald, E., Jensen, M. D., Pories, W., Fahrbach, K., \& Schoelles, K. (2004). Bariatric surgery: A systematic review and meta-analysis. JAMA, the Journal of the American Medical Association, 292(14), 1724.

Buchwald, H. (2010). Metabolic surgery: A brief history and perspective. Surgery for Obesity and Related Diseases, 6(2), 221.

Buffington C (2007) Alcohol Use And Health Risks: Survey Results. Bariatric Times, $4: 21$.

Burkhauser, R. V., \& Cawley, J. (2008). Beyond BMI: The value of more accurate measures of fatness and obesity in social science research. Journal of Health Economics, 27(2), 519-529. doi:http://dx.doi.org/10.1016/j.jhealeco.2007.05.005

Calissendorff, J., Danielsson, O., Brismar, K., \& Röjdmark, S. (2005). Inhibitory effect of alcohol on ghrelin secretion in normal man. European Journal of Endocrinology, 152(5), 743-747. doi:10.1530/eje.1.01905

Centers for Disease Control and Prevention, U.S. Department of Health and Human Services. (2013, January 11). Vital signs: Bing drinking among women and high school girls-united states, 2011. Morbidity and Mortality Weekly Report, 62, 1.

Chen, K., \& Kandel, D. (1995). The natural history of drug use from adolescence to the mid-thirties in a general population sample. American Journal of Public Health, 85(1), 41. 
Charmaz, K. (2006). Constructing grounded theory: A practical guide through qualitative research. London: Sage Publications Ltd.

Chithambo, T. P., \& Huey, S. J. (2013). Black/white differences in perceived wieght and attractiveness among overweight women. Journal of Obesity, 2013 doi:10.1155/2013/320326

Chitwood DD, Sanchez J, Comerford M, Mccoy CB (2001) Primary Preventative Health Care Among Injection Drug Users, Other Sustained Drug Users, And Non-Users. Substance Use \& Misuse, 36, 807-824.

Conason, A., Teixeira, J., Hsu, C. H., Puma, L., Knafo, D., \& Geliebter, A. (2013). Substance use following bariatric weight loss surgery. JAMA surgery,148(2).

Contreras JE, Santander C, Court I, Bravo J (2013) Correlation Between Age And Weight Loss After Bariatric Surgery. Obesity Surgery, 23,1286.

Coulantuoni, C., Schweneker, J., McCarthy, P., Rada, P., Ladenheim, B., \& Cadet, J. (2001). Excessive sugar intake alters bidning to dopamine and mu-opioid receptors in the brain. Neuroreport, 12(3549), 3552.

Creswell, J. W. (2013). Research design: Qualitative, quantitative, and mixed methods approaches. Sage publications.

Creswell, J. W., Plano Clark, V. L., Gutmann, M. L., \& Hanson, W. E. (2003). Advanced mixed methods research designs. In A.Tashakkori \& C.Teddlie (Eds.), Handbook of mixed methods in social and behavioral research (pp. 209-240). Thousand Oaks, CA: Sage.

Creswell, J. W., \& Miller, D. L. (2000). Determining validity in qualitative inquiry.Theory into practice, 39(3), 124-130.

Cummings, D. E., Frayo, R. S., Marmonier, C., Aubert, R., \& Chapelot, D. (2004). Plasma ghrelin levels and hunger scores in humans initiating meals voluntarily without time- and food-related cues. American Journal of PhysiologyEndocrinology and Metabolism, 287, E297-E304. doi:10.1152/ajpendo.00582.2003

Dallongeville, J., Marécaux, N., Ducimetière, P., Ferrières, J., Arveiler, D., Bingham, A., Ruidavets, J.B., Simon, C., Amouyel, P. (1998). Influence of alcohol consumption and various beverages on waist girth and waist-to-hip ratio in a sample of french men and women. International Journal of Obesity Related Metabolism Disorders, 22(12), 1178-1183. 
Dawson DA, Grant BF, Stinson FS, Zhou Y (2005) Effectiveness of the Derived Alcohol Use Disorders Identification Test (AUDIT-C) in Screening for Alcohol Use Disorders and Risk Drinking in the US General Population. Alcoholism: Clinical and Experimental Research, 29 (5): 844-854.

Davenport, R. J., \& Wright, S. (2013). Treating obesity: Is it all in the gut? Drug Discovery Today, 12.

Davis, G. (2009). In Tucker L. (Ed.), The expert's guide to weight-loss surgery. New York, NY: The Penguin Group.

Davis, C., Curtis, C., Levitan, R. D., Carter, J. C., Kaplan, A. S., \& Kennedy, J. L. (2011). Evidence that 'food addiction' is a valid phenotype of obesity. Appetite, 57(3), 711-717. doi:http://dx.doi.org.ezproxy.fiu.edu/10.1016/j.appet.2011.08.017

Davis J.F., Schurdak J.D., Magriss I.J., Mul J.D., Grayson B.E., Pfluger P.T., Tschöp M.H., Seeley R.J., Benoit S.C. (2012) Gastric Bypass Surgery Attenuates Ethanol Consumption In Ethanol-Preferring Rats. Biological Psychiatry, 72,354-360.

De La Cruz-Munoz, N., Lopez-Mitnik, G., Arheart, K. L., Miller, T. L., Lipshultz, S. E., \& Messiah, S. E. (2013). Effectiveness of bariatric surgery in reducing weight and body mass index among hispanic adolescents. Obesity Surgery, 23(2), 150-156. doi:10.1007/s11695-012-0730-0

De la Cruz-Muñoz, N., Messiah, S. E., Cabrera, J. C., Torres, C., Cuesta, M., LopezMitnik, G., \& Arheart, K. L. (2010). Four-year weight outcomes of laparoscopic gastric bypass surgery and adjustable gastric banding among multiethnic adolescents. Surgery for Obesity and Related Diseases, 6(5), 542-547. doi:10.1016/j.soard.2010.06.004

Downe- Wamboldt, B. (1992). Content analysis: method, applications, and issues. Health Care For Women International, 13(3), 313-321.

Dorsey, R. R., Eberhardt, M. S., \& Ogden, C. L. (2010). Racial and ethnic differences in weight management behavior by weight perception status. Ethnicity \& Disease, 20(3), 244-250.

Duncan, A. E., Grant, J. D., Bucholtz, K. K., Madden, P. A., \& Heath, A. C. (2009). Relationship between body mass index, alcohol use, and alcohol misuse in a young adult female twin sample. Journal of Studies on Alcohol and Drugs, 70(445), 466. 
Duncan, G. J., Wilkerson, B., \& England, P. (2006). Cleaning up their act: the effects of marriage and cohabitation on licit and illicit drug use. Demography,43(4), 691710.

Egger, G., \& Boyd, S. (1997). An ecological approach to the obesity epidemic. British Medical Journal, 315, 477.

Ertelt, TW. Mitchell JE, Lancaster K, Crosby RD, Steffen KJ, Marino JM (2008) Alcohol Abuse And Dependence Before And After Bariatric Surgery: A Review Of The Literature And Report Of A New Data Set. Surgery for Obesity And Related Disease, 4(5): 647-650.

Ezzy, D. (2002). Qualitative analysis: Practice and innovation. London: Routledge.

Erikson, E. H. (1968). Identity: Youth and crisis. New York: Norton.

Fabricatore, A. N., Crerand, C. E., Wadden, T. A., Sarwer, D. B., \& Krasucki, J. L. (2006). How do mental health professionals evaluate candidates for bariatric surgery? Survery results. Obesity Surgery, 16, 567.

Finkelstein, E., Khavjou, O., Thompson, H., Trogdon, J., Pan, L., Sherry, B., \& Dietz, W. (2012). Obesity and severe obesity forecasts through 2030. American Journal of Preventive Medicine, 42(6), 563. doi:10.1016/S0749-3797(12)00266-8

Flegal, K. M., Carroll, M. D., Kit, B. K., \& Ogden, C. L. (2012). Prevalence of obesity and trends in the distribution of body mass index among US adults, 1999-2010 (report). JAMA, the Journal of the American Medical Association, 307(5), 491.

Flynn, K. J., \& Fitzgibbon, M. (1998). Body images and obesity risk among black females: A review of the literature. Annals of Behavioral Medicine, 20(1), 13-24.

Fogger, SA (2012) The Relationship Between Addictions And Bariatric Surgery For Nurses In Recovery. Perspectives In Psychiatric Care 48,10-15.

Fortuna, J. (2012). The obesity epidemic and food addiction: Clinical similarities to drug dependence. Journal of Psychoactive Drugs, 44(1), 56.

Gearhardt, A. N., \& Corbin, W. R. (2009). Body mass index and alcohol consumption: Family history of alcoholism as a moderator. Psychology of Addictive Behaviors, 23(2), 216-225. doi:10.1037/a0015011

Gearhardt, A. N., Corbin, W. R., \& Brownell, K. D. (2009). Preliminary validation of the Yale Food Addiction Scale. Appetite, 52(2), 430-436. 
Gold, M. S., Frost-Pineda, K., \& Jacobs, W. S. (2003). Overeating, binge eating, and eating disorders as addictions. Psychiatric Annals, 33(2), 117-122.

Gomez-Meade, C., Lopez-Mitnik, G., Messiah, S., Carrilo, A., \& de la Cruz-Munoz, N. (2013). Cardiometabolic health among gastric bypass surgery patients with polycystic ovarian syndrome. World J Diabetes, 4(3), 64.

Grabe, S., \& Hyde, J. S. (2006). Ethnicity and body dissatisfaction among women in the United States: A meta-analysis. Psychological Bulletin, 132(4), 622-640. doi:10.1037/0033-2909.132.4.622

Grant, B. F., Dawson, D. A., Sinson, F. S., Chou, S. P., Dufour, M. C., \& Pickering, R. P. (2004). The 12-month prevalence and trends in DSM-IV alcohol abuse and dependence: United States, 1991-1992 and 2001-2002. Drug and Alcohol Dependence, 74, 223-234.

Greenfield, T. K. (2001). Health disparities in alcohol-related disorders, problems, and treatment use by minorities. Front Lines: Linking Alcohol Services Research and Practice, 3(7).

Grucza, R. A., Krueger, R. F., Racette, S. B., Norberg, K. E., Hipp, P. R., \& Bierut, L. J. (2010). The emerging link between alcoholism risk and obesity in the United States. Archives of General Psychiatry, 67(12), 1301-1308.

Guest, G., Bunce, A., \& Johnson, L. (2006). How many interviews are enough? An experiment with data saturation and variability. Field Methods, 18(1), 59-82.

Guh, D., Zhang, W., Bansback, N., Amarsi, Z., Birmingham, C. L., \& Anis, A. (2009). The incidence of co-morbidities related to obesity and overweight: A systematic review and meta-analysis. BMC Public Health, 9(1), 88.

Hagerdorn, J. C., Encarnacion, B., Brat, G. A., \& Morton, J. M. (2007). Does gastric bypass alter alcohol metabolism? Surgery for Obesity and Related Diseases, 3, 543- 548.

Hajnal, A., Zharikov, A., Polston, J. E., Fields, M. R., Tomasko, J., Rogers, A. M., ... \& Thanos, P. K. (2012). Alcohol reward is increased after Roux-en-Y gastric bypass in dietary obese rats with differential effects following ghrelin antagonism.

Havin, G., DeLegge, M., \& Garrow, D. A. (2008). The impact of race on weight loss after roux-en-Y gastric bypass surgery. Obesity Surgery, 18, 39. 
Heinberg, L. J., \& Ashton, K. (2010). History of substance abuse relates to improved postbariatric body mass index outcomes. Surgery for Obesity and Related Diseases, 6(4), 417-421. doi:http://dx.doi.org/10.1016/j.soard.2010.04.001

Hendley, Y., Zhao, L., Coverson, D. L., Din-Dzietham, R., Morris, A., Quyyumi, A. A., . .. Vaccarino, V. (2011). Differences in weight perception among blacks and whites. Journal of Women's Health, 20(12), 1805-1811.

Heneghan, H. M., Cetin, D., Navaneethan, S. D., Orzech, N., Brethauer, S. A., \& Schauer, P. R. (2013). Effects of bariatric surgery on diabetic nephropathy after 5 years of follow-up. Surgery for Obesity and Related Diseases, 9(1), 7-14.

Hofmann, B. (2015). Obesity as a Socially Defined Disease: Philosophical Considerations and Implications for Policy and Care. Health Care Analysis, 1-15.

Hurt, R. T., Varayil, J. E., Mundi, M. S., Martindale, R. G., \& Ebbert, J. O. (2014). Designation of obesity as a disease: Lessons learned from alcohol and tobacco. Current Gastroenterology Reports, 16(11), 1-7.

Hsieh, H. F., \& Shannon, S. E. (2005). Three approaches to qualitative content analysis. Qualitative Health Research, 15(9), 1277-1288.

Jaunoo, S. S., \& Southall, P. J. (2009). Bariatric surgery. International Journal of Surgery, 8(2), 86-89.

Kenny, P. J. (2011). Common cellular and molecular mechanisms in obesity and drug addiction. Nature Reviews Neuroscience, 12(11), 638-651.

Kessler, R. C., Chiu, W. T., Demler, O., \& Walters, E. E. (2005). Prevalence, severity, and comorbidity of 12-month DSM-IV disorders in the National Comorbidity Survey Replication. Archives Of General Psychiatry, 62(6), 617-627

King, W. C., Chen, J., Mitchell, J. E., Kalarchian, M. A., Steffen, K. J., Engel, S. G., . . . Yanovski, S. Z. (2012). Prevalence of alcohol use disorders before and after bariatric surgery. JAMA: Journal of the American Medical Association, 307(23), 2516-2525.

Kleiner, K. D., Gold, M. S., Frost-Pineda, K., Lenz-Brunsman, B., Perri, M. G., \& Jacobs, W. S. (2004). Body mass index and alcohol use. Journal of Addictive Diseases, 23(3), 105-118.

Klockhoff H, Näslund I, Jones AW (2002) Faster Absorption Of Ethanol And Higher Peak Concentration In Women After Gastric Bypass Surgery. British Journal Of Clinical Pharmacology, 54(6): 587-591. 
Knight, J. R., Weschsler, H., Kuo, M., Seibring, M., Weitzman, E. R., \& Schuckit, M. (2002). Alcohol abuse and dependence among U.S. college students. Journal of Studies on Alcohol, 63, 263-270.

Koob, G. F., \& Le Moal, M. (2008). Addiction and the brain antireward system. Annual Review of Psychology, 59, 29-53.

Krauss, R. C., Powell, L. M., \& Wada, R. (2012). Weight misperceptions and racial and ethnic disparities in adolescent female body mass index. Journal of Obesity, 2012.

Lahti-Koski, M., Pietinen, P., Heliovaara, M., \& Vartiainen, E. (2002). Associations of body mass index and obesity with physical activity, food choices, alcohol intake, and smoking in the 1982-1997 FINRISK studies. The American Journal of Clinical Nutrition, 75, 809.

Leggio, L., Schwandt, M. L., Oot, E. N., Dias, A. A., \& Ramchandani, V. A. (2013). Fasting-induced increase in plasma ghrelin is blunted by intravenous alcohol administration: A within-subject placebo-controlled study. Psychoneuroendocrinology, 38(12), 3085-3091.

Lemon, S. C., Rosal, M. C., Zapka, J., Borg, A., \& Andersen, V. (2009). Contributions of weight perceptions to weight loss attempts: Differences by body mass index and gender. Body Image, 6(2), 90-96.

Lent, M. R., Hayes, S. M., Wood, G. C., Napolitano, M. A., Argyropoulos, G., Gerhard, G. S., ... \& Still, C. D. (2013). Smoking and alcohol use in gastric bypass patients. Eating behaviors, 14(4), 460-463.

Levinson, D. J. (1978). The seasons of a man's life. New York: Knopf.

Levinson, D. J. (1986). A conception of adult development. American Psychologist, 41(1), 3-13.

Levitsky, L. L., Misra, M., \& Boepple, P. A. (2009). Adolescent obesity and bariatric surgery. Current Opinions in Endocrinology, Diabetes, and Obesity, 16, 37.

Livingstone, A. (2011). Bariatric surgery significantly decreases the prevalence of type 2

diabetes mellitus and pre-diabetes among morbidly obese multiethnic adults: Long-term results. Journal of the American College of Surgeons, 212(4), 505-511. doi:10.1016/j.jamcollsurg.2010.12.015 
Lycett, D., Munafo, M., Johnstone, E., Murphy, M., \& Aveyard, P. (2011). Weight change over eight years in relation to alcohol consumption in a cohort of continuing smokers and quitters. Nicotine \& Tobacco Research, 13(11).

Mathers, C., Fat, D. M., \& Boerma, J. T. (2008). The global burden of disease: 2004 update. World Health Organization.

Mechanick JI, Kushner RF, Sugerman HJ, Gonzalez-Campoy JM, Collazo-Clavell ML, Spitz AF, Apovian CM, Livingston EH, Brolin R, Sarwer DB, Anderson WA, Dixon J (2009) American Association Of Clinical Endocrinologists, The Obesity Society, And American Society For Metabolic \& Bariatric Surgery Medical Guidelines For Clinical Practice For The Perioperative Nutritional, Metabolic, And Nonsurgical Support Of The Bariatric Surgery Patient. Obesity, 17(S1): S3-S72.

Messiah, S. E., Lopez-Mitnik, G., Winegar, D., Sherif, B., Arheart, K. L., Reichard, K. W., ... \& de la cruz-Muñoz, N. (2013). Changes in weight and co-morbidities among adolescents undergoing bariatric surgery: 1-year results from the Bariatric Outcomes Longitudinal Database. Surgery for Obesity and Related Diseases, 9(4), 503-513.

Mitchell JE, Lancaster KL, Burgard MA, Howell M, Krahn DD, Crosby RD, Wonderlich SA, Gosnell BA (2001) Long-Term Follow-Up Of Patients' Status After Gastric Bypass. Obesity Surgery, 11(4): 464.

Mitchell, J. E., Steffen, K. J., de Zwaan, M., Ertelt, T. W., Marino, J. M., \& Mueller, A. (2010). Congruence between clinical and research-based psychiatric assessment in bariatric surgical candidates. Surgery for Obesity and Related Diseases, 6(6), 628-634.

Moher, D., Liberati, A., Tetzlaff, J., \& Altman, D. G. (2009). Preferred reporting items for systematic reviews and meta-analyses: the PRISMA statement. Annals of Internal Medicine, 151(4), 264-269.

Mulia, N., Ye, Y., Greenfield, T. K., \& Zemore, S. E. (2009). Disparities in alcoholrelated problems among white, black, and hispanic americans. Alcoholism: Clinical and Experimental Research, 33(4), 654.

Nguyen NT, Masoomi H, Magno CP, Nguyen XT, Laugenour K, Lane J (2011) Trends In Use Of Bariatric Surgery, 2003-2008. Journal of The American College Of Surgeons, 213(2): 261-266. 
NIH consensus statement: Gastrointestinal surgery for severe obesity. (1991). Nutrition the International Journal of Applied and Basic Nutritional Sciences, 12(6), 397401.

Odom, J., Zalesin, K. C., Washington, T. L., Miller, W. W., Hakmeh, B., Zaremba, D. L., ... McCullough, P. A. (2010). Behavioral predictors of weight regain after bariatric surgery. Obesity Surgery, 20(3), 349-356. doi:http://dx.doi.org/10.1007/s11695-009-9895-6

Omalu BI, Ives DG, Buhar AM, Lindner JL, Schauer PR, Wecht CH, Kuller LH (2007) Death Rates And Causes Of Death After Bariatric Surgery For Pennsylvania Residents, 1995 To 2004. Archives Of Surgery, 142(10), Pp. 923.

Örnberg, J. C., \& Room, R. (2014). Impacts of tourism on drinking and alcohol policy in low-and middle-income countries: a selective thematic review.Contemporary Drug Problems, 41(2), 145-169.

Ostlund MP, Backman O, Marsk R, Stockeld D, Lagergren J, Rasmussen F, Näslund E (2013) Increased Admission For Alcohol Dependence After Gastric Bypass Surgery Compared With Restrictive Bariatric Surgery. JAMA Surgery, 148(4):374-377.

Padilla, M. B., Guilamo-Ramos, V., \& Godbole, R. (2012). A syndemic analysis of alcohol use and sexual risk behavior among tourism employees in Sosua, Dominican Republic. Qualitative health research, 22(1), 89-102.

Ouwens, M. A., Van Strein, T., \& van der Staak, C. F. (2003). Absence of a disinhibition effect of alcohol on food consumption. Eating Behaviors,114, 323-332.

Page, R. M., Scanlan, A., \& Allen, O. (1995). Adolescent perceptions of body weight and attractiveness: Important issues in alcohol and illicit drug use? Journal of Children and Adolescent Substance Abuse, 4, 43-55.

Pajari, M., Pietilainen, K. H., Kaprio, J., Rose, R. J., \& Saarni, S. E. (2010). The effect of alcohol consumption on later obesity in early adulthood-a population-based longitudinal study. Alcohol \& Alcoholism, 45, 173-179.

Palmqvist, R., \& Santavirta, N. (2006). What friends are for: The relationships between body image, substance use, and peer influence among finnish adolescents. Journal of Youth and Adolescence, 35, 203-217. 
Park, E. (2011). Overestimation and underestimation: Adolescents' weight perception in comparison to BMI-based weight status and how it varies across sociodemographic factors. Journal of School Health, 81(2), 57.

Parylak, S. L., Koob, G. F., \& Zorrilla, E. P. (2011). The dark side of food addiction. Physiology \& Behavior, 104(1), 149-156.

Patton, M. Q. (2002). Qualitative research and evaluation methods, 3rd edition. London: Sage.

Pepino, M. Y., \& Mennella, J. A. (2007). Effects of cigarette smoking and family history of alcoholism on sweet taste perception and food cravings in women. Alcoholism: Clinical and Experimental Research, 31(11), 1891-1899. doi:10.1111/j.15300277.2007.00519.x

Pepino, M. Y., Okunade, A. L., Eagon, J. C., Bartholow, B. D., Bucholz, K., \& Klein, S. (2015). Effect of Roux-en-Y Gastric Bypass Surgery: Converting 2 Alcoholic Drinks to 4. JAMA surgery. Published online August 05, 2015. doi:10.1001/jamasurg.2015.188

Pickett-Blakely, O. E., Huizinga, M. M., \& Clark, J. M. (2012). Sociodemographic trends in bariatric surgery utilization in the USA. Obesity Surgery, 22, 838.

Polivy J., \& Herman, C. P. (1976). The effects of alcohol on eating behavior: Disinhibition or sedation? Addictive Behaviors, 1(121), 125.

Powell, A. D., \& Kahn, A. S. (1995). Racial differences in women's desires to be thin. International Journal of Eating Disorders, 17(2), 191-195.

Power, C., \& Estaugh, V. (1990). The role of family formation and dissolution in shaping drinking behaviour in early adulthood. British Journal of Addiction, 85, 521-530

Rada, P., Avena, N. M., \& Hoebel, B. G. (2005). Daily bingeing on sugar repeatedly releases dopamine in the accumbens shell. Neuroscience, 134(737), 744.

Rahman, M., \& Berenson, A. B. (2010). Self-perception of weight and its association with weight-related behaviors in young reproductive-age women. Obstetrics \& Gynecology, 116(6), 1274-1280.

Reedy, S. (2009). An evidence-based review of obesity and bariatric surgery. The Journal for Nurse Practitioners, 5(1), 22-29. doi:10.1016/j.nurpra.2008.07.017 
Reslan S, Saules KK, Greenwald MK, Scuh LM (2014) Substance Misuse Following Roux-En-Y-Gastric Bypass Surgery. Substance Use And Misuse 49,405-417.

Rothman, K. J. (2008). BMI-related errors in the measurement of obesity. International Journal of Obesity, 32, S56-S59.

Rummel CM, Heinberg LJ(2014) Assessing Marijuana Use In Bariatric Surgery Candidates: Should It Be A Contradiction? Obesity Surgery 10,1764-70.

Saber, A. A., Elgamal, M., H., \& McLeod, M., K. (2008). Bariatric surgery: The past, present, and future. Obesity Surgery, 18, 121-128.

Saguy, A. C. (2013). What's wrong with fat?.New York: Oxford.

Saldano, J. (2009). The coding manual for qualitative researchers. London: Sage Publications.

Saules KK, Wiedemann A, Ivezah V, Hopper JA, Foster-Hartsfield J, Schwartz D (2010) Bariatric Surgery History among Substance Abuse Treatment Patients: Prevalence and Associated Features. Surgery for Obesity and Related Diseases 6, 615-621.

Schellekens, H., Dinan, T. G., \& Cryan, J. F. (2012). Ghrelin at the interface of obesity and reward. Vitamins And Hormones, 91, 285-323.

Schiller, J. S., Lucas, J. W., \& Peregoy, J. A. (2014). Summary health statistics for US adults: National Health Interview Survey, 2011. Hyattsville, MD: National Center for Health Statistics; 2012.

Schlegel, A., \& Barry, H., III. (1991). Adolescence: An Anthropological Inquiry. New York: Free Press.

Smith, R. R., Hong, J., Harvey, A. E., Lewis, T., Diaz, D., \& Nunez, N. P. (2008). Ethanol consumption does not promote weight gain in female mice. Annals of Nutrition \& Metabolism, 53, 252-259.

Smith, S. C. J. (2007). Multiple risk factors for cardiovascular disease and diabetes mellitus. American Journal of Medicine, 120, S3-S11.

Spadola, C. E., Wagner, E. F., Dillon, F. R., Trepka, M. J., La Cruz- Munoz, D., \& Systematic Review of the Emerging Research and Its Implications. Alcoholism: Clinical and Experimental Research, 39(9), 1582-1601. 
Steffen, K.J., Engel, S.G., Pollert, G.A., Li, C. And Mitchell, J.E., Blood Alcohol Concentrations Rise Rapidly And Dramatically After Roux-En-Y Gastric Bypass. Surgery For Obesity And Related Diseases 9:470-473.

Stice, E. (1994). Review of the evidence for a sociocultural model of bulimia nervosa and an exploration of the mechanisms of action. Clinical Psychology Review, 14 (7), 633-661.

Stone, A. L., Becker, L. G., Huber, A. M., \& Catalano, R. F. (2012). Review of risk and protective factors of substance use and problem use in emerging adulthood. Addictive Behaviors, 37(7), 747-775.

Strauss, A., \& Corbin, J. (1998). Basics of qualitative research: Procedures and techniques for developing grounded theory. ed: Thousand Oaks, CA: Sage.

Substance Abuse and Mental Health Services Administration, Results from the 2013 National Survey on Drug Use and Health: Summary of National Findings, NSDUH Series H-48, HHS Publication No. (SMA) 14-4863. Rockville, MD: Substance Abuse and Mental Health Services Administration, 2014.

Sudan, R., Winegar, D., Thomas, S., \& Morton, J. (2014). Influence of ethnicity on the efficacy and utilization of bariatric surgery in the USA. Journal of Gastrointestinal Surgery, 18(1), 130-136.

Suzuki, J., Haimovici, F., \& Chang, G. (2012). Alcohol use disorders after bariatric surgery. Obesity Surgery, 22(2), 201-207. doi:10.1007/s11695-010-0346-1

Svensson, P., Anveden, Å, Romeo, S., Peltonen, M., Ahlin, S., Burza, M.A., Carlsson, B., Jacobson, P., Lindroos, A., Lönroth, H., Maglio, C., Näslund, I., Sjöholm, K., Wedel, H., Söderpalm, B., Sjöström, L. And Carlsson, L.M.S.(2013) Alcohol Consumption And Alcohol Problems After Bariatric Surgery In The Swedish Obese Subjects Study. Obesity, 21(12): 2444-2451.

Tashakkori, A., \& Teddlie, C. (2003). Handbook On Mixed Methods In The Behavioral and Social Sciences. Thousand Oaks, CA: Sage.

Tedesco, M., Hua, W. Q., Lohnberg, J. A., Bellatorre, N., \& Eisenberg, D. (2013). A prior history of substance abuse in veterans undergoing bariatric surgery. Journal of Obesity, 2013, 1.

Thanos, P.K., Subrize, M., Delis, F., Cooney, R.N., Culnan, D., Sun, M., Wang, G., Volkow, N.D. And Hajnal, A. (2012) Gastric Bypass Increases Ethanol And 
Water Consumption In Diet-Induced Obese Rats. Obesity Surgery, 22(12), Pp. 1884-1892.

Thomson, C. A., Wertheim, B. C., Hingle, M., Wang, L., Neuhouser, M. L., Gong, Z., Manson, J. E. (2012). Alcohol consumption and body weight change in postmenopausal women: Results from the women's health initiative. International Journal of Obesity, 36, 1158-1164.

Tolstrup, J. S., Heitmann, B. L., Tjonneland, A. M., Overvad, O. K., Sorensen, T. A., \& Gronbaek, M. N. (2005). The relation between drinking pattern and body mass index and waist and hip circumference. International Journal of Obesity Related Metabolism Disorders, 29(5), 490-497.

Trayhurn, P., \& Beattie, J. H. (2001). Physiological role of adipose tissue: White adipose tissue as an endocrine and secretory organ. Proceedings of the Nutrition Society, 60(3), 3129.

Turner, P. L., Oyetunji, T. A., Gantt, G., Chang, D. C., Cornwell, E. E., \& Fullum, T. M. (2011). Demographically associated variations in outcomes after bariatric surgery. The American Journal of Surgery, 201(4), 475-480.

U.S. Department of Health and Human Services, National Institutes of Health, National Institute on Alcohol Abuse and Alcoholism (2006, April). Young adult drinking (Alcohol Alert No. 68). Retrieved from http://pubs.niaaa.nih.gov/publications/aa68/aa68.htm

Volkow, N. D., Wang, G. J., Fowler, J. S., \& Telang, F. (2008). Overlapping neuronal circuits in addiction and obesity: Evidence of systems pathology. Philosophical Transactions of the Royal Society, 363(150

Wallace, A. E., Young-Xu, Y., Hartley, D., \& Weeks, W. B. (2010). Racial, socioeconomic, and Rural-Urban disparities in obesity-related bariatric surgery. Obesity Surgery, 20, 1354.

Wang, G. J., Volkow, N. D., Telang, F., Jayne, M., Ma, J., Rao, M., ... \& Fowler, J. S. (2004). Exposure to appetitive food stimuli markedly activates the human brain. Neuroimage, 21(4), 1790-1797.

Wee, C.C., Mukamal, K.J., Huskey, K.W., Davis, R.B., Colten, M.E., Bolcic-Jankovic, D., Apovian, C.M., Jones, D.B. And Blackburn, G.L. (2014) High Risk Alcohol Use After Weight Loss Surgery. Surgery for Obesity and Related Diseases 10 (3): 508-13 
Weller, W., Rostani, C., \& Hannan, E. (2003). Predictors of in-hospital postoperative complications among adults undergoing bariatric procedures in New York State. Obesity Surgery, 16, 702.

Woodard, G. A., Downey, J., Hernandez-Boussard, T., \& Morton, J. M. (2011). Impaired alcohol metabolism after gastric bypass surgery: A case-crossover trial. Journal of the American College of Surgeons, 212(2), 209-214.

doi:10.1016/j.jamcollsurg.2010.09.020

Wren, A. M., Seal, L. J., Cohen, M. A., Brynes, A. E., Frost, G. S., Murphy, K. G., . . . Bloom, S. R. (2001). Ghrelin enhances appetite and increases food intake in humans. The Journal of Clinical Endocrinology \& Metabolism, 86(12), 5992.

Yeomans, M. R. (2010). Alcohol, appetite and energy balance: Is alcohol intake a risk factor for obesity. Physiology \& Behavior, 100, 82-89.

Yeomans, M. R., Hails, N. J., \& Nesic, J. S. (1999). Alcohol and the appetizer effect. Behavioral Pharmacology, 10, 151-161.

Yoon, Y. H., Yi, H. Y., Grant, B. F., \& Dufour, M. C. (2001). Surveillance Report\# 57: liver cirrhosis mortality in the United States, 1970-98. NIAAA, Division of Biometry and Epidemiology, Alcohol Epidemiologic Data System, Bethesda, MD. 
APPENDICES 
TABLE 2

Characteristics of Published Studies examining Alcohol and Illicit Drug Use in Post-Bariatric Populations (Tables listed in order of appearance in the manuscript)

\begin{tabular}{|lllll}
\hline Study & Research Design & Sample & Methods & Substance Use Outcomes \\
& a. Study Design & a. $N$ & a. Assessment instrument(s) & Measured \\
& b. Assessment Time & b. Recruitment Design & b. Assessment method(s) & \\
& Points & c. Inclusion Criteria & & \\
& & d. Baseline Response Rate & \\
& e. Retention Rate & \\
& f. Surgery Type & \\
& g. Age & \\
& h. Sex & \\
& i. Race/Ethnicity & \\
\end{tabular}




\begin{tabular}{|c|c|c|c|c|c|}
\hline $\begin{array}{l}\text { Svensson et al., } \\
2013\end{array}$ & 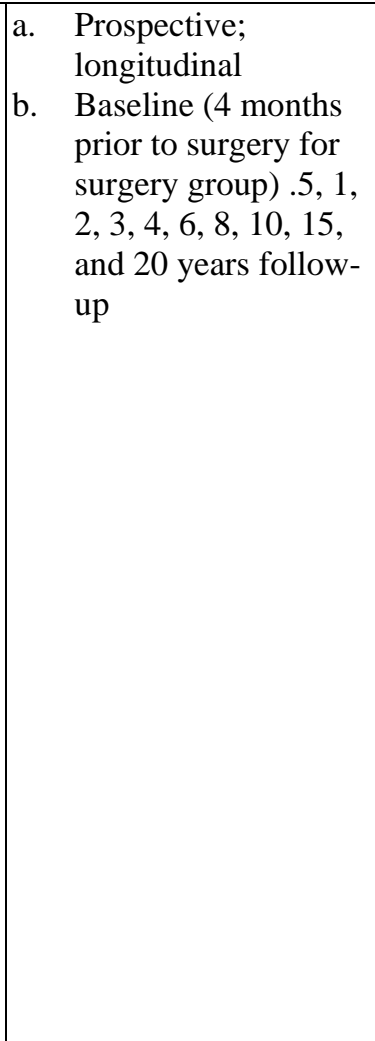 & & $\begin{array}{l}\text { Surgery group =2,010; } \\
\text { non-surgical control group = } \\
2,037 \\
\text { Sample derived from } \\
\text { Swedish Obese Subjects } \\
\text { (SOS) trial; recruited from } \\
\text { media campaigns and health } \\
\text { care centers } \\
\text { Identical inclusion/exclusion } \\
\text { criteria for both surgery and } \\
\text { control groups: eligible for } \\
\text { bariatric surgery, between } 37 \\
\text { and } 60 \text { years; BMI } \geq 34 \text { men } \\
\text { and } \geq 38 \text { for women } \\
\text { n.d. } \\
\text { n.d. .5 yrs., n.d. } 1 \text { yr., } \\
\text { 87\% } 2 \text { yrs., } 71 \% 10 \text { yrs., } 52 \% \\
\text { 15 yrs., 50\% } 20 \text { yrs. } \\
\text { GBP = 265; 376=AGB; } \\
\text { VBG =1,369 } \\
\text { Mean age (S.D.): } \\
\text { GBP= 47 (6.0); AGB=47.6 } \\
\text { (6.0); VBG=47.1 (5.9) } \\
\text { Males: GBP=28.7 \%, } \\
\text { AGB=30.9 \%, VBG=29.1 \% } \\
\text { N.D. (conducted in Sweden) }\end{array}$ & $\begin{array}{l}\text { a. SOS dietary questionnaire (assessed } \\
\text { intake of food and beverages in past } \\
3 \text { months); self-reported alcohol use } \\
\text { problems were assessed with an } \\
\text { affirmative response to "Do you } \\
\text { think you have alcohol problems?”; } \\
\text { alcohol abuse diagnosis obtained } \\
\text { from medical records } \\
\text { b. Self-reported data (alcohol use } \\
\text { frequency and problems with } \\
\text { alcohol) and chart review (alcohol } \\
\text { abuse diagnosis) N.D. provided if } \\
\text { assessments were self-administered } \\
\text { or interviewer/ } \\
\text { clinician administered }\end{array}$ & $\begin{array}{ll}\text { 1. } & \begin{array}{l}\text { Post-surgical } \\
\text { problematic } \\
\text { alcohol } \\
\text { use/alcohol } \\
\text { use disorder } \\
\text { 2. }\end{array} \begin{array}{l}\text { Post-surgical } \\
\text { alcohol use/ } \\
\text { frequency of } \\
\text { alcohol use } \\
\text { 3. Quantity of } \\
\text { alcohol } \\
\text { intake }\end{array}\end{array}$ \\
\hline
\end{tabular}




\begin{tabular}{|c|c|c|c|c|}
\hline King et al., 2012 & \begin{tabular}{|ll} 
a. & Prospective; \\
& longitudinal \\
b. & Baseline (pre- \\
& surgery), 1, and 2 \\
& years post-surgery
\end{tabular} & \begin{tabular}{|ll} 
a. & $\mathrm{n}=1945$ \\
b. & Sample recruited from \\
& participating surgeons at 10 \\
& centers across the U.S. \\
c. & Patients > 18 years old, \\
& seeking bariatric surgery \\
d. & $79 \%$ completed baseline and \\
& first and/or $2^{\text {nd }}$ year post- \\
& operative assessment \\
e. & See above \\
f. & $69.6 \%=$ RYGB, \\
& $25.2 \%=$ AGB \\
& $4.9 \%=$ other \\
g. & Median age (range)=47 (38- \\
& $55)$ \\
h. & $78.8 \%=$ female; $21.2 \%=m a l e$ \\
i. & $87 \%$ white; $9.4 \%$ black; $4.9 \%$ \\
& Hispanic
\end{tabular} & $\begin{array}{ll}\text { a. } & \text { Alcohol Use Disorders } \\
\text { Identification Test (AUDIT); } \\
\text { scored an } 8 \text { or higher on } \\
\text { AUDIT, or at least } 1 \text { symptom } \\
\text { of alcohol related harm or } \\
\text { alcohol dependence } \\
\text { b. }\end{array}$ & $\begin{aligned} \text { 1. } & \begin{array}{l}\text { Pre-surgical } \\
\text { history of } \\
\text { problematic } \\
\text { alcohol use/ } \\
\text { alcohol use }\end{array} \\
\text { disorder } & \\
\text { 2. } & \text { Post-surgical } \\
& \text { problematic } \\
& \text { alcohol } \\
& \text { use/alcohol use } \\
& \text { disorder } \\
\text { 3. } & \begin{array}{l}\text { Post-surgical } \\
\text { alcohol use/ } \\
\text { frequency of } \\
\text { alcohol use }\end{array} \\
\text { 4. } & \begin{array}{l}\text { Quantity of } \\
\text { alcohol intake }\end{array}\end{aligned}$ \\
\hline $\begin{array}{l}\text { Conason et al., } \\
2012\end{array}$ & \begin{tabular}{|ll} 
a. & Prospective; \\
& longitudinal \\
b. & Baseline (pre- \\
& surgery) and 1, 3, 6, \\
& 12, and 24 months \\
& post-surgery
\end{tabular} & $\begin{array}{ll}\text { a. } & \mathrm{n}=155 \\
\text { b. } & \text { Recruited from a pre- } \\
& \begin{array}{l}\text { operation information center } \\
\text { at one participating bariatric }\end{array} \\
& \text { surgery center } \\
\text { c. } & \text { N.D. } \\
\text { d. } & \text { N.D. } \\
\text { e. } & 61 \% 1 \text { mo., } 41 \% 3 \text { mo., } 43 \% \\
& 6 \text { mo., } 49 \% 12 \text { mo., } 25 \% 24 \\
& \text { mo. } \\
\text { f. } & n=100 \text { RYGB; n=55 AGB } \\
\text { g. } & \text { Mean age = 40 (SD=11) } \\
\text { h. } & 85 \% \text { female, } 15 \% \text { male } \\
\text { i. } & 47 \% \text { Hispanic, 32\% non- } \\
& \text { Hispanic black,17\% non- } \\
& \text { Hispanic white 1\% Asian; 3\% } \\
& \text { other }\end{array}$ & $\begin{array}{l}\text { a. "Compulsive Behaviors } \\
\text { Questionnaire” (developed by study } \\
\text { authors) } \\
\text { b. }\end{array}$ & 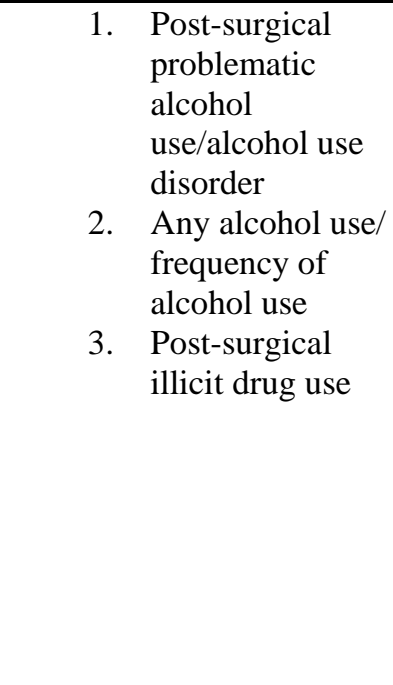 \\
\hline
\end{tabular}




\begin{tabular}{|c|c|c|c|c|c|}
\hline Wee et al., 2014 & 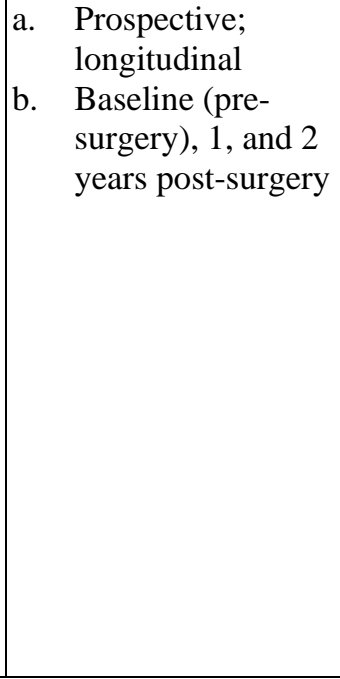 & $\begin{array}{ll}\text { a. } & \text { n= 541 } \\
\text { b. } & \text { Sample derived from } \\
& \text { Assessment of Bariatric } \\
& \text { Surgery Study (ABS); } \\
& \text { recruited from } 2 \text { WLS centers } \\
& \text { in Boston, MA. } \\
\text { c. } & \text { 18-65, English speaking, } \\
& \text { permission of physician } \\
\text { d. } & 70 \% \\
\text { e. } & 69 \% 1 \text { year, } 63 \% 2 \text { year } \\
\text { f. } & \text { RYBG = 55\%, AGB = 44\%; } \\
& \text { ASG/other = 1\% } \\
\text { g. } & \text { Mean age = 43.9 } \\
\text { h. } & \text { Male = 24 \%; Female = 76\% } \\
\text { i. } & 69 \% \text { non-Hispanic white; } \\
& \text { 17\% African American; } 11 \% \\
& \text { Hispanic; } 4 \% \text { Other }\end{array}$ & & $\begin{array}{l}\text { Alcohol Use Disorders } \\
\text { Identification Test - C } \\
\text { (AUDIT-C); high-risk alcohol } \\
\text { use defined as cut off score } \geq 4 \\
\text { in men } \geq 3 \text { in women, or } \\
\text { affirmative response to follow } \\
\text { up items of alcohol dependency } \\
\text { (i.e., impaired control over } \\
\text { drinking, morning drinking, } \\
\text { etc.), or harmful drinking (i.e., } \\
\text { others concerned about } \\
\text { drinking, guilt after drinking, } \\
\text { etc.) . } \\
\text { Self-reported; Telephone } \\
\text { interviews }\end{array}$ & $\begin{array}{l}\text { 1. } \begin{array}{l}\text { Post-surgical } \\
\text { problematic } \\
\text { alcohol } \\
\text { use/alcohol use } \\
\text { disorder }\end{array} \\
\text { 2. Any alcohol use/ } \\
\text { frequency of } \\
\text { alcohol use } \\
\text { 3. } \begin{array}{l}\text { Quantity of } \\
\text { alcohol intake }\end{array}\end{array}$ \\
\hline
\end{tabular}




\begin{tabular}{|c|c|c|c|c|}
\hline Lent et al., 2013 & \begin{tabular}{|ll} 
a. & $\begin{array}{l}\text { Prospective; } \\
\text { b. }\end{array}$ \\
longitudinal \\
Baseline (6-12 \\
months before \\
surgery) \& 1 time \\
post-surgery (mean \\
34.9 months post- \\
surgery; +/ 12.8 \\
months)
\end{tabular} & 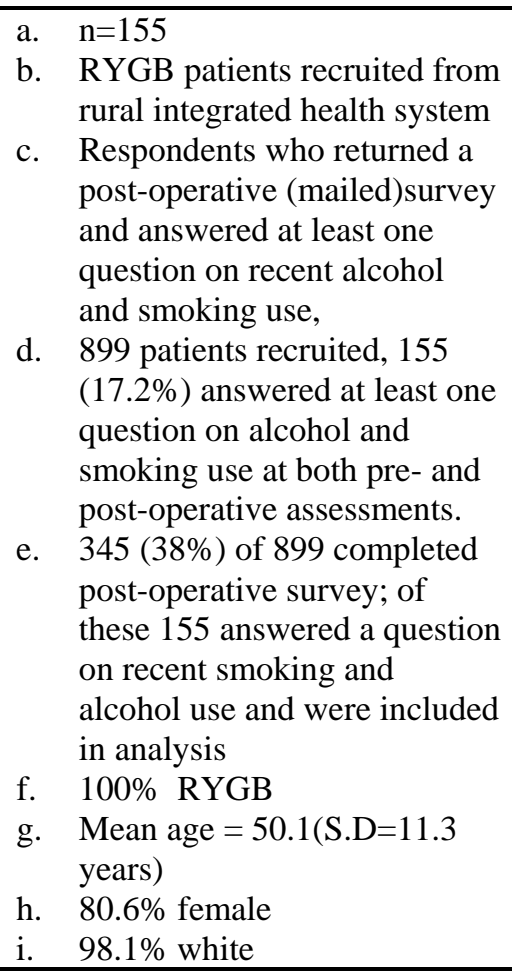 & $\begin{array}{l}\text { a. Substance use questionnaire } \\
\text { created by study researchers } \\
\text { (not validated). Questions } \\
\text { included: "How often did you } \\
\text { have a drink containing alcohol } \\
\text { in the past year?” "How many } \\
\text { drinks did you have on a typical } \\
\text { day when you were drinking in } \\
\text { the past year?” and "How often } \\
\text { did you have } 6 \text { or more drinks } \\
\text { on [one] occasion in the past } \\
\text { year?” Alcohol use on typical } \\
\text { drinking occasions categorized } \\
\text { as } 1 \text { ) none, 2) low consumption } \\
\text { (1-4 drinks), and 3) high } \\
\text { consumption ( } \geq 5 \text { ). } \\
\text { Mailed, self-administered } \\
\text { questionnaire }\end{array}$ & $\begin{array}{l}\text { 1. Any alcohol use/ } \\
\text { frequency of } \\
\text { alcohol use }\end{array}$ \\
\hline $\begin{array}{l}\text { Alfonsson et al., } \\
2014\end{array}$ & $\begin{array}{ll}\text { a. } & \text { Prospective; } \\
& \text { longitudinal } \\
\text { b. } & 5 \text { months prior } \\
& \text { to surgery ( } m- \\
& 153 \text { days, } \\
\text { SD=10.52) and } \\
12 \text { months post- } \\
\text { surgery ( } m=370, \\
\text { SD=77.3)post- } \\
\text { surgery }\end{array}$ & $\begin{array}{ll}\text { a. } & \text { n=129 } \\
\text { b. } & \text { Patients were recruited from } \\
& \text { a University hospital in } \\
& \text { Sweden who were eligible for } \\
& \text { RYGB } \\
\text { c. } & \text { N.D. } \\
\text { d. } & \text { N.D } \\
\text { e. } & 100 \% \text { (only complete data } \\
& \text { used in analyses). } \\
\text { f. } & 100 \% \text { RYGB } \\
\text { g. } & \text { Mean age =42.8 (SD=10.52) } \\
\text { h. } & 78 \% \text { female } \\
\text { i. } & \text { N.D. (conducted in Sweden) }\end{array}$ & $\begin{array}{ll}\text { a. } & \text { Alcohol Use Disorders } \\
& \text { Identification Test (AUDIT); } \\
\text { scored an } 8 \text { (men) or } 6 \text { (women) or } \\
\text { higher on AUDIT to indicate AUD } \\
\text { or at least } 1 \text { symptom of alcohol } \\
\text { related harm or alcohol } \\
\text { dependence; Adult ADHD Self- } \\
\text { Rating Scale (ASRS) for ADHD } \\
\text { symptomatology } \\
\text { b. } \\
\text { Self-reported data; N.D. if } \\
\text { assessments were self-administered } \\
\text { or interviewer/clinician } \\
\text { administered }\end{array}$ & $\begin{array}{ll}\text { 1. } & \text { History of } \\
& \text { problematic alcohol } \\
& \text { use/alcohol use } \\
& \text { disorder } \\
2 . & \text { Post-surgical } \\
& \text { problematic alcohol } \\
& \text { use/alcohol use } \\
& \text { disorder }\end{array}$ \\
\hline
\end{tabular}




\begin{tabular}{|c|c|c|c|c|}
\hline Buffington, 2007 & $\begin{array}{ll}\text { a. } & \text { Cross-sectional } \\
\text { b. } & \text { One time post- } \\
& \text { surgery; } 84 \% \geq \\
& \text { one year post- } \\
& \text { surgery }\end{array}$ & $\begin{array}{ll}\text { a. } & \mathrm{n}=318 \\
\text { b. } & \text { Online survey } \\
\text { c. } & \text { Open to all readers of an } \\
& \text { online bariatric surgery } \\
& \text { journal, who had bariatric } \\
& \text { surgery } \\
\text { d. } & \text { N/A } \\
\text { e. } & \text { N/A } \\
\text { f. } & 97.4 \% \text { RYGB } \\
\text { g. } & 49.4 \% \text { between } 36 \text { and } 50 \\
\text { h. } & 93.7 \% \text { female (n=298) } \\
\text { i. } & \text { N.D.; respondents from U.S., } \\
& \text { northern Europe, \& Israel }\end{array}$ & $\begin{array}{l}\text { a. } \\
\text { No standardized assessments; } \\
\text { "questions pertaining to alcohol } \\
\text { sensitivity, postoperative changes } \\
\text { in alcohol use and clearance." } \\
\text { b. Online survey }\end{array}$ & 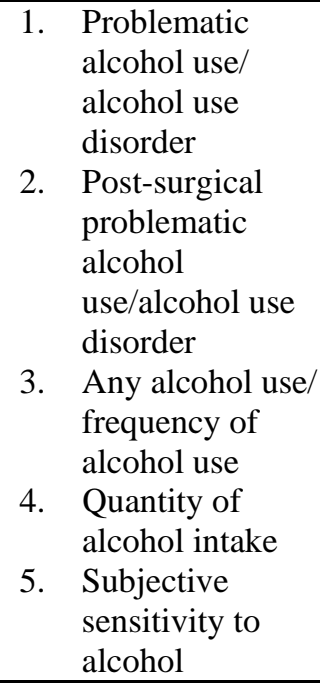 \\
\hline Ertelt et al., 2008 & $\begin{array}{|ll|}\text { a. } & \text { Cross-sectional } \\
\text { b. } & \text { One time; 6-10 years } \\
& \text { post-bariatric surgery }\end{array}$ & $\begin{array}{ll}\text { a. } & \mathrm{n}=70 ; \\
\text { b. } & \text { Surveys mailed to patients } \\
& \text { who underwent the RYGB } \\
& \text { procedure, 6-10 years ago, at } \\
& \text { one bariatric surgery center } \\
\text { c. } & \text { N.D. } \\
\text { d. } & 28 \% \text { ( } 70 \text { of } 250 \text { mailed } \\
& \text { surveys) } \\
\text { e. } & \text { N/A } \\
\text { f. } & 100 \%=\text { RYGB } \\
\text { g. } & \text { Mean age=49.9 (SD=9.2) } \\
\text { h. } & 85.5 \% \text { female } \\
\text { i. } & 90 \% \text { white }\end{array}$ & $\begin{array}{l}\text { a. Post-Bariatric Surgery Appearance } \\
\text { Questionnaire (developed by study } \\
\text { authors, contains questions on } \\
\text { alcohol abuse and dependence } \\
\text { based on DSM-IV criteria; not } \\
\text { validated) } \\
\text { b. Self-administered questionnaires } \\
\text { mailed to participants }\end{array}$ & $\begin{array}{l}\text { 1. } \begin{array}{l}\text { History of } \\
\text { problematic } \\
\text { alcohol use/ } \\
\text { alcohol use } \\
\text { disorder }\end{array} \\
\text { 2. } \begin{array}{l}\text { Post-surgical } \\
\text { problematic } \\
\text { alcohol } \\
\text { use/alcohol use } \\
\text { disorder }\end{array} \\
\text { 3. Any alcohol use/ } \\
\text { frequency of } \\
\text { alcohol use } \\
\text { 4. } \begin{array}{l}\text { Quantity of } \\
\text { alcohol intake }\end{array} \\
\text { 5. Subjective } \\
\text { sensitivity to } \\
\text { alcohol }\end{array}$ \\
\hline
\end{tabular}




\begin{tabular}{|c|c|c|c|c|}
\hline Mitchell et al., 2001 & $\begin{array}{ll}\text { a. } & \text { Cross-sectional } \\
\text { b. } & \text { One time; 13-15 } \\
& \text { years post- } \\
& \text { surgery }\end{array}$ & $\begin{array}{ll}\text { a. } & \mathrm{n}=78 \\
\text { b. } & \text { Post-operative patients from } \\
& \text { one facility, contacted via } \\
& \text { postal mail and telephone } \\
\text { c. } & \text { N.D. } \\
\text { d. } & 78 \% \text { (100 post-operative } \\
& \text { patients attempted to be } \\
& \text { contacted) } \\
\text { e. } & \text { N/A } \\
\text { f. } & 100 \% \text { RYGB } \\
\text { g. } & \text { Mean age = 56.8 (31-77) } \\
\text { h. } & 83 \%(n=65) \text { female } \\
\text { i. } & \text { N.D. }\end{array}$ & $\begin{array}{ll}\text { a. } & \text { MFED - instrument which uses } \\
\text { sections of Structured Clinical } \\
\text { Interview for DSM-IV } \\
\text { Disorders (SCID) } \\
\text { b. } \\
\end{array}$ & 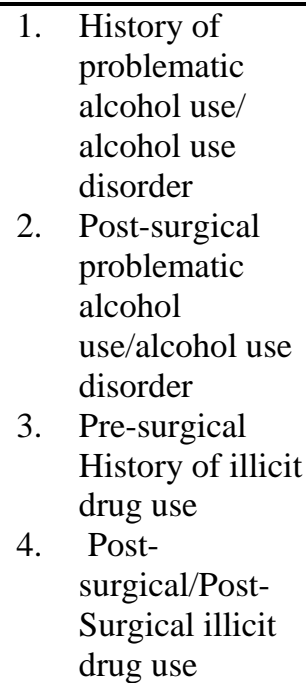 \\
\hline $\begin{array}{l}\text { Macias \& Leal, } \\
2003\end{array}$ & $\begin{array}{ll}\text { a. } & \text { Cross-sectional } \\
\text { b. } & \text { One time; } 18 \\
& \text { months post- } \\
& \text { surgery }\end{array}$ & $\begin{array}{ll}\text { a. } & \mathrm{n}=140 \\
\text { b. } & \text { Post-operative patients from a } \\
& \text { University hospital in Spain } \\
\text { c. } & \text { N.D. } \\
\text { d. } & \text { N.D. } \\
\text { e. } & \text { N/A } \\
\text { f. } & \text { 100\% VBG } \\
\text { g. } & \text { Binge eating group } 36.46 \\
& \text { (SD=11.72); non binge eating } \\
& \text { eating group 44.61 (SD=9.92) } \\
\text { h. } & 78.6 \% \text { female } \\
\text { i. } & \text { N.D. (conducted in Spain) }\end{array}$ & 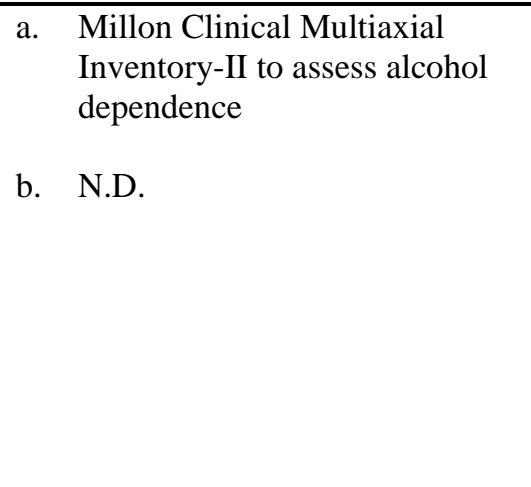 & $\begin{array}{ll}1 . & \text { Post-surgical } \\
\text { problematic } \\
\text { alcohol } \\
\text { use/alcohol use } \\
\text { disorder }\end{array}$ \\
\hline
\end{tabular}




\begin{tabular}{|c|c|c|c|c|}
\hline Suzuki et al., 2012 & \begin{tabular}{|ll} 
a. & Cross-sectional \\
b. & Retrospective chart \\
& review (pre-surgical \\
& data) \& telephone \\
& interview (post- \\
& surgical data)
\end{tabular} & $\begin{array}{ll}\text { a. } & \text { n=51 } \\
\text { b. } & \text { Patients were recruited who } \\
& \text { completed pre-operative } \\
& \text { psychological evaluation at } \\
& \text { Bringham and Women's } \\
& \text { Hospital and underwent } \\
& \text { bariatric surgery between } \\
& 2004 \text { and } 2007 \\
\text { c. } & \geq 2 \text { years post-surgery; } \geq 18 \\
& \text { years old } \\
\text { d. } & 460 \text { surveys mailed to } \\
& \text { patients; } 51 \text { agreed to } \\
& \text { participate (11.1\%) } \\
\text { e. } & \text { N/A } \\
\text { f. } & \text { RYGB 54.9\%, LAGB 45.1\% } \\
\text { g. } & \text { mean age= 51 (SD=8.7; 33- } \\
& 68) \\
\text { h. } & 86.3 \% \text { female } \\
\text { i. } & \text { N.D. }\end{array}$ & 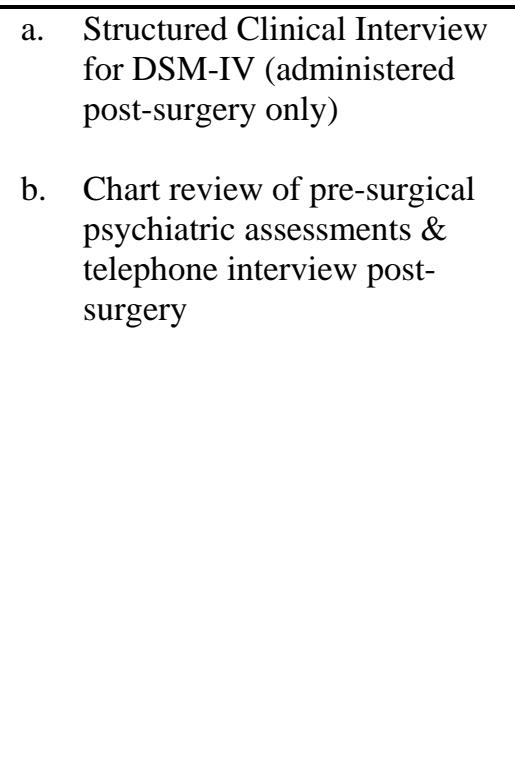 & 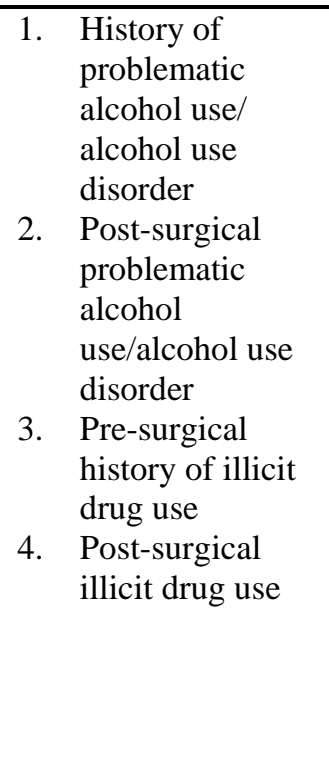 \\
\hline Odom et al., 2010 & 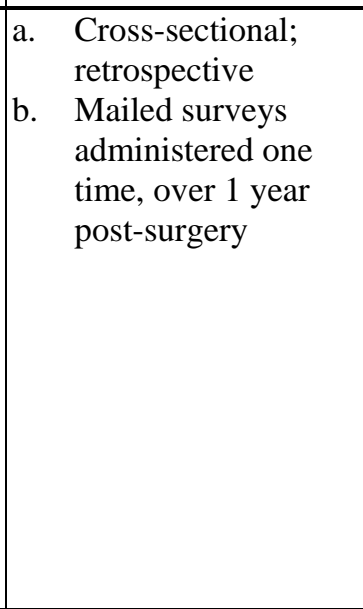 & $\begin{array}{ll}\text { a. } & \text { n=203 } \\
\text { b. } & \text { Surveys mailed to bariatric } \\
& \text { surgery patients identified } \\
& \text { through patient database from } \\
& \text { one hospital. } \\
\text { c. } & \text { N.D. } \\
\text { d. } & 1,117 \text { surveys mailed, } 24.8 \% \\
& \text { returned (n=278), 203 } \\
& \text { included in final analysis } \\
\text { e. } & 100 \% \text { RYGB } \\
\text { f. } & \text { Mean age = 50.6 (+/ } 9.8 \\
& \text { years) } \\
\text { g. } & 85 \% \text { female } \\
\text { h. } & 71.9 \% \text { white } \\
\text { i. } & \text { N.D. }\end{array}$ & 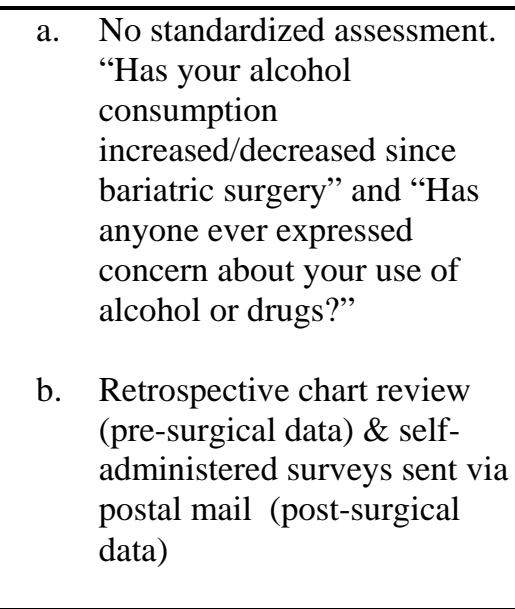 & 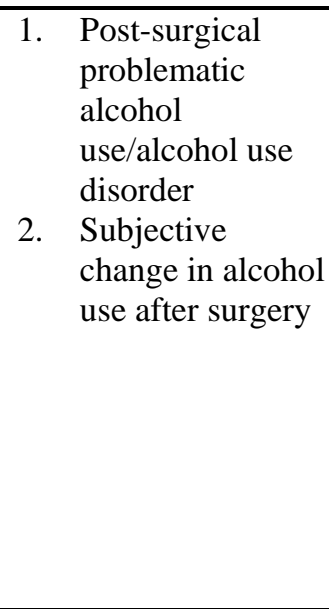 \\
\hline
\end{tabular}




\begin{tabular}{|c|c|c|c|c|c|}
\hline Adams et al., 2012 & $\begin{array}{ll}\text { a. } & \text { Medical record } \\
& \text { review } \\
\text { b. } & \text { Review of medical } \\
& \text { records collected } \\
& \text { pre-surgery, } 6,12, \\
& \text { and } 24 \text { months post- } \\
& \text { surgery }\end{array}$ & $\begin{array}{ll}\text { a. } & \mathrm{n}=61 \\
\text { b. } & \text { Chart review of veterans who } \\
& \text { underwent bariatric surgery } \\
& \text { from one VA facility between } \\
& 2003 \text { and 2008. } \\
\text { c. } & \text { Met national VA } \\
& \text { requirements for bariatric } \\
& \text { surgery } \\
\text { d. } & \text { N/A } \\
\text { e. } & \text { N/A } \\
\text { f. } & 59 \% \text { AGB, 41\% RYGB } \\
\text { g. } & \text { Mean age = 48 +/-7.3; 35-60) } \\
\text { h. } & 67 \%=\text { male } \\
\text { i. } & 70 \%=\text { white; } 28.3 \% \text { African } \\
& \text { American }\end{array}$ & & $\begin{array}{l}\text { AUDIT-C for alcohol use } \\
\text { diagnoses; DSM-IV based } \\
\text { diagnosis made by licensed } \\
\text { clinical psychologist or } \\
\text { healthcare provider for drug use } \\
\text { disorder( pre-surgery), and } \\
\text { urine screen (post-surgery) } \\
\text { Medical chart review }\end{array}$ & 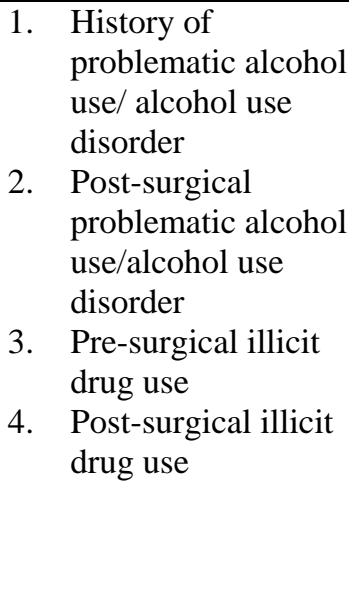 \\
\hline
\end{tabular}




\begin{tabular}{|c|c|c|c|c|c|}
\hline Tedesco et al., 2013 & \begin{tabular}{|ll} 
a. & Medical record \\
review \\
b. & Review of medical \\
& records collected pre- \\
& surgery and 2 weeks, \\
& 2 months, 6 months, \\
and 12 months post- \\
surgery
\end{tabular} & $\begin{array}{ll}\text { a. } & \begin{array}{l}\mathrm{n}=205 ; \mathrm{n}=74 \text { with a pre- } \\
\text { surgical history of substance } \\
\text { abuse (alcohol and drugs; SA }\end{array} \\
& \text { group), } \mathrm{n}=131 \text { with no } \\
& \text { substance abuse history (NA } \\
\text { group) } & \\
\text { b. } & \text { Veterans who had undergone } \\
& \text { bariatric surgery at the Palo } \\
& \text { Alto VA from 2002 and } 2011 \\
& \text { at one Veterans Affairs } \\
& \text { hospital; identified through } \\
& \text { database review } \\
\text { c. } & \text { N/A } \\
\text { d. } & \text { N/A } \\
\text { e. N/A } \\
\text { f. } & \text { 11.2\% AGB ( } \mathrm{n}=23 \text { ); 35\% } \\
& \text { ASG (n=72); 53.7\% RYGB } \\
\text { (n=110) } \\
\text { g. } & \text { Mean age = 51.5 } \\
\text { h. } & \text { SA group =86.5\% male; NA } \\
& \text { group =73.3\% male } \\
\text { i. } & \text { N.D. }\end{array}$ & & $\begin{array}{l}\text { Diagnosis made by mental } \\
\text { health professional based on } \\
\text { DSM-IV-TR criteria } \\
\text { Medical chart review; } \\
\text { participants were screened for } \\
\text { alcohol and substance abuse as } \\
\text { a part of their regular post-op } \\
\text { visits }\end{array}$ & 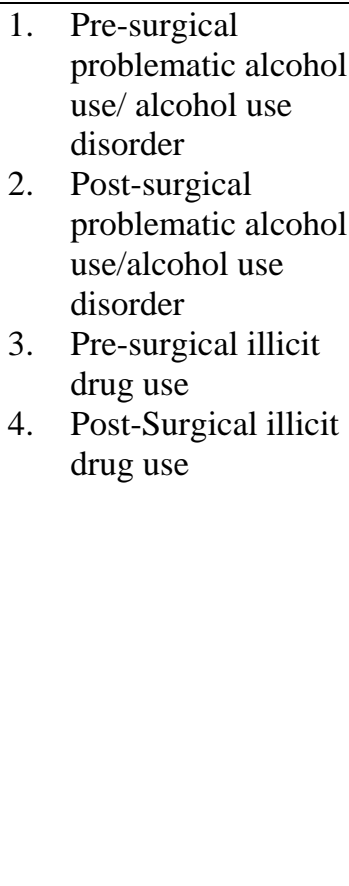 \\
\hline
\end{tabular}




\begin{tabular}{|c|c|c|c|c|}
\hline Saules et al., 2010 & $\begin{array}{l}\text { a. Medical record review } \\
\text { b. Retrospective review } \\
\text { of electronic medical } \\
\text { records }\end{array}$ & $\begin{array}{l}\text { a. Review of 7,199 medical } \\
\text { records } \\
\text { b. Review of medical records of } \\
\text { patients admitted to a drug and } \\
\text { alcohol treatment program } \\
\text { c. Admitted to drug and alcohol } \\
\text { treatment program from April 16, } \\
2006 \text { to May 31, } 2009 \text { and have a } \\
\text { history of WLS. } \\
\text { d. N/A } \\
\text { e. N/A } \\
\text { f. N.D. } \\
\text { g. Mean age }=44.7(S D=9.2) \\
\text { h. } 70.4 \% \text { female } \\
\text { i. } 81.5 \% \text { white }\end{array}$ & $\begin{array}{ll}\text { a. } & \text { N.D. } \\
\text { b. } & \text { Chart Review of substance } \\
& \text { abuse and alcohol abuse } \\
& \text { diagnoses among WLS patients }\end{array}$ & $\begin{array}{ll}\text { 1. } & \begin{array}{l}\text { Prevalence of patients } \\
\text { admitted to a } \\
\text { substance use } \\
\text { treatment facility that } \\
\text { have a history of }\end{array} \\
\text { WLS } \\
\text { 2. History of } \\
\text { problematic alcohol } \\
\text { use/alcohol use } \\
\text { disorder } \\
\text { 3. Post-surgical } \\
\text { problematic alcohol } \\
\text { use/alcohol use } \\
\text { disorder }\end{array}$ \\
\hline
\end{tabular}




\begin{tabular}{|c|c|c|c|c|c|}
\hline $\begin{array}{l}\text { Wiedemann } \\
\text { et al., } 2013\end{array}$ & $\begin{array}{l}\text { a. Medical record review } \\
\text { and semi-structured } \\
\text { interview } \\
\text { b. Retrospective review } \\
\text { of electronic medical } \\
\text { records, and interview } \\
\text { one time after surgery } \\
\text { with identified patients. }\end{array}$ & $\begin{array}{l}\text { a. Review of } 4,658 \text { medical } \\
\text { records, and interview of } 56 \\
\text { identified WLS patients. } \\
\text { b. Review of medical records of } \\
\text { patients admitted to a drug and } \\
\text { alcohol treatment program, and } \\
\text { recruitment to participate in an } \\
\text { interview. } \\
\text { c. Admitted to drug and alcohol } \\
\text { treatment program from July } 2009 \\
\text { to April 2011, have a history of } \\
\text { WLS, and agreed to an interview. } \\
\text { d. } 91.07 \% \text { ( } 51 \text { out of } 56 \text { patients) } \\
\text { e. N/A } \\
\text { f. } 90.6 \% \text { RYGB } \\
\text { g. Mean age =44.8 (SD=7.11) } \\
\text { h. } 71.4 \% \text { female } \\
\text { i. } 91.1 \% \text { white, }\end{array}$ & a. & $\begin{array}{l}\text { AUDIT-R for alcohol use, } \\
\text { questions developed by } \\
\text { investigators to assess illicit } \\
\text { drug use /alcohol use } \\
\text { trajectories (i.e. "Do you feel } \\
\text { like your problems with } \\
\text { alcohol/drugs began after you } \\
\text { had bariatric surgery? If yes, } \\
\text { please describe how you began } \\
\text { or increased your use of } \\
\text { alcohol/drugs, and how you } \\
\text { become concerned that it might } \\
\text { be a problem for you”), chart } \\
\text { review, self-reported } \\
\text { questionnaire, and semi- } \\
\text { structured interview } \\
\text { Self-reported questionnaire } \\
\text { (N.D. if self-administered or } \\
\text { interviewer administered) and } \\
\text { interviewer administered semi- } \\
\text { structured questionnaire. }\end{array}$ & $\begin{array}{ll}\text { 1. } & \begin{array}{l}\text { Prevalence of patients } \\
\text { admitted to a }\end{array} \\
\text { substance use } \\
\text { treatment facility that } \\
\text { have a history of } \\
\text { WLS } \\
\text { 2. History of } \\
\text { problematic alcohol } \\
\text { use/alcohol use } \\
\text { disorder } \\
\text { 3. Post-surgical } \\
\text { problematic alcohol } \\
\text { use/alcohol use } \\
\text { disorder } \\
\text { 4. Pre-surgical } \\
\text { substance use } \\
\text { disorder } \\
\text { 5. Post-surgical } \\
\text { substance use } \\
\text { disorder }\end{array}$ \\
\hline
\end{tabular}




\begin{tabular}{|c|c|c|c|c|}
\hline $\begin{array}{l}\text { Cuellar-Barboza et } \\
\text { al., } 2014\end{array}$ & $\begin{array}{|ll|}\text { a. } & \text { Medical record } \\
& \text { review } \\
\text { b. } & \text { Retrospective review } \\
& \text { of electronic medical } \\
& \text { records }\end{array}$ & $\begin{array}{l}\text { a. Review of } 823 \text { electronic } \\
\text { medical records (EMR) } \\
\text { b. Data extracted from EMRs of } \\
\text { patients treated at the Mayo Clinic } \\
\text { Addiction Treatment Program, } \\
\text { between June } 2004 \text { and July } 2012 \\
\text { c. Patients aged } 30 \text {-60 with a } \\
\text { primary treatment diagnosis of } \\
\text { AUD, and had the RYGB } \\
\text { procedure } \\
\text { c. N/A } \\
\text { d. N/A } \\
\text { E. N/A } \\
\text { f. } 100 \% \text { RYGB } \\
\text { g. Mean age of admission=46 } \\
\text { (1.3) } \\
\text { h. } 89.6 \% \text { female } \\
\text { i. } 92.7 \% \text { white }\end{array}$ & $\begin{array}{ll}\text { a. } & \text { Data extracted from clinical notes, } \\
& \text { reflecting clinical evaluation based } \\
\text { on DSM-V criteria.* } \\
\text { b. }\end{array}$ & 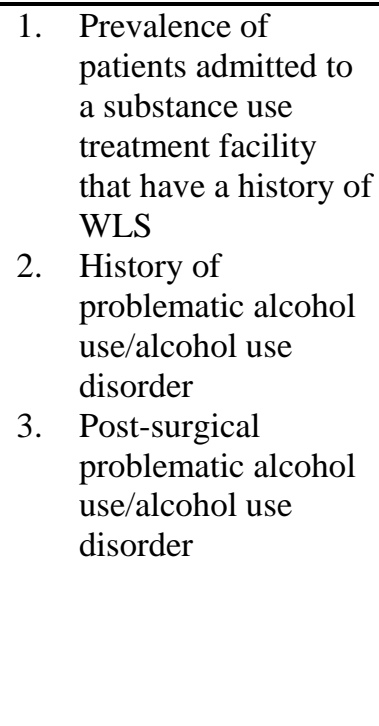 \\
\hline
\end{tabular}




\begin{tabular}{|c|c|c|c|c|}
\hline Ostlund et al., 2013 & \begin{tabular}{|ll} 
a. & Medical record \\
& review \\
b. & Review of all \\
& patients in Sweden \\
& who had bariatric \\
& surgery procedure \\
& from January 1, 1980 \\
& to December 31, \\
& 2006 via the Swedish \\
& Patient Registrar
\end{tabular} & $\begin{array}{ll}\text { a. } & \text { n= 11,115 } \\
\text { b. } & \text { Review of the nationwide } \\
& \text { dataset, the Swedish Patient } \\
& \text { Registrar, from January 1, } \\
& 1980 \text { to December 31, 2006 } \\
& \text { for all patients who had } \\
& \text { bariatric surgery. } \\
\text { c. } & \text { Bariatric surgery patients } \\
\text { d. } & \text { Patients who had more than 1 } \\
& \text { bariatric procedure } \\
\text { e. } & \text { N/A } \\
\text { f. } & \text { 37\% RYGB; 63\% AGB \& } \\
& \text { VBG } \\
\text { g. } & \text { RYGB cohort = 40.2 (10.1) } \\
& \text { AGB\&VBG cohort =40.0 } \\
& (10.4) \\
\text { h. } & \text { RYGB cohort, female = 75\%; } \\
& \text { AGB \&VBG cohort, female } \\
& =78 \% \\
\text { i. } & \text { N.D. (study conducted in } \\
& \text { Sweden) } \\
\end{array}$ & $\begin{array}{l}\text { a. Clinical diagnosis for substance } \\
\text { abuse or alcohol abuse (no other } \\
\text { information given) } \\
\text { b. Chart Review of substance abuse } \\
\text { and alcohol abuse diagnoses as } \\
\text { documented in the Swedish Patient } \\
\text { Register }\end{array}$ & $\begin{array}{l}\text { 1. Prevalence of patients } \\
\text { admitted to a } \\
\text { substance use } \\
\text { treatment facility that } \\
\text { have a history of } \\
\text { WLS }\end{array}$ \\
\hline Omalu et al., 2007 & \begin{tabular}{|ll} 
a. & Retrospective record \\
& review \\
b. & Chart review of \\
& Pennsylvania State \\
& health care database \\
& and death records to \\
& examine death rate \\
& and causes of death \\
& for all Pennsylvania \\
& residents who had \\
& bariatric surgery from \\
1995-2005
\end{tabular} & $\begin{array}{ll}\text { a. } & \mathrm{n}=16,683 \text { total surgeries; } \\
& 440 \text { deaths } \\
\text { b. } & \text { investigation of death rates } \\
& \text { and causes of death for all } \\
& \text { Pennsylvania residents who } \\
& \text { had bariatric surgery from } \\
& 1995-2005 \\
\text { c. } & \text { N/A } \\
\text { d. } & \text { N/A } \\
\text { e. } & \text { N/A } \\
\text { f. } & \text { N.D. } \\
\text { g. } & 48=\text { Mean age at time of } \\
& \text { surgery } \\
\text { h. } & 82.3 \% \text { female }(n=13,734) \\
\text { i. } & 81 \% \text { white }(n=13,504)\end{array}$ & $\begin{array}{ll}\text { a. } & \text { Review of death records } \\
\text { b. } & \text { Chart review of death records } \\
\text { obtained from the Division of Vital } \\
\text { Records, Pennsylvania State } \\
\text { Department of Health investigating } \\
\text { drug overdose as cause of death }\end{array}$ & $\begin{array}{ll}\text { 1. } & \text { Post-surgical illicit } \\
\text { drug use }\end{array}$ \\
\hline
\end{tabular}




\begin{tabular}{|c|c|c|c|c|}
\hline $\begin{array}{l}\text { Reslan, Saules, } \\
\text { Greenwald, \& } \\
\text { Schuh, } 2014\end{array}$ & \begin{tabular}{|ll} 
a. & Cross-sectional; \\
retrospective \\
b. & One time
\end{tabular} & $\begin{array}{l}\text { a. } \mathrm{n}=141 \\
\text { b. Participants who previously } \\
\text { participated in prior bariatric } \\
\text { research or bariatric support } \\
\text { groups were recruited. } \\
\text { c. } \text { RYGB patients, at least } 24 \\
\text { months post-operative; not } \\
\text { currently pregnant } \\
\text { d. N.D. } \\
\text { e. } N / A \\
\text { f. } 100 \% \text { RYGB } \\
\text { g. mean age }=53(\mathrm{SD}=10.33) \\
\text { h. } 79 \% \text { female }(\mathrm{n}=112) \\
\text { i. } 93 \% \text { white }(=130)\end{array}$ & $\begin{array}{l}\text { a. Michigan assessment-screening test } \\
\text { for alcohol and drugs (MAST/AD) and } \\
\text { the Alcohol, Smoking and Substance } \\
\text { Involvement Screening Test(ASSIST) } \\
\text { b. Self-reported; web-based survey or } \\
\text { mailed survey }\end{array}$ & $\begin{array}{l}\text { Pre-surgical history of } \\
\text { substance use disorders } \\
\text { (alcohol and illicit drug } \\
\text { use not distinguished). } \\
\text { 2. Post-surgical substance } \\
\text { use disorders (alcohol and } \\
\text { illicit drug use not } \\
\text { distinguished). }\end{array}$ \\
\hline $\begin{array}{l}\text { Ivezaj, Saules, \& } \\
\text { Schuh, } 2014\end{array}$ & \begin{tabular}{|ll} 
a. & Cross-sectional; \\
retrospective \\
b. & One time
\end{tabular} & $\begin{array}{l}\text { a. } \mathrm{n}=143 \\
\text { b. Participants recruited } \\
\text { through St. Vincent Caramel } \\
\text { Hospital's Bariatric Center of } \\
\text { Excellence and through an } \\
\text { online support group } \\
\text { c. Post-surgical RYGB } \\
\text { patients } \\
\text { d. } \text { N.D. } \\
\text { e. N/A } \\
\text { f. } 100 \% \text { RYGB } \\
\text { g. mean age= } 48.97(\mathrm{SD}=10.5) \\
\text { h. } 83.9 \% \text { female }(\mathrm{n}=120) \\
\text { i. } 94.4 \% \text { white }(\mathrm{n}=135)\end{array}$ & $\begin{array}{l}\text { a. Michigan assessment-screening test } \\
\text { for alcohol and drugs (MAST/AD) } \\
\text { b. Self-reported; web-based survey }\end{array}$ & $\begin{array}{l}\text { 1. Pre-surgical history of } \\
\text { substance use disorders } \\
\text { (alcohol and illicit drug } \\
\text { use not distinguished). } \\
\text { 2. Post-surgical substance } \\
\text { misuse (alcohol and illicit } \\
\text { drug use not } \\
\text { distinguished). }\end{array}$ \\
\hline
\end{tabular}




\begin{tabular}{|c|c|c|c|c|}
\hline Fowler et al., 2014 & $\begin{array}{ll}\text { a. } & \text { Cross-sectional; } \\
\text { retrospective } \\
\text { b. }\end{array}$ & $\begin{array}{ll}\text { a. } & \mathrm{n}=154 \\
\text { b. } & \text { N/A Secondary data analysis } \\
& \text { of a bariatric surgery patient } \\
& \text { database } \\
\text { c. } & \text { N/A } \\
\text { d. } & \text { N/A } \\
\text { e. } & \text { N/A } \\
\text { f. } & 92.9 \% \text { RYGB } \\
\text { g. } & \text { Mean age }=48.7(\mathrm{SD}=10.8) \\
\text { h. } & 88.4 \% \text { female } \\
\text { i. } & 94.2 \% \text { white } \\
\end{array}$ & $\begin{array}{ll}\text { a. } & \begin{array}{l}\text { Michigan assessment-screening test } \\
\text { for alcohol and drugs (MAST/AD) } \\
\text { and Yale Food Addiction Scale }\end{array} \\
\text { (YFAS) } \\
\text { b. N.D. }\end{array}$ & $\begin{array}{l}\text { 1. Pre-surgical substance } \\
\text { use disorders (alcohol and } \\
\text { illicit drug use not } \\
\text { distinguished) as related } \\
\text { to pre-surgical } \\
\text { endorsement of } \\
\text { problematic intake of } \\
\text { certain foods. }\end{array}$ \\
\hline Fogger et al., 2012 & $\begin{array}{ll}\text { a. } & \text { Cross-sectional } \\
\text { b. } & \text { One-time; post- } \\
& \text { surgery }\end{array}$ & $\begin{array}{ll}\text { a. } & \text { Total =173; bariatric } \\
& \text { surgery patients =25 } \\
\text { b. } & \text { Analysis of a sub-group of } \\
\text { bariatric surgery patients } \\
\text { among a study investigating } \\
\text { the efficacy of a state- } \\
\text { monitoring program for } \\
\text { nurses with addictions i } \\
\text { c. } & \text { N/A } \\
\text { d. } & 382 \text { surveys mailed; } 173 \\
& \text { returned } \\
\text { e. } & \text { N/A } \\
\text { f. } & \text { N.D. } \\
\text { g. } & \text { N.D. } \\
\text { h. } & \text { Bariatric surgery subgroup: } \\
& \text { women=24 } \\
\text { i. } & \text { N.D. }\end{array}$ & $\begin{array}{l}\text { a. Three qualitative questions } \\
\text { regarding bariatric surgery: (1) } \\
\text { Have you ever had bariatric surgery, } \\
\text { (2) Did your problem with } \\
\text { substances begin after surgery? (3) } \\
\text { If you had a substance problem } \\
\text { prior to surgery did it change after } \\
\text { bariatric surgery? } \\
\text { b. Mailed survey }\end{array}$ & $\begin{array}{l}\text { Prevalence of nurses } \\
\text { who underwent } \\
\text { bariatric surgery } \\
\text { participating in a state } \\
\text { monitoring program } \\
\text { for nurses with } \\
\text { addiction }\end{array}$ \\
\hline
\end{tabular}

- $\quad$ Key: N.D. = no data; N/A= not applicable; RYGB = Roux en y gastric bypass; ASG = sleeve gastrectomy; AGB= Adjustable Gastric Band; VBG = vertical banded gastroplasty (stomach stapling); $\mathrm{SD}=$ standard deviation 
TABLE 3

Summary of Results Among Studies Examining Alcohol and Illicit Drug Use in Post-Bariatric Populations

\begin{tabular}{|c|c|c|}
\hline Outcome Measured & Reference & Results \\
\hline $\begin{array}{l}\text { Post-surgical } \\
\text { problematic alcohol use/ } \\
\text { alcohol use disorders } \\
\text { (AUDs) } \\
\text { A. Main Finding(s) } \\
\text { B. Data stratified } \\
\text { by surgery type } \\
\text { C. Predictors/ Risk } \\
\text { Factors }\end{array}$ & Svensson et al., 2013 & $\begin{array}{l}\text { A. 93.1\% of the surgery group and } 96 \% \text { of the non-surgical control group } \\
\text { reported low risk alcohol consumption at all follow up points [risk } \\
\text { defined by World Health Organization (WHO), less than } 3 \text { drinks per } \\
\text { day for men and } 1.5 \text { drinks per day for women ] } \\
\begin{array}{r}\text { B. Compared to non-surgical control group: } \\
\text { RYGB patients were at an increased risk for demonstrating } \\
\text { at least medium risk alcohol intake behaviors (adjusted } \\
\text { hazard ratio [adjHR] =2.69), an increased risk of alcohol } \\
\text { abuse diagnosis (adjHR=4.97), and self-reported alcohol } \\
\text { problems (adjHR=5.91) } \\
\text { VBG patients also demonstrated increased risk for alcohol } \\
\text { consumption at least at the WHO medium risk level } \\
\text { (adjHR=1.52), an increased risk of alcohol abuse diagnoses } \\
\text { (adjHR = 2.23), and self-reported alcohol problems } \\
\text { (adjHR=2.30). } \\
\text { AGB patients did not demonstrate higher alcohol risk } \\
\text { behavior compared to controls. }\end{array}\end{array}$ \\
\hline
\end{tabular}




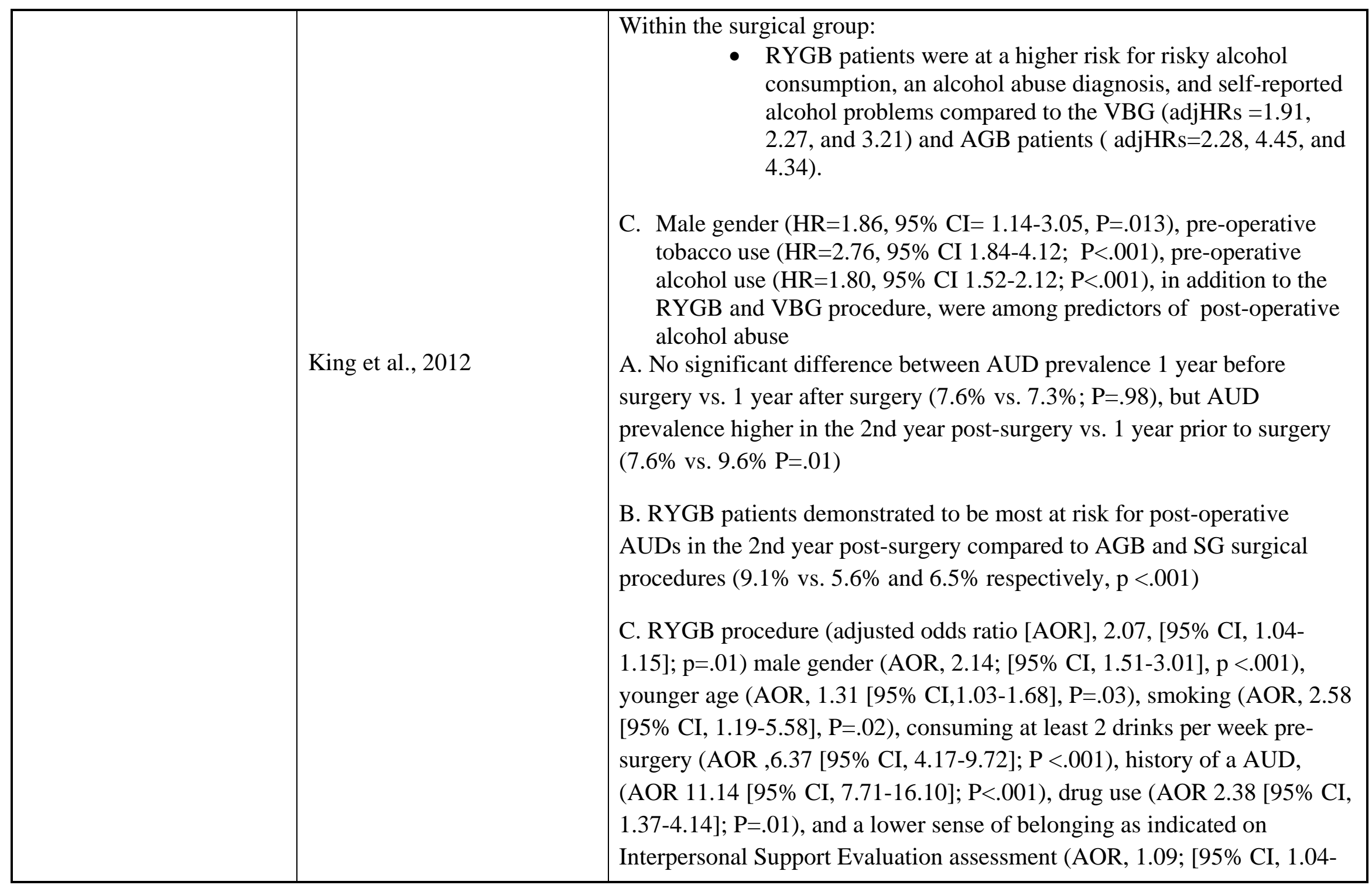




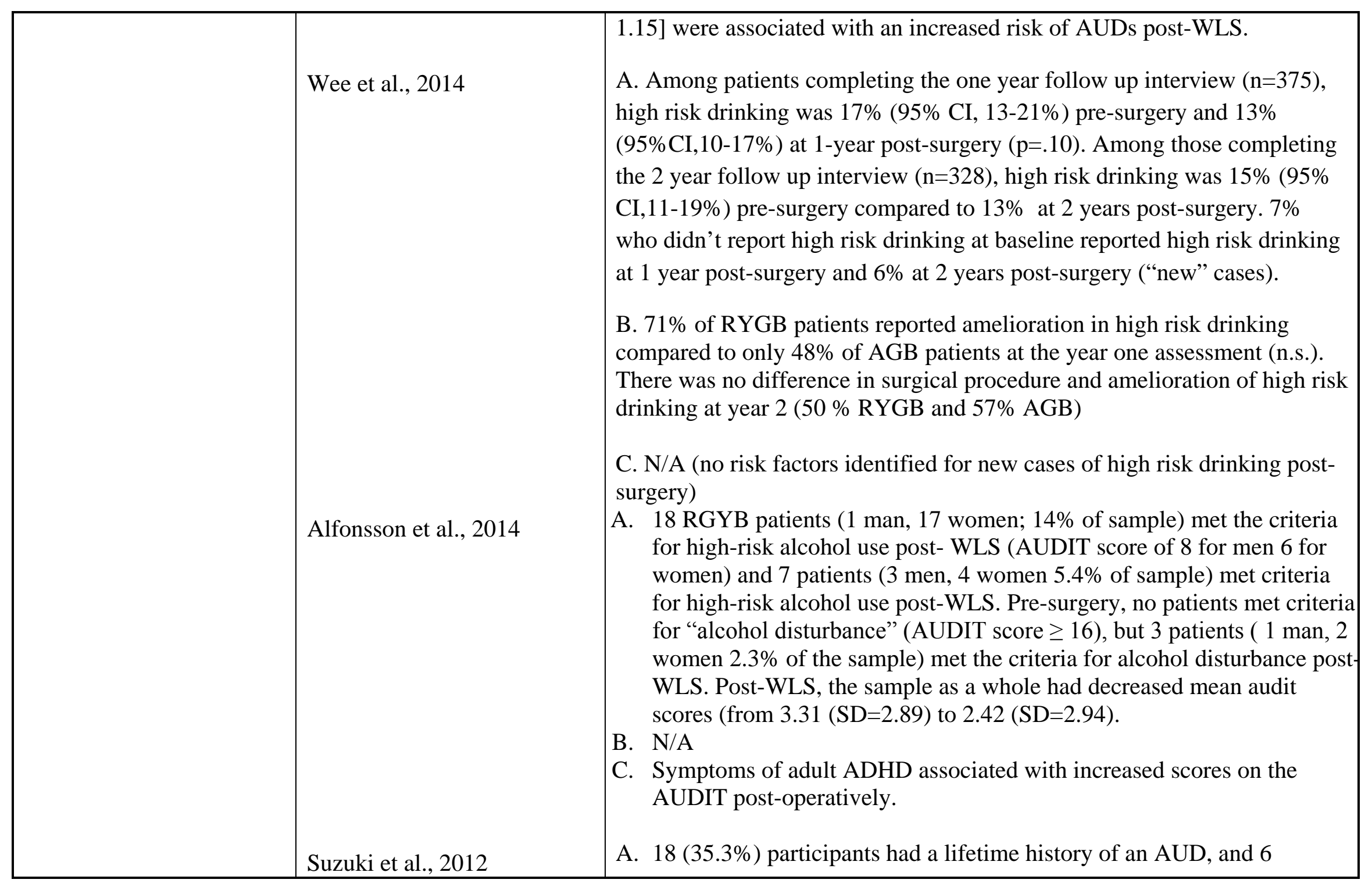




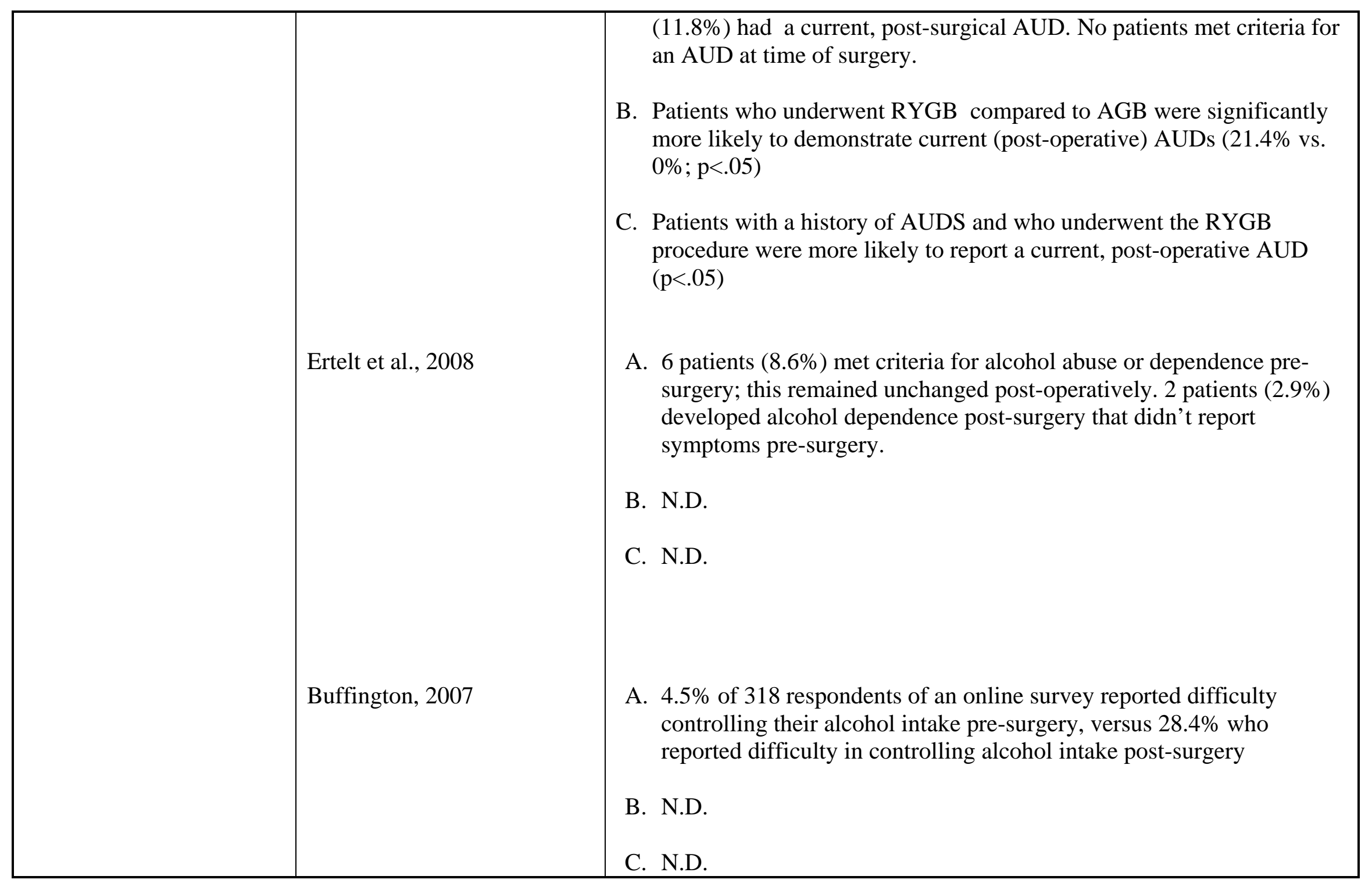




\begin{tabular}{|c|c|c|}
\hline & Saules et al., 2010 & $\begin{array}{l}\text { A. Between } 2-6 \% \text { of past } 3 \text { year admissions at a drug treatment facility } \\
\text { were bariatric surgery patients, based on medical chart review. } 62.3 \% \\
\text { of these patients sought treatment for alcohol use, and } 9.4 \% \text { sought } \\
\text { treatment for alcohol use plus illicit drug use. The remainder sought } \\
\text { treatment for illicit drug use. } 61.9 \% \text { of alcohol users reported } \\
\text { engaging in heavy use before their surgery, and } 43.4 \% \text { of patients did } \\
\text { not engage in heavy alcohol or illicit drug use until after surgery, } \\
\text { representing new onset cases } \\
\text { B. N.D. } \\
\text { C. N.D. }\end{array}$ \\
\hline & (1) & $\begin{array}{l}\text { A. } 2.8 \% \text { of } 4,658 \text { patients in a substance use treatment facility were WLS } \\
\text { patients, based on review of electronic records. WLS patients were } \\
\text { more likely to have an AUD diagnosis compared to non-WLS controls } \\
\left(\mathrm{x}^{2}(1, n=4658)=7.41, \mathrm{P}<0.01\right) \text {. Of the WLS patient subset } 56 \text { were } \\
\text { recruited to undergo a qualitative interview, } 60 \% \text { were classified as } \\
\text { developing a SUD post-surgery, and } 40 \% \text { had a pre-operative history } \\
\text { of a SUD. WLS patients took } 1.6 \text { years }(1.62) \text { to become concerned } \\
\text { with SUD. } \\
\text { B. N.D. } \\
\text { C. N.D. }\end{array}$ \\
\hline & Cuellar-Barboza, et al., 2014 & $\begin{array}{l}\text { A. } 4.9 \%(\mathrm{n}=41) \text { of } 823 \text { patients admitted to an AUD treatment program } \\
\text { previously had the RYGB procedure. Of the WLS subset } 61 \%(\mathrm{n}=25) \\
\text { met criteria for an AUD after surgery, and 39\% met criteria for an } \\
\text { AUD prior t.o surgery. } 17 \% \text { reported not consuming alcohol until after } \\
\text { surgery ( } \mathrm{n}=7) \text {. RYGB patients met criteria for an AUD } 3.1 \text { years } \\
\text { (S.D.=.5) post-operatively and sought treatment } 5.4 \text { (S.D.=.3) years } \\
\text { post-operatively. }\end{array}$ \\
\hline
\end{tabular}




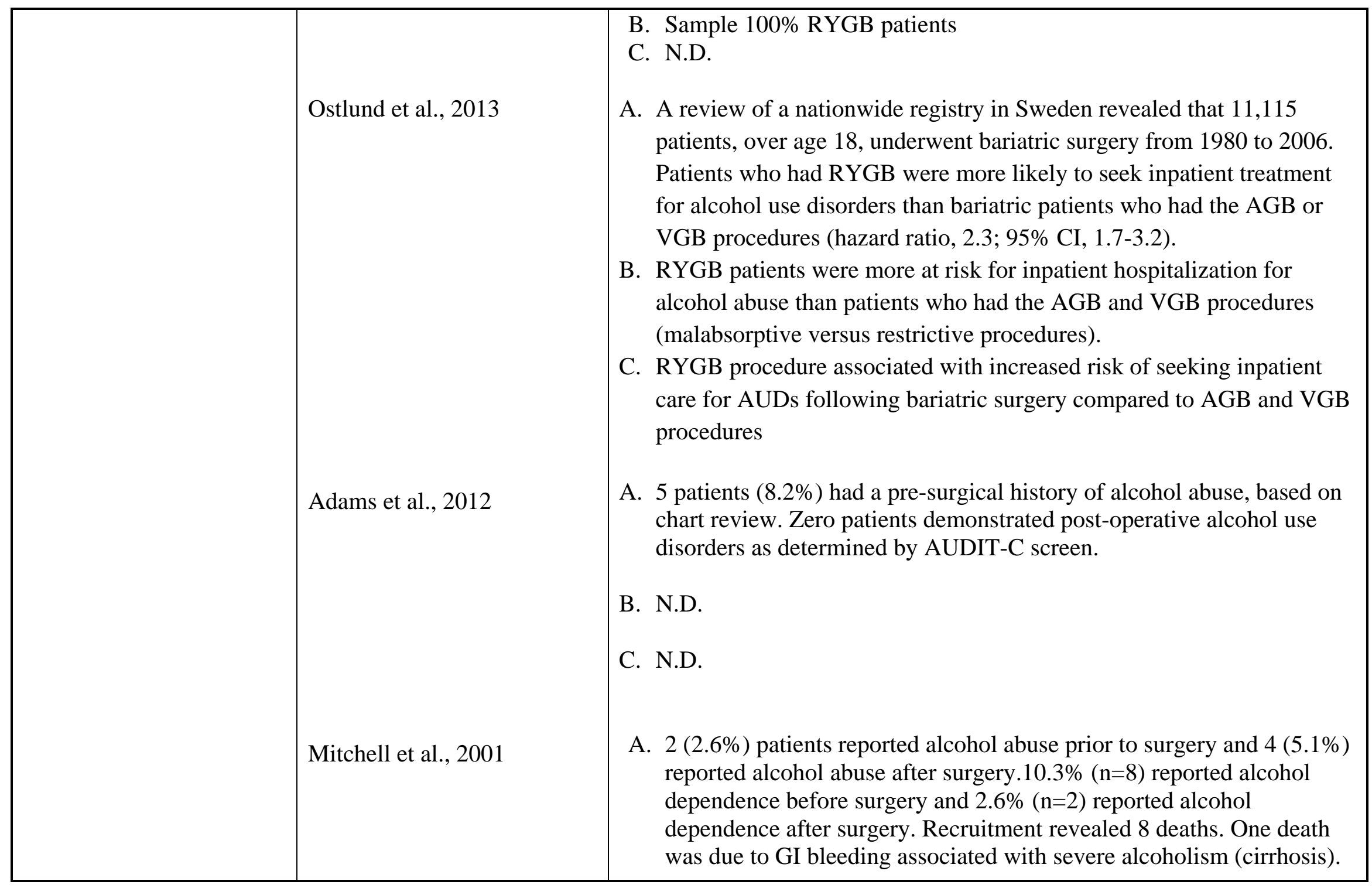




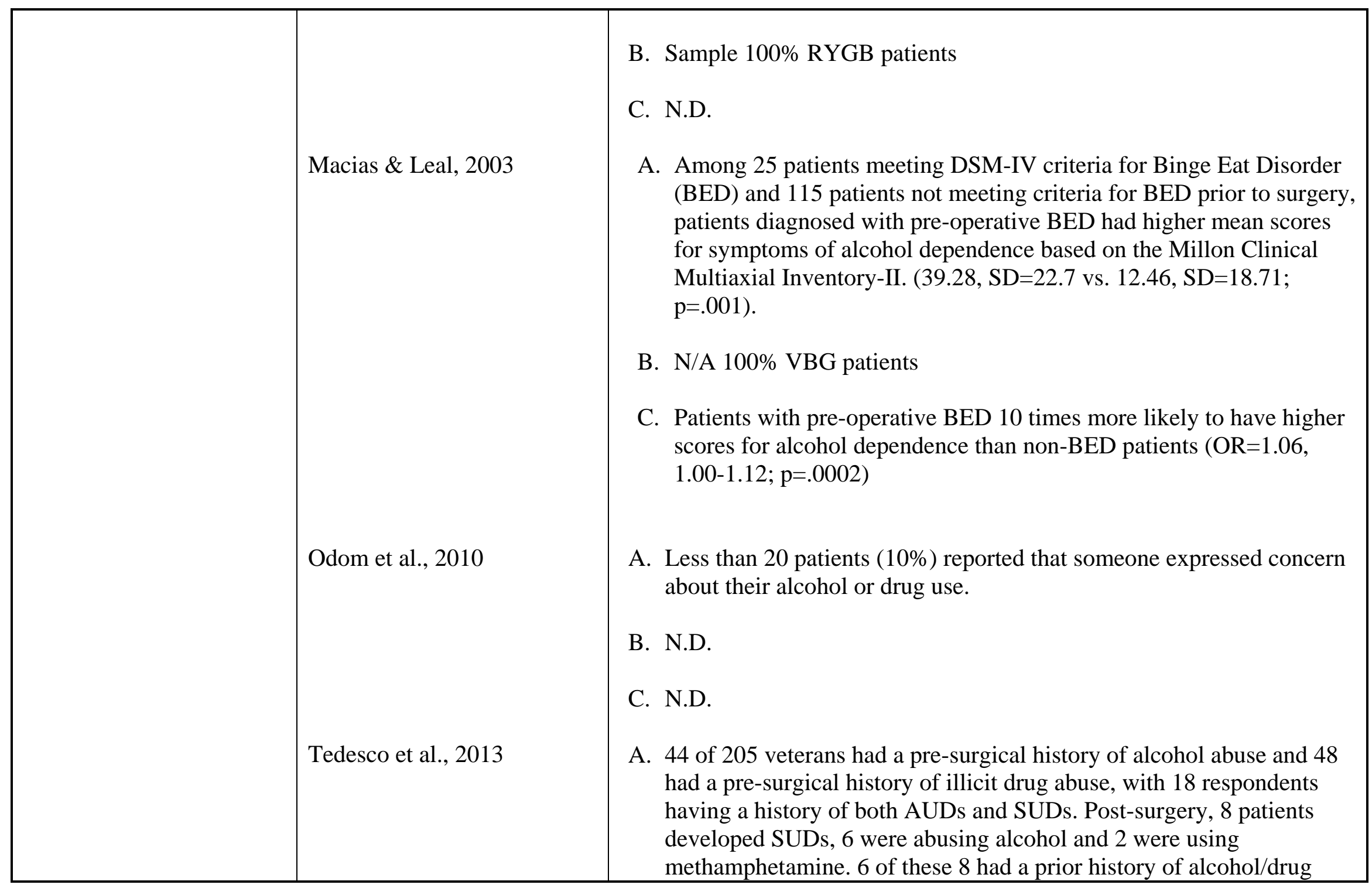




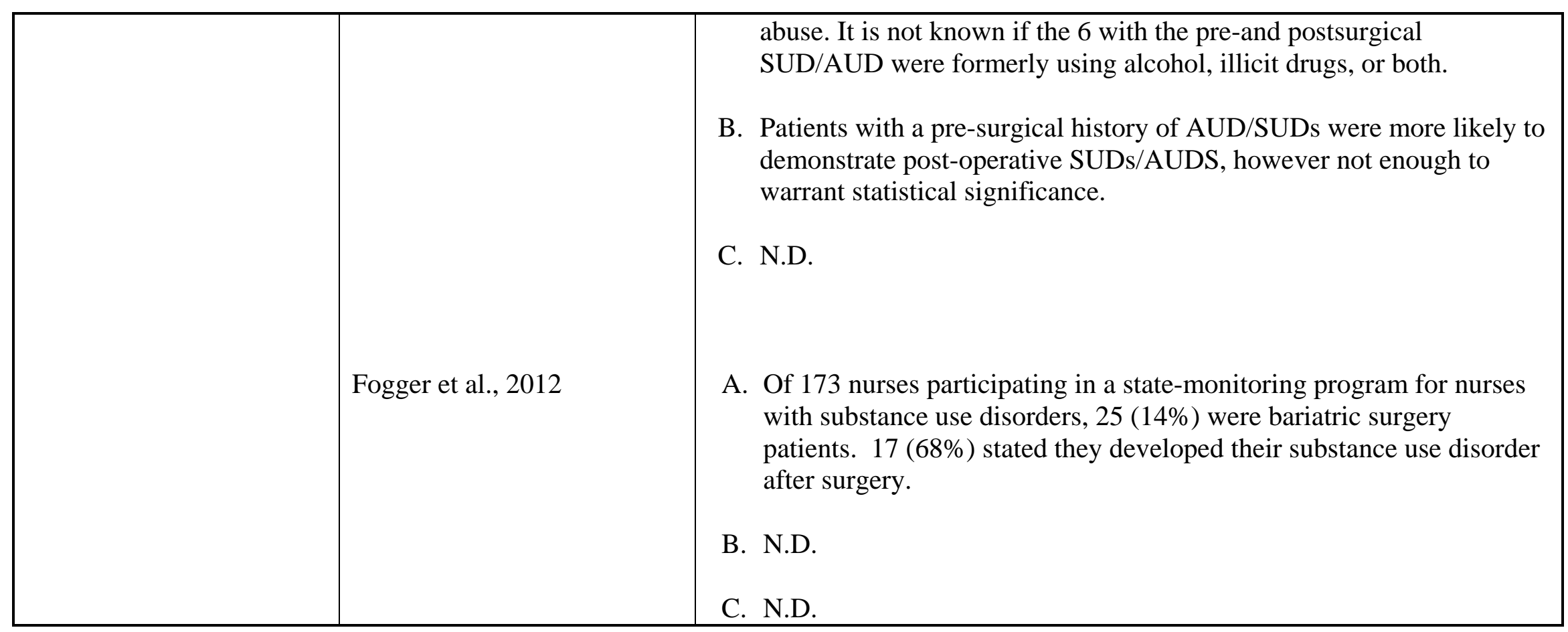




\section{Post-surgical alcohol use/frequency of alcohol}

use

A. Main Finding(s)

B. Data stratified by surgery type

C. Predictors/ Risk Factors
Conason et al., 2012

Lent et al., 2013

Odom et al., 2010
A. Significant decrease in alcohol use found from baseline (pre-surgery) to 1 month $(\mathrm{p}<.001)$ and 3 months $(\mathrm{p}=.009)$ post-surgery. Significant increase in frequency of alcohol use post-surgery was found: from 1 month to 12 months ( $<<.001), 1$ month to 24 months ( $p$ $<.001)$, 3 months to 12 months ( $\mathrm{p}=.016)$, and from 3 to 24 months $(\mathrm{p}<.001)$, and 6 months to 24 months ( $\mathrm{p}=.001)$.

B. RYGB patients reported significant increase in frequency of alcohol use from baseline to 24 months $(\mathrm{p}=.011)$. No significant increases found in LAGB patients

C. N.D.

A. Significant decrease among patients reporting alcohol use from before surgery $(n=112 ; 72.3 \%)$ to after surgery $(n=98 ; 63.2 \% ; p=.026) .24$ (21.4\%) of the 112 respondents who used alcohol before surgery reported no alcohol use post-surgery.10 (23.2\%) of the 43 participants who reported no alcohol use in the year prior to surgery reported alcohol use after surgery. Post-surgery, 54.8\% $(n=85)$ classified themselves as consuming 1-4 drinks on a typical drinking occasion, and $3.2 \%(n=5)$ classified themselves as drinking over 5 drinks on a typical occasion. The majority of post-operative patients reported drinking monthly or less ( $n=60 ; 38.7 \%)$, followed by $2-4$ times per month ( $n=27 ; 17.4 \%), 2-3$ times per week $(n=5,3.2 \%), 4-5$ times per week ( $n=5,3.2 \%)$, a over 5 times per week $(n=1,0.6 \%)$.

B. Sample $100 \%$ RYGB patients

C. Quantity of alcohol use prior to surgery was the strongest predictor of post-surgical alcohol use (EXP [B] = 6.36, 95\% CI [3.09-13.08], $p$ $<.001)$. Older age decreased the likelihood of post-surgical alcohol use (EXP [B]=.954, 95\% CI [.919-.990], $p=.013$ ). High post-surgical alcohol consumption was associated with higher pre-operative BMI $(\mathrm{EXP}[\mathrm{B}]=1.23,95 \% \mathrm{CI}[1.05-1.44], p=.01)$.

A. $25(9.1 \%)$ patients identified an increase in alcohol use since their surgery; 53(19.1\%) reported a decrease in alcohol use,83 (30.1\%) reported no change in alcohol use pre-surgery and; 115 (41.7\%) 187 never used alcohol.

B. Sample $100 \%$ RYGB patients

C. N.D 


\begin{tabular}{|c|c|c|}
\hline $\begin{array}{l}\text { Post-surgical change in } \\
\text { sensitivity to alcohol } \\
\text { (subjective account) } \\
\text { A. Main Finding(s) } \\
\text { B. Surgery Type }\end{array}$ & Buffington, 2007 & $\begin{array}{l}\text { A. } 38 \text { patients (54.3\%) indicated a change in response to alcohol post- } \\
\text { surgery; of these } 24 \text { ( } 34.3 \% \text { ) patients reported quicker intoxication, } \\
\text { and } 14(20 \%) \text { reported feeling intoxicated after drinking lower } \\
\text { quantities of alcohol compared to before surgery. No one indicated } \\
\text { that it took them longer to reach intoxication post-surgery or took } \\
\text { more quantities of alcohol post-surgery to become intoxicated. } \\
\text { B. Sample } 100 \% \text { RYGB patients } \\
\text { A. } 83 \% \text { of } 318 \text { respondents reported consuming alcohol after surgery. } \\
\text { 84\% of the sample who reported having one or more beverages } \\
\text { weekly, reported increased sensitivity to alcohol post-surgery. } 44 \% \\
\text { said they felt a physical response after only a few sips, and } 45 \% \text { felt } \\
\text { the effects after one drink. } 29 \% \text { felt the effects of alcohol for a longer } \\
\text { period of time compared to before surgery. } \\
\text { B. } 100 \% \text { RYGB }\end{array}$ \\
\hline \multirow{3}{*}{$\begin{array}{l}\text { Post-surgical illicit drug } \\
\text { use }\end{array}$} & Mitchell et al., 2001 & $\begin{array}{l}\text { A. } 3.8 \%(n=3) \text { reported drug abuse pre-surgery, and } 1.3 \%(n=1) \text { reported } \\
\text { drug abuse after surgery (n.s.). }\end{array}$ \\
\hline & Suzuki et al., 2012 & $\begin{array}{l}\text { A. } 13.7 \%(n=7) \text { of participants demonstrated a lifetime history for a drug } \\
\text { use disorder, no participants }(n=0) \text { demonstrated to have a current, } \\
\text { post-surgical drug use disorder. }\end{array}$ \\
\hline & Adams et al., 2012 & A. $3(5 \%)$ of patients reported a pre-surgical history of a drug use \\
\hline
\end{tabular}




\begin{tabular}{|c|c|c|}
\hline & $\begin{array}{l}\text { Tedesco et al., } 2013 \\
\text { Omalu et al., } 2007\end{array}$ & $\begin{array}{l}\text { (cocaine) disorder diagnosis, but were not active drug users at the } \\
\text { time of their surgery. One (1.6\%) of these patients demonstrated to be } \\
\text { an active drug user post-surgery as revealed by a positive urine drug } \\
\text { screen test, indicating a relapse. } \\
\text { A. Of } 205 \text { patients, } 44(23.4 \%) \text { had a pre-surgical history of illicit drug } \\
\text { use, but were not believed to be active drug users at the time of their } \\
\text { surgery. Post-operatively } 2 \text { patients used methamphetamine, } \\
\text { indicating a drug use relapse post-surgery. } \\
\text { A. Of } 440 \text { deaths, } 14 \text { (3\%) were attributed to drug overdoses, as } \\
\text { indicated on the patient's death certificate. } 12 \text { of these deaths } \\
\text { occurred in women. }\end{array}$ \\
\hline $\begin{array}{l}\text { Post-surgical } \\
\text { “substance” abuse } \\
\text { (combined alcohol and } \\
\text { illicit drug use) } \\
\text { A. Main Finding(s) } \\
\text { B. Data stratified by } \\
\text { surgery type } \\
\text { C. Predictors/ Risk } \\
\text { Factors }\end{array}$ & Reslan et al., 2014 & $\begin{array}{l}\text { A. } 20 \text { participants }(14 \% \text {; } n=141) \text { reported probable post-operative } \\
\text { substance misuse, which was defined as a score of } 5 \text { or higher on the } \\
\text { MAST/AD. Tobacco, alcohol, and illicit drug use were not } \\
\text { distinguished. Of the subset reporting probable post-operative } \\
\text { substance misuse, } 14(70 \%) \text { did not report probable misuse prior to } \\
\text { surgery, suggesting new onset of probable substance misuse post- } \\
\text { surgery; } 6 \text { of the } 20 \text { reported both pre and post-operative probable } \\
\text { substance misuse, suggesting continued use post-surgery; and 6\% } \\
\text { (n=8) reported pre-operative probable substance misuse but not post- } \\
\text { operative substance misuse, suggesting recovery from substance } \\
\text { misuse post-surgery. } 80 \% \text { did not meet criteria for both pre-and post- } \\
\text { operative probable substance misuse } \\
\text { B. N/A, sample entirely RYGB patients. } \\
\text { C. Current (post-operative) probable substance misuse was associated } \\
\text { with younger age (M=46.70 SD=9.91 vs. } M=54.5 \text {, SD=10.15, }\end{array}$ \\
\hline
\end{tabular}




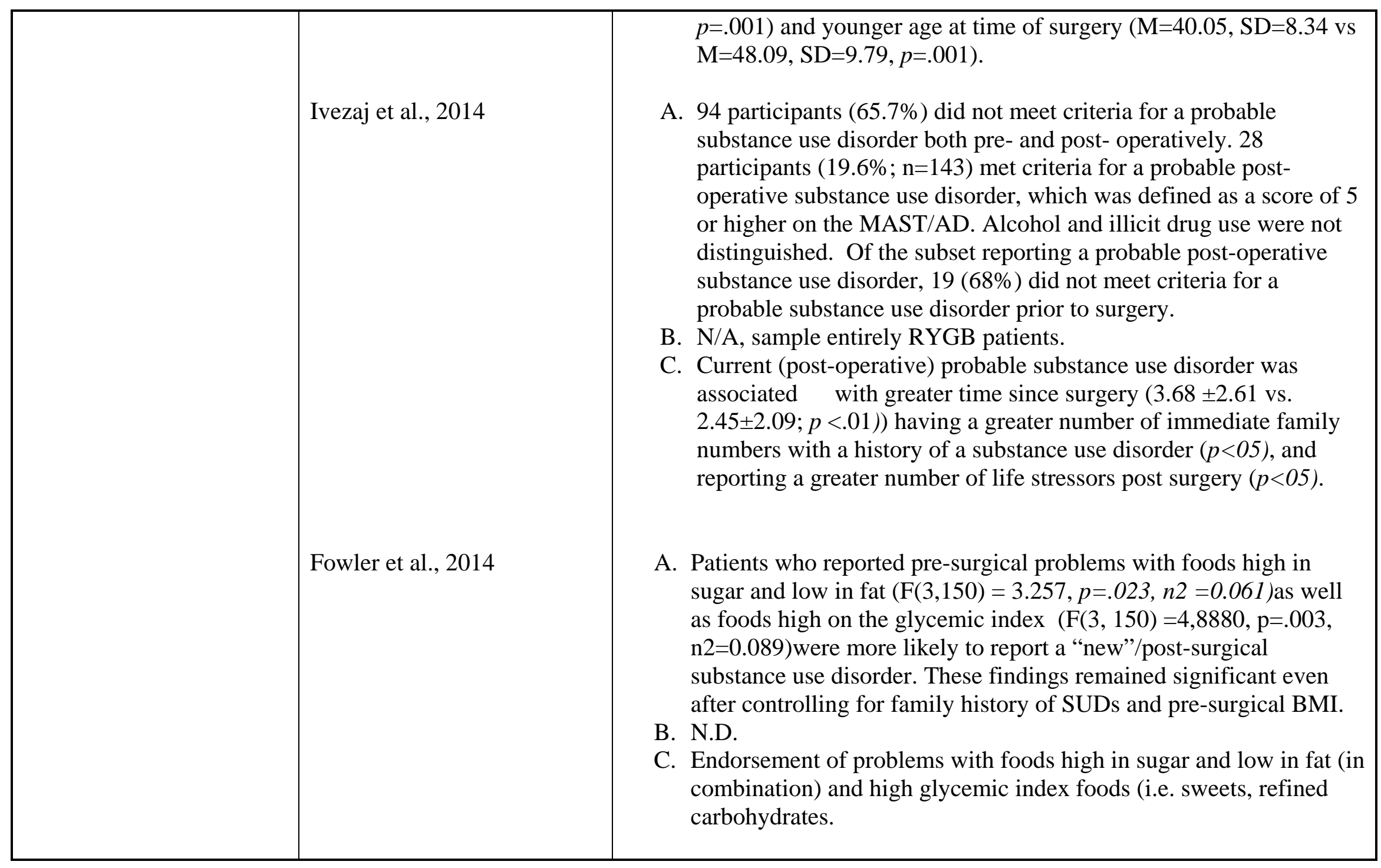




\title{
QUALITATIVE INTERVIEW SCHEDULE
}

\author{
ID: \\ Date: \\ Type of Surgery: \\ Date of Surgery: \\ Gender: \\ Age:
}

Thanks for taking the time to talk to me today. I'm going to ask you some questionsthere are no wrong or right answers. Your answers are you own, and will help me to know more about alcohol use and bariatric surgery. Ready to begin?

On-going prompts:

Can you give me an example of that?

Can you tell me a time when that happened?

Can you tell me a time when this did cause a problem for you?

1) A lot of people who have had bariatric surgery tell me that some of their experiences change from before and after surgery. In what ways is this applicable to you?

Probe 1A: What about differences in the way people treated you from before compared to after surgery?

Probe 1B: What about differences in how you view yourself/self-esteem/identity?

Probe 1C: Do you feel you experienced any changes with who you socialized with/spent your free time with after bariatric surgery (i.e. peer group/group of friends?)..

Probe 1D: What about differences in romantic relationships -before compared to after surgery?

Probe 1E: What about your relationship with food...

2) Bariatric surgery patients usually receive education/information about postoperative eating and drinking (alcohol use). Can you tell me what that entailed?

Probe 2a: What did they tell you?

Probe 2b: Were the instructions/information/education written/verbalized??

Probe 2c: Who did you receive the information from (doctor, nurse, or other staff at the bariatric surgery clinic) 
3) I'd like to hear more about your experiences with drinking alcohol before and after surgery..

Probe 3a: What was your drinking like before surgery?

Probe 3b: What about after surgery?

Probe 3c: Did you notice a change (increase/decrease) since your surgery?

Probe 3d: Tell me about drinking right after surgery compared to your current drinking

Probe 3e: Do you feel differently when you drink alcohol now than compared to before surgery?... any changes in alcohol sensitivity?

Probe 3f: Tell me about drinking right after surgery compared to your current drinking

Probe 3 g: Did you ever have any problems with alcohol? (before and after)

Probe 3h: What about challenges controlling your drinking?

1. IF YES-: Please tell me more..

2. Any potential solutions? What worked/helped? What didn't....

Probe 3i: Was alcohol use ever a concern for you...

Probe 3j: How would you know if you developed a problem with drinking in the future? Or what does "problematic drinking” look like to you?

4) Were you trying to avoid alcohol at all after surgery? Can you tell me about that...

Probe 4a: If yes, what challenges did you face with not drinking?

Probe 4b: What was particularly challenging?

Probe 4c: What helped?

Probe 4d: What advice would you give others who are trying to not drink during this period?

5) Is there anything you think would be helpful for others to know about alcohol use after bariatric surgery?

Probe 5a: What about tips on trying to abstain from drinking after surgery.. Probe 5b: What about tips on trying to limit drinking after surgery...

6) For the drinking context scale you just completed you indicated you were most likely to drink excessively (INSERT DRINKING CONTEXTS HERE)

ii. Can you tell more about that?

iii. Do you think it would be difficult to try to not drink in this environment?

iv. What environments do you think would help people abstain from alcohol (if they so desired?) 
7) In the drinking motives questionnaire you indicated you were more likely to drink $\mathrm{X}$. .

v. Can you tell me more about that?

8) Some people have reported that they socialize and/or "go out more" after their surgery. What are your experiences with this?

Probe: If you experienced changes in socialization with and/or how people treated you after you surgery do you think this impacted your alcohol use?

9) Is there anything unique about being an overweight person living in South Florida?

Probe : People also talk about obesity related discrimination/stigmatization. Do you feel you experienced that?

10) Is there anything that I did not ask you about in terms of your experience pre/post bariatric surgery that you think I should know about? 
VITA

\section{CHRISTINE E. SPADOLA}

$1998-2002$

2002-2007

2008

2010 - present

2011- 2015

$2012-2014$

2013

$2014-2015$

2015 - present
B.A., International Studies \& Criminology (double major) University of Miami

Miami, Florida

Research Associate

Center for Drug and Alcohol Studies

University of Delaware (South Florida offices)

Miami, FL

M.S., Psychology

Florida International University

Miami, Florida

Licensed Mental Health Counselor

Florida Department of Health

Research Associate

University of Miami, Miller School of Medicine

Research Fellow

Center for Research on U.S. Latino HIV/AIDS and Drug

Abuse, Florida International University

Interdisciplinary Research Training Institute Fellow

National Hispanic Science Network

Doctoral Candidate

Florida International University

Miami, Florida

Graduate Assistant

Florida International University

\section{PUBLICATIONS AND PRESENTATIONS}

Spadola CE, Wagner EF, Dillon FR, Trepka MJ, de la Cruz-Munoz N, Messiah SE. (2015). Alcohol use among weight loss surgery patients: A review of the literature and future research implications. Alcoholism: Clinical and Experimental Research. DOI: 10.1111/acer.12805. 
Vidot D, Prado G, Spadola C, de la Cruz- Munoz N, Cuesta M, Messiah SE. (2015)PostOperative Marijuana Use and Disordered Eating among Bariatric Surgery Patients. In press, Surgery for Obesity and Related Diseases.

Dillon, F. R., Alessi, E. J., Craig, S. L., Ebersole, R. C.; Kumar, S.M.; \& Spadola, C.E.(2015). Development and Evaluation of the Lesbian. Gay, and Bisexual Counseling Self-efficacy Inventory-Short Form (LGB-CSI-SF).Psychology of Sexual Orientation and Gender Diversity, 2(1), 86-95.

Vidot, D. C., Prado, G., De La Cruz-Munoz, N., Cuesta, M., Spadola, C., \& Messiah, S. E. (2015). Review of family-based approaches to improve postoperative outcomes among bariatric surgery patients. Surgery for Obesity and Related Diseases, 11(2), 451-458.

Spadola, C.E., Wagner E.F., Ruggiano, N., Accornero, V.H., Vidot, D.E., de la CruzMunoz, N.E., Messiah S.E. (2015, April).Urban, young adult bariatric surgery patients: Post-operative alcohol use, depression and anxiety. Abstract accepted for oral presentation at the $45^{\text {th }}$ Annual Urban Affairs Association conference. Miami, FL.

Spadola, C. E., Messiah, S. E., Vidot, D., Dillon, F. R., Wagner, E. F., Moorehead, M., \& De La Cruz-Munoz, N. (2013, October). Post-operative relationship between alcohol use, depression, and anxiety among predominantly Hispanic/Latino young adult bariatric patients. Abstract accepted for oral presentation at the National Hispanic Science Network’s Thirteenth Annual International Conference. Bethesda, MD

Dillon, F. R., Alessi, E. J., Craig, S. L., Ebersole, R. C.; Kumar, S.M.; \& Spadola, C. E.(2015, August). Developing the Lesbian. Gay, and Bisexual Counseling Self-efficacy Inventory-Short Form (LGB-CSI-SF). American Psychological Association 2015 Convention. Toronto, Canada.

Spadola, C.E., Wagner, E.F., Accornero, V.H., Padilla, M, Cuesta, M., de la CruzMunoz, N. Messiah, S.E. (2015, June). Changes in alcohol use behaviors following weight loss surgery among young adults in Miami, FL: A content analysis. $38^{\text {th }}$ Annual Research Society on Alcoholism Scientific Meeting. San Antonio, Texas.

Spadola, C.E., Wagner, E.F., Dillon, F.R., De La Rosa, M., Accornero, V.H., Pulgaron, E., Vidot, D., De La Cruz-Munoz, N., Messiah, S.E. (2014, June). Prevalence of postoperative alcohol use, depression, and anxiety among young adult bariatric surgery patients. $37^{\text {th }}$ Annual Research Society on Alcoholism Scientific Meeting. Bellevue, Washington.

Spadola, C. E., Messiah, S. E., Wagner, E. F, Dillon, F. R., \& Trepka, M. J. (2013, October). Alcohol use among weight loss surgery patients: Current knowledge and future directions. Poster Presentation, National Hispanic Science Network's Thirteenth Annual International Conference. Bethesda, MD. 